\title{
Derivation of the set of the fundamental interactions from first principles
}

\author{
Jiri Voltr ${ }^{\mathrm{a}}(\mathbb{D}$ \\ Stavbaru 205, Strakonice II 38601, Czech Republic
}

Received: 22 July 2021 / Accepted: 10 November 2021

(C) The Author(s) 2021

\begin{abstract}
Global conservation laws require the fundamental interactions to be processes which transfer information from one particle to another. Therefore, in order to show what types of interactions may exist, we derive from the very first principles a set of the most fundamental information transfers and their basic properties. Within these information transfers, we identify candidates for gravitational, electromagnetic and strong scattering, and also for weak decay. We do it by taking the characteristic properties of each fundamental interaction, such as confinement or parity violation, and by using them to rule out information transfers without these properties. The found mapping then makes possible to study the information transfers in order to get knowledge about the corresponding fundamental interactions.
\end{abstract}

\section{Introduction}

One of the unsolved problems in high-energy physics is the question whether all the four known fundamental interactions can be unified into a single theory [1]. The first step toward the answer was made in 1960s when electromagnetic and weak interaction were unified into one electroweak theory [2-5]. The following attempts to make another step have not been successful so far. There have been proposed several models unifying electroweak theory with strong interaction [6-8]. Nevertheless, the viability of these models is substantially restricted by their prediction of proton decay [9], which so far has not been observed [10]. There have been also attempts to include gravity, and nowadays, the most promising models being able to unify all the fundamental interactions are supergravity and superstring models $[11,12]$. All these models, however, lack any experimental evidence as well. The supersymmetry models lack the evidence of the existence of the superpartner particles [13-15], and the string models manifest their properties primarily on the Planck scale, which is far from being testable in today's experiments [16]. The unification of the fundamental interactions, thus, still remains unresolved, and it seems that in order to make progress, we need an entirely novel approach $[17,18]$.

The fundamental interactions transfer information between the interacting particles. It can be seen from the fact that they satisfy various conservation laws. If the interactions did not transfer any kind of information from one particle to another, then the changes on the interacting particles would be mutually uncorrelated, and no global conservation law could ever

\footnotetext{
a e-mail: jiri@voltr.cz (corresponding author)
} 
be systematically satisfied. Consequently, the fundamental interactions do transfer information, i.e., they are information transfers. In this sense, every theory unifying the fundamental interactions must at its core be a theory of information transfers. Since information and its transfers stem from computer science, we use computer science to derive the set of all possible fundamental information transfers. Then, we derive their basic physical properties and look for a relation between the individual information transfers and the individual fundamental interactions. Namely, we take each fundamental interaction, and by ruling out transfers with different properties, we find those transfers which can in principle represent that interaction. The found mapping, then, makes possible to study the properties of the information transfers and, in fact, to get knowledge about the properties of the corresponding fundamental interactions.

Since this interdisciplinary study is intended for readers from the high-energy physics community, the computer science's part is reduced. Nonetheless, it is already clear that it cannot be skipped entirely because computer science serves as the starting point of our model.

\section{Universe versus computer system}

Our Universe contains and processes information. Therefore, it satisfies the broadest definition of a computer system. In agreement with this observation, from now on we assume our Universe is a computer system. The idea that our Universe is a giant computer was originally suggested by Zuse already in 1967 [19], but in recent years the idea has attracted an increasing amount of attention [20-30]. However, it has never been used to address all the fundamental interactions. Note that in the following text we do not require the universal computer system to be based on discrete bits in the way Wheeler's It from bit would require [31].

\subsection{Implementation}

The universal computer system may be implemented in many ways. Nevertheless, all the implementations, once they can produce the same outputs, are equivalent. Thus, it seems that the knowledge of the implementation is of no importance. However, using a concrete implementation and particularly its terminology may help build a firm analogy between the Universe and the computer system. From all the possible implementations, we choose the option that our Universe is implemented as an object-oriented computer system because the object-oriented approach is very intuitive and it has well-established terminology.

Every object-oriented computer system is composed of objects. Each of these objects contains data in the so-called attributes and performs actions by the so-called methods. ${ }^{1}$ These attributes and methods are fully determined by the class of the object. It means that all the objects of the same class have the same attributes and the same methods [32]. At the level of elementary particles, we witness the same thing. Namely, all the particles of the same type have the same properties and under the same circumstances they behave in the same way (they follow the same statistics). Thus, there is an analogy between the elementary particles and the objects of the universal computer system. Concretely, each particle type is a class, and each particle is an object in the system. Each particle, then, has several attributes and several methods which completely describe its properties and behavior, most importantly its interactions.

\footnotetext{
${ }^{1}$ Note that methods can also contain data, but the existence of data within a method is limited to the time period of the method performance.
} 


\subsection{Reference to object}

In object-oriented systems, objects can be linked to other objects. Each link is provided by a reference to an object which has two important properties. Firstly, when object $C$ has a reference to object $D$, let us denote it by $\varepsilon(C, D)$, for object $C$ using the reference is usually the fastest way to change data on object $D$, no matter in what part of memory the object is. Secondly, the system containing objects $C$ and $D$ cannot be treated as a composition of two mutually independent objects, because due to the existence of the reference $\varepsilon(C, D)$, object $C$ can anytime directly influence object $D$, i.e., object $D$ is somehow dependent on object $C$. Quantum physics uses a concept which has both these properties, it is entanglement. When particles $C$ and $D$ are entangled, the entanglement provides the fastest way to influence the other particle, no matter how distant the other particle is. Furthermore, the system of two entangled particles $C$ and $D$ cannot be, by definition, decomposed into mutually independent particles.

These properties allow us to assume that the term reference is equivalent to the term irreducible one-way entanglement. It is irreducible because there is no such thing as part of a reference. It is one-way because every reference has an orientation, i.e., $\varepsilon(C, D)$ does not imply $\varepsilon(D, C)$. If we want to describe the system of entangled particles $C$ and $D$ where a measurement of particle $C$ or $D$ influences particle $D$ or $C$, respectively, then we need two irreducible one-way entanglements, $\varepsilon(C, D)$ and $\varepsilon(D, C)$. In the rest of the article, we talk only about irreducible one-way entanglements, and thus the two adjectives are usually omitted.

\subsection{Identity of objects}

In object-oriented systems, each object has a unique identity. The immediate consequence is that every particle has a unique identity. This seems to be in a direct contradiction with the indistinguishability of particles, which roughly says that all the particles of the same type are indistinguishable because we cannot attach to a particle, say to particle $D$, any mark which would distinguish it from the other particles of the same type. However, entanglement $\varepsilon(C, D)$ is such a mark. It guarantees that whenever particle $C$ uses the entanglement for an action on the entangled particle, the action will be done just on particle $D$ and not on any other particle in the Universe. Without the unique identity of particle $D$ this would be impossible. Moreover, if the entanglement could be used repeatedly, particle $D$ could be distinguished from the other particles of the same type, at least in principle. We conclude that the indistinguishability of particles, rather than being an intrinsic property of the system, is an emergent property. It emerges when particles are not entangled or when particles are entangled in the very same way. In total, before an application of the indistinguishability of particles we always must take into consideration the fact whether and how particles are entangled.

The unique identity of an object is in computer systems represented by a unique identifier. Since every identifier is a piece of data, it can be stored. For example, the identifier of object $D$ stored in an attribute of object $C$ is, in fact, entanglement $\varepsilon(C, D)$, which allows object $C$ to do some actions on object $D$. For the creation of $\varepsilon(C, D)$ is, therefore, necessary the knowledge of the identifier of object $D$. The question is where its value comes from. The value of the identifier of object $D$ is determined at the moment of the creation of object $D$ and originally resides only in the object itself in what we call self-identifier. Thus, entanglement $\varepsilon(C, D)$ can be created only when the identifier of object $D$ is through a series of actions delivered from the self-identifier of object $D$ to an attribute of object $C$. Note that the self- 
identifier of object $D$, which can be symbolically written as $\varepsilon(D, D)$, is an implicit property of the object with no way to change its value. The constancy of the value of the self-identifier of object $D$ then implies that all the entanglements that point to object $D$ are usable during the entire life span of object $D$.

\subsection{Actions on objects}

Objects can do various actions on objects they have access to. Namely, object $C$ with complete access to particle $D$ can access an attribute of object $D$ to take away some data or to put there some data; it can also read the self-identifier of object $D$ to get to know its identity; it can read an entanglement stored in object $D$, that is to get the identifier of object $E$ if there is $\varepsilon(D, E)$; it can also rewrite the entanglement stored in object $D$ to a new value, that is to replace $\varepsilon(D, E)$ with entanglement $\varepsilon(D, F)$, provided object $C$ knows the identifier of object $F$. Finally, object $C$ can call a method on object $D$ so object $D$ may do some actions for object $C$. Any of these actions can be done by object $C$ on object $D$ if the former object has complete access to the latter object. There are three basic cases when it can happen. Firstly, when $C=D$, that is object $C$ has access to itself. Secondly, when object $C$ has access to object $D$ via entanglement $\varepsilon(C, D)$, and finally, a physically interesting case when objects $C$ and $D$ are co-local. These three basic ways of access to an object can, of course, be combined.

\subsection{Optimal system}

From the computer science's point of view, it is important to ask the question whether a computer system is optimal. When we talk about an optimal system, we usually mean that at least one of the following metrics is minimal:

(a) time complexity, i.e., how many elementary operations the system must do in order to accomplish every task,

(b) space complexity, i.e., how much of the memory the system needs to allocate in order to accomplish every task.

Our Universe can do a vast amount of actions simultaneously. In such a parallelly working system, time complexity is of no or a very low importance. To the universal computer system can, therefore, be relevant only space complexity. The term space complexity, however, may cause some confusion because it has nothing to do with space in the sense of the stage for physical processes. For that reason, we rename it to memory complexity. There are two kinds of optimal systems in the sense of memory complexity: actively optimal systems and passively optimal systems. By the actively optimal system, we mean that the computer system decreases the memory complexity whenever there is an opportunity to do that, and by the passively optimal system we mean that the system chooses an optimal starting point of objects, and since then it does not address the optimization. We choose the option that our Universe is a passively optimal system. ${ }^{2}$ Thus, for the universal computer system we postulate:

Postulate 1 (Optimal system) The universal computer system is passively and locally optimal in the sense of memory complexity.

The universal computer system is locally optimal when every of its objects is optimal. It means that the minimal memory complexity is inbuilt in the attributes and methods of the objects. The object attributes permanently occupy memory, and thus they always increase the memory

2 We are motivated by the (so far incomprehensible) fact that in passively optimal systems weak decays have always three products and in actively optimal systems weak decays have only two products. 
complexity. The immediate consequence is that the usage of the attributes is minimized. Most properties of every particle are, therefore, implicitly included in the methods of the object rather than being explicitly stored in the attributes of that object. It implies an important characteristic of the universal computer system. Namely, that our Universe prefers action over data. A consequence is that if the system needs to use a value, then whenever possible, it evaluates the present value rather than it uses a historical value stored in an attribute. This can be rephrased in the way that whenever possible, there is no concrete value until it is needed.

\section{Physical description of processes}

Building a physical model requires a way to describe processes and their events. Hence, in this section we define the stage for physical processes and specify the description of the state of the physical system we will use.

\subsection{Physical stage}

From the physical point of view, it is important to have a stage for physical processes. It means to have time and space which can be used to describe all possible processes and their events. Here we are interested in time and space (hereafter called fundamental) within which the universal computer operates. We have a simple but very strict criterion for the fundamental time and space: fundamental time must provide a universally valid unique order of all the events and fundamental space must provide universally valid unique relations among the locations of all the events. This makes possible to describe each process (such as a correlation via an entanglement) by a unique sequence of unique consecutive steps that the universal computer performs. Moreover, as we will see, the uniqueness of the order of the events makes possible a natural classification of processes, that is a natural classification of the fundamental interactions.

Relative time and space cannot be the fundamental time and space. Firstly, it is because relative time and space cannot be used to describe all possible events, namely they cannot be used within the reference frame of a photon and within a reference frame inside a black hole. Secondly, it is because two observers may within relative time disagree on the order of distant events. That is, relativity of simultaneity ruins the uniqueness of the order of the events. On the other hand, relative time and space are absolutely inevitable for describing the local processes in terms of what we are going to measure. Consequently, we need two notions of time and space. Since fundamental time and space are expected to be unique, relative time and space should be derivable from the fundamental ones. The existence of two notions of time and space may seem to be problematic [33]; however, it is not because they do not mix in their roles. The fundamental time and space are not locally measurable, and relative time and space do not serve as the operational base for the universal computer system. Moreover, since the relative time and space are derivable from the fundamental ones, in terms of superiority there is a clear relation between them.

Ruling out relative time and space indicates that in our search for the fundamental time and space we should abandon local quantities. This leads us to the fact that an observation of a distant event is not one and the same thing as the event itself. For example, we can say that although we observe the explosion of a supernova here and now, it occurred at a large distance several million years ago. Thus, besides the time and location of the observation of the event, we can also think about the time and location of the occurrence of the event. Using the time and location of the occurrence of events has a disadvantage that every result must 
be before a comparison with a local observation transformed to the observational time and space. On the other hand, the main advantage is that the time order of the occurrence of all the events can be unique. Since this is exactly what we need, we use time and location of the occurrence of events as a synonym for the fundamental time and space.

Postulate 1 dictates to minimize the number of local parameters which completely describe the geometry of the fundamental time and space. Therefore, the geometry of fundamental time and space should be described by global parameters rather than local ones. It means they should be location-independent, i.e., homogeneous, and direction-independent, i.e., isotropic. According to these properties, we choose that the fundamental time and space are euclidean. Moreover, since we require unique ordering within fundamental time, time is a one-dimensional oriented quantity. This leads us to the second postulate:

Postulate 2 (Time and space) There exists one-dimensional euclidean fundamental time which flows only in one direction, and d-dimensional euclidean fundamental space.

The fundamental time and space are the time and space within which the universal computer system operates. Nevertheless, in their definition still remain ambiguities in the scale of space and the pace of time. Since these ambiguities can be resolved only in terms of constants of Nature which we still do not have, we leave them unresolved and use the fundamental time and space in this article anyway.

\subsection{Perspective and retrospective description}

There are two ways to describe the state of a physical system. The first one, a perspective description, is what we usually mean by quantum description. The state of the system is there described by potential values of the measurement outcomes and their amplitudes. Once we know the initial state and the time evolution of the system, we can, in principle, calculate the state of the system for any later time, provided no measurement has occurred. The advantage of the perspective description is, therefore, that it allows us to make quantitative probabilitybased predictions. Nevertheless, there is also a disadvantage. The perspective description can in no way say the moment when a measurement will occur and what exact outcomes the measurement will produce. There is a complementary way to describe the same system which can do that. It is a retrospective description. In the retrospective description, the state of the system is described by concrete values obtained by measurements at concrete moments. In other words, the retrospective description can for a small number of moments say the exact outcomes. On the other hand, the disadvantage is that a single retrospective description cannot be used to make any quantitative predictions. Only a sufficiently large set of retrospective descriptions may reveal the underlying probabilities and make possible to create the perspective description.

None of these descriptions is superior to the other. Each of them can describe something the other cannot, and thus each of them has its own usage and importance. In this article, we use the retrospective description within which the value of information $q$ contained in object $C$ at time $t$ will be denoted by $q_{C}(t)$. We focus on lossless information $q$, or equivalently on conserved quantities, because in a closed system the corresponding conservation laws may enhance our knowledge of the state of the system from few discrete moments to continuous time intervals. 


\section{Information transfers}

One of the most fundamental operations in the computer system is the transfer of information from one object to another. In this article, we study only fundamental information transfers. By a fundamental information transfer, we mean it satisfies the following definition:

Definition 1 (Fundamental information transfer) An information transfer between two distant objects $A$ and $B$ is called fundamental, if and only if

(a) Objects $A$ and $B$ and mediator $M$ are the only participants in the information transfer.

(b) The information transfer is initiated by creating mediator $M$ and handing control over the transfer immediately to the mediator.

(c) During the entire information transfer, there are two point information changes on objects $A$ and $B$, namely one on object $A$ and one on object $B$.

(d) Changes of information on mediator $M$ correlate in time and value with the changes on objects $A$ and $B$.

(e) Mediator $M$ annihilates itself at the end of the information transfer.

(f) There are no other events in the transfer.

Each of objects $A$ and $B$ is meant to be a single particle. On the other hand, for the reasons that will be revealed later we do not require mediator $M$ to be a structureless single particle.

\subsection{Timing of information transfers}

Timing is a key property of any process. Since due to postulate 2 time flows only in one direction, we can talk about the beginning of the transfer and about the end of the transfer. The time of the beginning of the transfer, i.e., the time when mediator $M$ is created, will be denoted by $\tau_{0}$. Similarly, the time of the end of the transfer, i.e., the time when the mediator is annihilated, will be denoted by $\tau_{E}$. Finally, since the changes on objects $A$ and $B$ are point changes, we can assign to each of them a specific time. Let $\tau_{A}$ and $\tau_{B}$ be the times of the change of information on object $A$ and on object $B$, respectively.

A naïve picture of a transfer of conserved quantities from particle $A$ to a distant particle $B$ requires

$$
\tau_{0}=\tau_{A}<\tau_{B}=\tau_{E}
$$

In that case, the mediator is created at the same time as the change of conserved quantities on particle $A$ occurs, and the mediator is annihilated at the same time as the change of conserved quantities on particle $B$ occurs. However, this is not the only way the conserved quantities can be transferred from particle $A$ to particle $B$. In computer science, the transferred information is not necessarily attached to mediator $M$ so tighly as in the case of the naïve picture of the transfer. Firstly, information changes on objects $A$ and $B$ (made at time $\tau_{A}$ and $\tau_{B}$, respectively) must be made within the existence of the mediator, i.e., within $\left[\tau_{0}, \tau_{E}\right]$. Moreover, once we mark object $A$ as the object from which the information goes, i.e., $\tau_{A} \leq \tau_{B}$, there is no requirement for $\tau_{A}<\tau_{B}$. It leads to an inequality which becomes our third postulate:

Postulate 3 (Timing) The timing in any transfer of information from object A to object $B$ satisfies

$$
\tau_{0} \leq \tau_{A} \leq \tau_{B} \leq \tau_{E}
$$


Time inequality (2) contains three symbols $\leq$, and each of them represents two options, = and $<$. In other words, postulate 3 does not provide one timing, but eight possible timings of information transfers.

\subsection{Role distribution in information transfers}

The participants of an information transfer play various roles. We can talk about the initiator of the transfer, that is the object which creates mediator $M$ and immediately hands control over the transfer to the mediator. It will be denoted by $R$. We can also talk about the location of the mediator at the beginning of the transfer and its location at the end of the transfer. Let objects $S$ and $T$ be the objects where the mediator starts and terminates, respectively.

A naïve picture of a transfer of conserved quantities from particle $A$ to particle $B$ requires

$$
R=A, \quad S=A, \quad T=B,
$$

while computer science is less restrictive and requires:

Postulate 4 (Role distribution) The role distribution in any transfer of information from object A to object B satisfies

$$
R \in\{A, B\}, \quad S \in\{A, B\}, \quad T \in\{A, B\} .
$$

Each of objects $R, S$, and $T$ can be either object $A$ or object $B$. It means that postulate 4 allows eight possible role distributions.

\subsection{List of information transfers}

The timings and role distributions can be combined to produce the list of information transfers. Since they are mutually independent, there are 64 different ways to transfer information from one object to another. The naming convention of the information transfers and their mediators is as follows ${ }^{3}$ : Let $i \in \mathbb{Z}_{64}$ be expressed in base- 2 numeral system as

$$
(i)_{10}=(\operatorname{ls} r o a b)_{2} \text {, }
$$

where $l, s, r, o, a$, and $b$ are binary digits. If for an object $X \in\{A, B\}$, we define $\bar{X}$ as the complementary object to object $X$ within set $\{A, B\}$, that is

$$
\bar{X}=\{A, B\} \backslash X,
$$

then $M_{i}$ represents the mediator of information transfer $I_{i}$ that satisfies:

(a) $T=\bar{S}$ when $l=0$, while $T=S$ when $l=1$ (note that $l$ stands for loop),

(b) $S=A$ when $s=0$, while $S=B$ when $s=1$,

(c) $R=A$ when $r=0$, while $R=B$ when $r=1$,

(d) $\tau_{0}=\tau_{A}$ when $o=0$, while $\tau_{0}<\tau_{A}$ when $o=1$,

(e) $\tau_{A}=\tau_{B}$ when $a=0$, while $\tau_{A}<\tau_{B}$ when $a=1$,

(f) $\tau_{B}=\tau_{E}$ when $b=0$, while $\tau_{B}<\tau_{E}$ when $b=1$.

For example, since $(21)_{10}=(010101)_{2}$, information transfer $I_{21}$ satisfies $T=A, S=B$, $R=A$, and finally $\tau_{0}<\tau_{A}=\tau_{B}<\tau_{E}$.

3 Those who attended our lectures in 2017 and 2018 will notice that we no longer use the original naming convention of the information transfers based on how we gradually developed the model. For the first time, we use a naming convention based on its practicality. 


\subsection{Scope of study}

In this article, we do not study all the fundamental information transfers. We focus on those transfers which take a non-zero time,

$$
\tau_{0}<\tau_{E}
$$

and do not contain any loop,

$$
T=\bar{S} \text {. }
$$

Postulate 3 allows eight distinct timings of information transfers. Nevertheless, scope condition (7) dictates the exclusion of the transfers with $\tau_{0}=\tau_{A}=\tau_{B}=\tau_{E}$. Thus, here we study only seven timings of information transfers. Similarly, postulate 4 allows eight possible role distributions. However, we study only those transfers, whose particle $T$ is determined by equation (8). It means we study only four ways of role distributions. In total, we study 28 types of non-instantaneous information transfers without any loop. The set of their indexes is as follows:

$$
\mathbb{F}=\mathbb{Z}_{32} \backslash\{0,8,16,24\}
$$

Although the number of the studied transfers is still quite large, the grouping of transfers with similar properties will significantly simplify the overall picture of the information transfers.

\section{Lossless information}

All the information transfers require splitting information into two pieces and combining two pieces of information into one. The conservation law of lossless information $q$ for both operations can be expressed by a single symbolic operator $\oplus_{q}$. If $t_{1}<t_{2}$, then

$$
q_{C}\left(t_{1}\right)=q_{D}\left(t_{2}\right) \oplus_{q} q_{E}\left(t_{2}\right)
$$

describes the splitting of information $q$ in process $C \rightarrow D+E$. If $t_{1}>t_{2}$, then equation (10) describes the combining of information $q$ in process $D+E \rightarrow C$. The operator $\oplus_{q}$ is a binary operator over the set of all possible values of $q$, $\operatorname{dom}(q)$. If $\operatorname{dom}(q)$ contains a neutral element, then it is denoted by $q_{0}$. The subscript $q$ in $\oplus_{q}$ indicates that different types of information may use different operators for the summation. Whenever the subscript is obvious from the information present in the summation, it is omitted.

Equation (10) does not describe all possible processes and should be generalized. The generalization is straightforward and the conservation law of $q$ in process $C_{1}+\ldots+C_{n} \rightarrow$ $D_{1}+\ldots+D_{m}$ can be written as

$$
\bigoplus_{i=1}^{n} q_{C_{i}}\left(t_{1}\right)=\bigoplus_{j=1}^{m} q_{D_{j}}\left(t_{2}\right),
$$

where $t_{1}<t_{2}$. The problem is that we do not know whether the operation $\oplus$ is commutative and/or associative. It means that we do not know the order in which we must sum the individual values of $q$. In the case of commutative and associative operation $\oplus$, the order of the summation is irrelevant, we always get the same result. 


\subsection{Visualization of information transfers}

Each information transfer can be visually presented in a diagram which clearly shows the changes of conserved quantities. Such a diagram will be called $q$-diagram, where $q$ in the name stands for any transferable conserved quantity. In a $q$-diagram time flows either from the bottom of the diagram to the top, or from the left side of the diagram to the right side. We use the former option. Particles $A$ and $B$ are presented by a solid line and mediator $M$ is presented by a dashed line. Whenever the lines of two participants touch each other, we interpret it in the way that the participants are co-local. On the other hand, if the lines do not touch each other, we interpret it in the way that the participants are not co-local, or at least they behave as if they were not co-local (i.e., they ignore each other). A full dot in a $q$-diagram indicates the event of the initiation of the information transfer on particle $R$ at time $\tau_{0}$. No or a smooth continuous change of direction of a particle (or a mediator) means no change of information $q$ on the particle (or the mediator). If possible, we use no change of direction in that case. On the other hand, a sharp instant change of the direction of a particle (or a mediator) means a change of information $q$ on the particle (or the mediator), or at least the possibility of such a change. Definition 1 guarantees only one change of information on object $A$ made at time $\tau_{A}$, and only one change of information on object $B$ made at time $\tau_{B}$. The conservation law of $q$, which is satisfied at every moment, guarantees that a change of information on one object made at some time is accompanied by a corresponding change of information on another object made at the same time. Since in each transfer there are no other participants than $A, B$ and $M$, changes of information on mediator $M$ can only be made at times $\tau_{A}$ and $\tau_{B}$. The $q$-diagrams of information transfers $I_{0}$ to $I_{31}$ are presented in Table 1 and in Table 2.

\subsection{Information content}

In the information transfers, we are interested in the information that is contained in its participants. All the events of each transfer are at times $\tau_{0}, \tau_{A}, \tau_{B}$ and $\tau_{E}$, and thus we will need to work with time phases bounded by these times. Let us use the following convention: time $\tau_{X-}$ stands for any time satisfying $\tau_{X-}<\tau_{X}$ where $X \in\{0, A, B, E\}$, and time $\tau_{X+}$ stands for any time satisfying $\tau_{X+}>\tau_{X}$ where $X \in\{0, A, B, E\}$. Finally, $\tau_{X Y}$ stands for any time satisfying $\tau_{X}<\tau_{X Y}<\tau_{Y}$ where $X, Y \in\{0, A, B, E\}$ and $X \neq Y$.

Let us start by looking for the information that is contained in mediator $M$. The only information change on particle $A$ occurs at time $\tau_{A}$ and the only information change on particle $B$ occurs at time $\tau_{B}$. It can be rephrased: The transferred information, hereafter denoted by $\triangle q$, leaves particle $A$ at time $\tau_{A}$ and arrives onto particle $B$ at time $\tau_{B}$. It means that mediator $M$ contains $\triangle q$ only in time interval $\left(\tau_{A}, \tau_{B}\right)$, that is

$$
q_{M}\left(\tau_{A B}\right)=\Delta q
$$

For the information transfers with $\tau_{A}=\tau_{B}$, it means that information $\Delta q$ is not stored in the mediator and is transferred from particle $A$ directly to particle $B$.

At time $\tau_{0}$ mediator $M$ is created on particle $S$ in the process $S \rightarrow S+M$. For the information transfers with $\tau_{0}<\tau_{A}$, the conservation law of $q$ in the creation process requires

$$
\begin{aligned}
& q_{S}\left(\tau_{0-}\right)=q_{S}\left(\tau_{0 A}\right) \oplus q_{M}\left(\tau_{0 A}\right), \text { or } \\
& q_{S}\left(\tau_{0-}\right)=q_{M}\left(\tau_{0 A}\right) \oplus q_{S}\left(\tau_{0 A}\right) .
\end{aligned}
$$




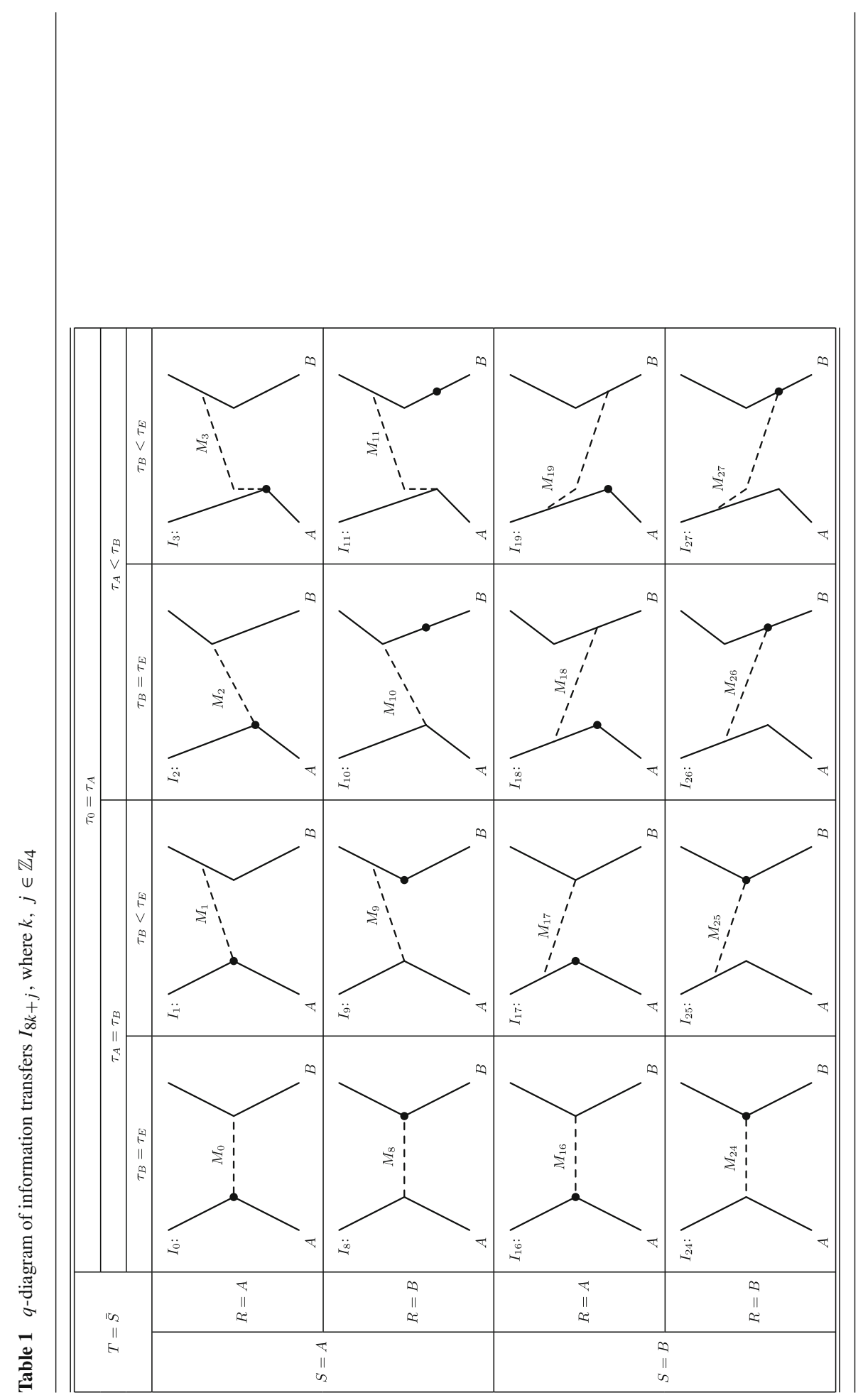


Since there is no change of information $q$ on particle $S$ between times $\tau_{0-}$ and $\tau_{0 A}$, it is $q_{S}\left(\tau_{0-}\right)=q_{S}\left(\tau_{0 A}\right)$. By using it in equations (13a) and (13b), we get that the value $q_{M}\left(\tau_{0 A}\right)$ plays the role of a right or left neutral element of $\operatorname{dom}(q)$, that is

$$
q_{M}\left(\tau_{0 A}\right)=q_{0} .
$$

Similarly, at time $\tau_{E}$ mediator $M$ is anihilated on particle $T$ in the process $T+M \rightarrow T$. For the information transfers with $\tau_{B}<\tau_{E}$, the conservation law of $q$ in the anihilation process requires

$$
\begin{aligned}
& q_{T}\left(\tau_{B E}\right) \oplus q_{M}\left(\tau_{B E}\right)=q_{T}\left(\tau_{E+}\right), \text { or } \\
& q_{M}\left(\tau_{B E}\right) \oplus q_{T}\left(\tau_{B E}\right)=q_{T}\left(\tau_{E+}\right) .
\end{aligned}
$$

Since there is no change of information $q$ on particle $T$ between times $\tau_{B E}$ and $\tau_{E+}$, it is $q_{T}\left(\tau_{B E}\right)=q_{T}\left(\tau_{E+}\right)$. By using it in equations (15a) and (15b), we get that the value $q_{M}\left(\tau_{B E}\right)$ plays the role of a right or left neutral element of $\operatorname{dom}(q)$, that is

$$
q_{M}\left(\tau_{B E}\right)=q_{0} .
$$

Equations (14) and (16) imply that mediator $M$ does not necessarily contain $\triangle q$ during its entire existence. Thus, we must distinguish the information $q$ contained in the mediator at a specific time from the information $\Delta q$ transferred by the mediator.

The remaining task is to find out what information is contained in particles $A$ and $B$. Let us start with the transfers satisfying $\tau_{A}<\tau_{B}$. Since we do not know whether operation $\oplus_{q}$ is commutative, there are two ways to express the relation between $q_{A}\left(\tau_{0-}\right)$ entering the information transfer and $q_{A}\left(\tau_{E+}\right)$ leaving the transfer,

$$
\begin{aligned}
& q_{A}\left(\tau_{0-}\right)=q_{A}\left(\tau_{E+}\right) \oplus \triangle q, \text { or } \\
& q_{A}\left(\tau_{0-}\right)=\Delta q \oplus q_{A}\left(\tau_{E+}\right) .
\end{aligned}
$$

Similarly, there are two ways to express the relation between entering $q_{B}\left(\tau_{0-}\right)$ and leaving $q_{B}\left(\tau_{E+}\right)$,

$$
\begin{aligned}
& q_{B}\left(\tau_{E+}\right)=\Delta q \oplus q_{B}\left(\tau_{0-}\right), \text { or } \\
& q_{B}\left(\tau_{E+}\right)=q_{B}\left(\tau_{0-}\right) \oplus \Delta q .
\end{aligned}
$$

An example of the information content of the participants of an information transfer with $\tau_{A}<\tau_{B}$ is in Fig. 1 .

In the transfers with $\tau_{A}=\tau_{B}$ once we fix the order of summation for $\tau_{0-}$, we can only write

$$
\begin{aligned}
& q_{A}\left(\tau_{0-}\right) \oplus q_{B}\left(\tau_{0-}\right)=q_{A}\left(\tau_{E+}\right) \oplus q_{B}\left(\tau_{E+}\right), \text { or } \\
& q_{A}\left(\tau_{0-}\right) \oplus q_{B}\left(\tau_{0-}\right)=q_{B}\left(\tau_{E+}\right) \oplus q_{A}\left(\tau_{E+}\right),
\end{aligned}
$$

because there is no participant which would hold $\Delta q$ for a non-zero time and could appear in the conservation law of $q$. Nevertheless, we will still talk about taking $\Delta q$ from particle $A$ and putting $\triangle q$ to particle $B$ in this case.

\subsection{Time of determination of $\triangle q$}

The determination of $\Delta q$ influences the behavior of mediators. Let us examine the time of that event. In each information transfer, there are two moments when information is changed, one at time $\tau_{A}$ and the other at time $\tau_{B}$. Due to the conservation law of $q$, the same value $\triangle q$ 


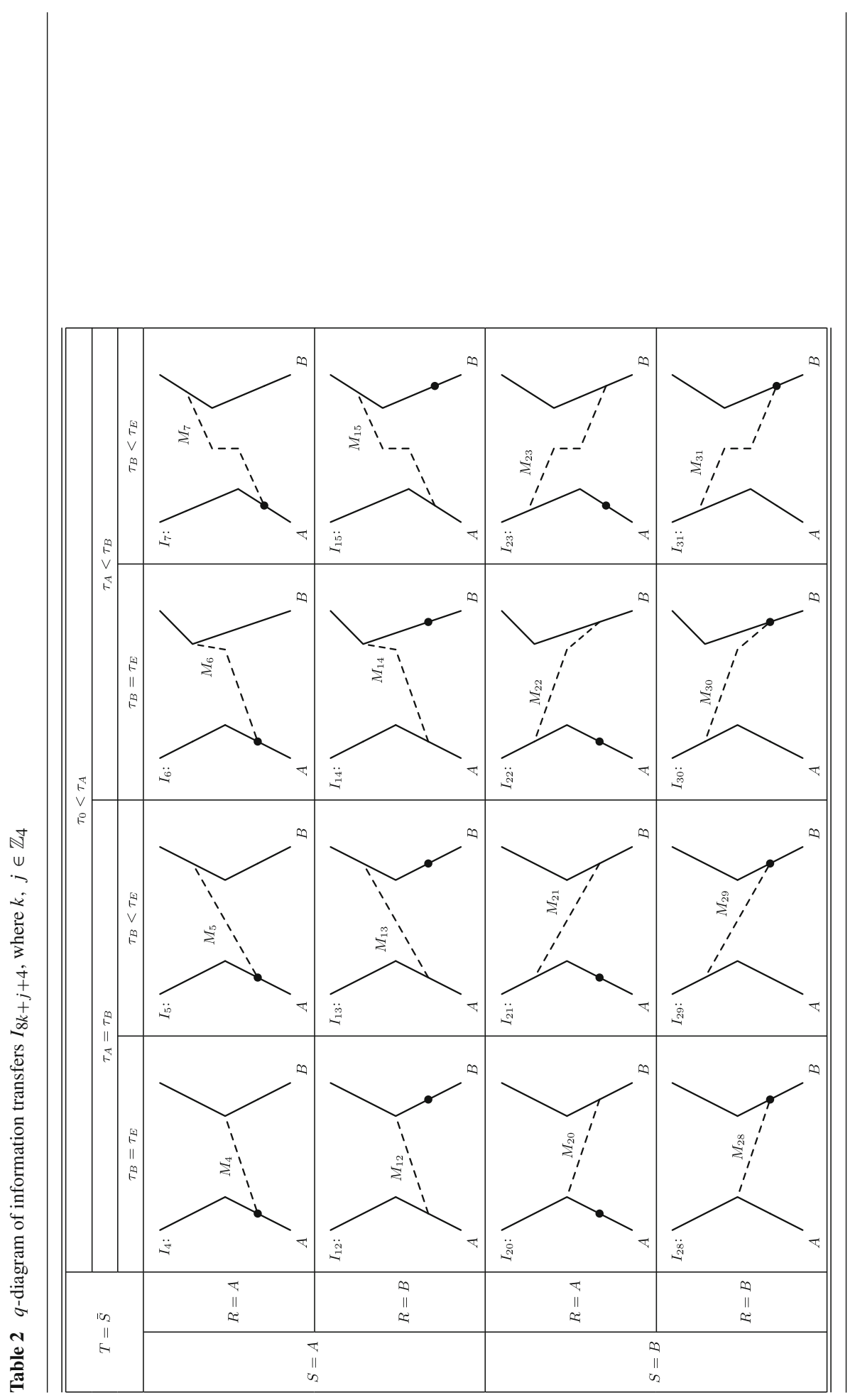




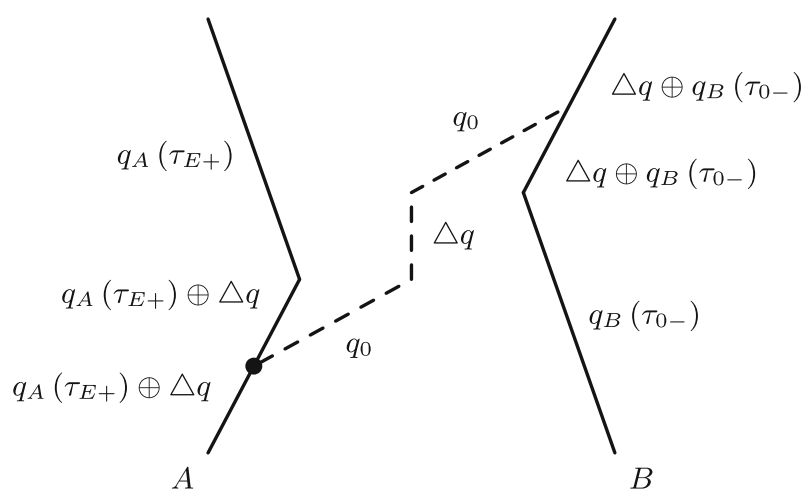

Fig. 1 Information content of the participants of transfer $I_{7}$, a transfer satisfying $\tau_{0}<\tau_{A}<\tau_{B}<\tau_{E}$, and thus having all the possible time phases. It is assumed equations (17a) and (18a) are satisfied

is used within both changes. Consequently, the changes must be causally connected. Since time can flow only in one direction, postulate 2 dictates that $\triangle q$ must be known at the first moment the value is used. Since $\tau_{A} \leq \tau_{B}$, the value of $\Delta q$ must be determined at time $\tau_{A}$ or earlier. If $\triangle q$ were determined earlier than at time $\tau_{A}$, then the value should be remembered, i.e., stored in the system until $\tau_{A}$ when it is used for the first time. On the other hand, if $\triangle q$ is determined just at time $\tau_{A}$, it can be immediately used and no extra storage of the value is needed. The minimum memory complexity in postulate 1 prefers the latter way, that is $\triangle q$ is always determined at time $\tau_{A}$.

\subsection{Quantum measurement?}

The value of information $\Delta q$, which will be transferred within a transfer, may be unknown at the beginning of that transfer. Namely, if a transfer satisfies $\tau_{0}<\tau_{A}$, then the value of $\triangle q$ is not determined at the beginning of the transfer at $\tau_{0}$, and is determined later at time $\tau_{A}$. This is what we expect in quantum systems: at the beginning of the transfer, a system is described by a general state vector usually not being an eigenvector of operator $\hat{q}$, and thus $\Delta q$ has no any specific value. Then, sometime later occurs a quantum measurement, where the state vector becomes an eigenvector of $\hat{q}$, and $\Delta q$ gets a single value equal to the corresponding eigenvalue. The determination of $\Delta q$ could be, therefore, interpreted as a quantum measurement. However, since we are the level of a single performance of an information transfer, that is at a substantially lower level than the level where any quantum measurement is expected to operate, we call it submeasurement. The future research should tell us what relation between a submeasurement of $\triangle q$ and a quantum measurement of $\triangle q$ is. 4

There are three moments when the submeasurement of the value $\triangle q$ can with respect to the lifetime of a transfer occur. In the transfers with $\tau_{0}<\tau_{A}=\tau_{E}$, the submeasurement of $\Delta q$ occurs at the end of each transfer. In the transfers with $\tau_{0}<\tau_{A}<\tau_{E}$, the submeasurement of $\Delta q$ occurs at a moment in between the beginning and the end of each transfer. Finally, in the transfers with $\tau_{0}=\tau_{A}$ the submeasurement of $\Delta q$ occurs right at the beginning of each transfer. Moreover, since due to the conservation law of $q$ the value of $\Delta q$ is not changed at any later time, the transfers with $\tau_{0}=\tau_{A}$ have the value of $\Delta q$ fixed during their entire lifetime.

4 We expect something like the following: A measurement is a process which causes that all the submeasurements have in some sense the same outcome. 
They behave in a very classical way. Besides that, since the submeasurement of $\Delta q$ occurs right at the beginning of a transfer which itself is initiated spontaneously, it seems that there is no way to force that submeasurement, i.e., the submeasurement of $\Delta q$ is done automatically. This leads us to the fact that we should distinguish two types of submeasurement of $\Delta q$ : outer submeasurement and inner submeasurement. By the outer submeasurement, we mean that an intervention forced the universal computer system to determine a concrete value of a physical quantity, and without that intervention no value would be determined. Note that the intervention is what common measurement devices do. On the other hand, by the inner submeasurement we mean the system determines a value of the physical quantity by itself. No intervention is needed for an inner submeasurement, and more strictly, no intervention can cause an inner submeasurement. The transfers with $\tau_{0}=\tau_{A}$ definitely perform an inner submeasurement of $\triangle q$.

\subsection{Measurability of information content}

We distinguish two types of mediators depending on their information content $q_{M}$. If at some moment $t$ mediator $M$ contains information $q_{0}$, i.e., $q_{M}(t)=q_{0}$, we call it virtual. On the other hand, if the mediator contains information $\Delta q$, i.e., $q_{M}(t)=\Delta q$, we call it real. Let us ask whether we can measure the information content of a real mediator, that is to measure $\triangle q$. Information $q$ on particle $A$ is at time $\tau_{A}$ changed by value $\Delta q$. Similarly, information $q$ in particle $B$ is at time $\tau_{B}$ also changed by value $\triangle q$. Therefore, in principle, we can measure any of these changes to get $\Delta q$ which is the information content of the real mediator.

Now, let us ask whether we can measure the information content of a virtual mediator. Measuring the information content of virtual mediator $M$ directly by particle $C \notin\{A, B\}$ is not possible because definition 1 guarantees that in the transfer there are no participants other than $A, B$, and $M$. Therefore, the only way to measure the information content of the mediator is on particle $A$ and/or on particle $B$ via the changes of their information content. There are four possible moments when we can do it, at time $\tau_{0}, \tau_{A}, \tau_{B}$ and at time $\tau_{E}$. Measuring the change of information $q$ on particle $A$ at time $\tau_{A}$ and measuring the change of information $q$ on particle $B$ at time $\tau_{B}$ are not helpful. This is because information $q$ on particles $A$ and $B$ are changed by value $\triangle q$, which does not say anything about the information content of the mediator, which is $q_{0}$. The remaining moments are $\tau_{0}$ and $\tau_{E}$. In the transfers with $\tau_{0}<\tau_{A}$ the information $q$ on particle $S$ is at time $\tau_{0}$ changed by value $q_{0}$. However, $q_{0}$ is a neutral element. It means that $q_{S}$ is not changed at all. Therefore, from the experimental point of view, we cannot distinguish the situation where mediator $M$ left particle $S$ from a situation where no information transfer was initiated. In both cases, $q_{S}$ is not changed. The argumentation is valid also for measuring the change of $q$ on particle $T$ at time $\tau_{E}$ within the transfers with $\tau_{B}<\tau_{E}$. We conclude that the information content of virtual mediators is not measurable.

\section{Entanglements}

Information can be transferred in a contact way or in a non-contact way. For example, in information transfer $I_{2}$ information $\Delta q$ is at time $\tau_{A}$ instantly transferred from particle $A$ to co-local mediator $M_{2}$, that is $\Delta q$ is transferred in a contact way. On the other hand, in information transfer $I_{7}$ (see Fig. 1) $\Delta q$ must at time $\tau_{A}$ be instantly transferred from particle 
$A$ to a distant mediator $M_{7}$. Such a non-local ${ }^{5}$ correlation of conserved quantity $q$ cannot be performed in any contact way. The only known solution is that $\Delta q$ is sent via an entanglement between $A$ and $M_{7}$. Here we conclude that some information transfers require entanglements in order to work.

Every irreducible entanglement is a piece of data stored in the memory of the universal computer system. Therefore, it should be the subject of postulate 1 . The minimum memory complexity of the system can only be reached when every elementary object $C$ has at every moment maximally one self-identifier and maximally $N_{\varepsilon}^{\max }$ outgoing entanglements, $N_{\varepsilon}^{\max }$ being a relatively small number. In terms of information transfers, the minimum memory complexity can be reached only when the number of distinct irreducible one-way entanglements used in each information transfer is minimal. Therefore, in this section we look within each information transfer for the minimum set of entanglements which allows the transfer to do all its actions. Namely, to do the four following actions: particle $R$ at time $\tau_{0}$ creates mediator $M$ on particle $S$, mediator $M$ at time $\tau_{A}$ takes $\triangle q$ from particle $A$, mediator $M$ at time $\tau_{B}$ puts $\triangle q$ into particle $B$, and mediator $M$ at time $\tau_{E}$ hits particle $T$. However, before we start to do the entanglement analysis, we need some preparation.

\subsection{Knowledge of target particle $T$}

In most information transfers, the identity of target particle $T$ is known before mediator $M$ hits particle $T$ at time $\tau_{E}$. It is caused by the definition 1 which requires particles $A$ and $B$ and mediator $M$ to be the only participants of the transfer and the fact that particles $A$ and $B$ play in the transfer various roles at various times. Namely, if each of particles $A$ and $B$ plays some role before the end of the transfer, then we can use equation (8) to determine the identity of target particle $T$ from the knowledge of what transfer $I_{i}$ we are studying.

The role of particle $R$ and particle $S$ are being played at time $\tau_{0}$. Therefore, in the transfers with $\tau_{0}<\tau_{E}$ if $\{R, S\}=\{A, B\}$, then the identity of particle $T$ can be determined from equation (8) at time $\tau_{0}$, that is before the mediator hits the particle. The condition is satisfied when $R \neq S$. Similarly, the role of particle $A$ is being played at time $\tau_{A}$. Thus, for the transfers with $\tau_{A}<\tau_{E}$, if $\{R, S, A\}=\{A, B\}$, then we can determine the identity of particle $T$ at time $\tau_{A}$. The condition is satisfied if $R=B$ or $S=B$. Finally, the role of particle $B$ is being played at time $\tau_{B}$. Consequently, in the transfers with $\tau_{B}<\tau_{E}$, if $\{R, S, A, B\}=\{A, B\}$, then the identity of particle $T$ is known at time $\tau_{B}$. This condition is satisfied always. The three results can be summarized as follows: Information transfers $I_{2}, I_{4}, I_{6}$ and $I_{28}$ are the only transfers in which the identity of target particle $T$ can be unknown until time $\tau_{E}$, the time when mediator $M$ hits the particle. On the other hand, within the other information transfers mediator $M$ looks for the specific particle $T$ to hit it and finish the transfer.

\subsection{Two entanglements are sufficient}

All the information transfers can work with only two entanglements. Let us assume there exist entanglements $\varepsilon(R, \bar{R})$ and $\varepsilon(M, R)$. The initiator of the transfer, particle $R$, does only one thing: it creates mediator $M$ on particle $S$. If $R=S$, then particle $R$ does not need any entanglement to do that because it creates the mediator on itself. On the other hand, if $R \neq S$, then particle $R$ in order to create mediator $M$ on non-local particle $S$ uses entanglement $\varepsilon(R, \bar{R})$, or equivalently $\varepsilon(R, S)$. Once the mediator is created, it fully controls the transfer until its end at time $\tau_{E}$. Whenever mediator $M$ needs to access particle $R$, it uses $\varepsilon(M, R)$,

5 We use the term non-local in the sense of non-contact or distant. Note that some authors use this term in a different sense. 
and whenever it needs to access particle $\bar{R}$, it uses a chain of entanglements $\varepsilon(M, R)$ plus $\varepsilon(R, \bar{R})$. In total, entanglements $\varepsilon(R, \bar{R})$ and $\varepsilon(M, R)$ are sufficient for any transfer, that is no information transfer requires more than two entanglements in order to work.

\subsection{Information transfers with $R \neq S$}

In the information transfers with $R \neq S$ particle $R$ requires $\varepsilon(R, \bar{R})$ in order to create mediator $M$ on particle $S$. The mediator, then, does the remaining actions. If there were $\varepsilon(M, \bar{R})$, the mediator would have access to particle $\bar{R}$, but it would in no way have access to particle $R$. This would prevent the mediator from recognizing target particle $T=R$ and finishing its own existence when it is co-local with $T$. Entanglement $\varepsilon(M, \bar{R})$ is, therefore, ruled out. Consequently, the transfers with $R \neq S$ can work only with entanglements $\varepsilon(R, \bar{R})$ and $\varepsilon(M, R)$. Here we talk about 14 information transfers: $I_{9}$ to $I_{15}$, and $I_{17}$ to $I_{23}$.

\subsection{Information transfers $I_{26}, I_{27}, I_{30}$ and $I_{31}$}

Information transfers $I_{26}, I_{27}, I_{30}$ and $I_{31}$ satisfy $R=S=B$ and $\tau_{0} \leq \tau_{A}<\tau_{B} \leq \tau_{E}$. In these transfers mediator $M$ is co-local with particle $A$ only at time $\tau_{E}$, and is co-local with particle $B$ only at time $\tau_{0}$. Since $\tau_{A}<\tau_{E}$, mediator $M$ is at time $\tau_{A}$ not co-local with particle $A$. It means that $\triangle q$ is taken from particle $A$ non-locally. Similarly, since $\tau_{B}>\tau_{0}$, mediator $M$ is not co-local with particle $B$ at time $\tau_{B}$. Thus, $\Delta q$ is put to particle $B$ also non-locally. In total, the mediator requires non-local access to both particles $A$ and $B$. It is clear that one entanglement cannot link three participants, and therefore two entanglements are needed.

There are two pairs of entanglements which allow mediator $M$ to access both particles $A$ and $B: \varepsilon(M, A)$ plus $\varepsilon(A, B)$, and $\varepsilon(M, B)$ plus $\varepsilon(B, A)$. The problem with the first pair is that there is no entanglement $\varepsilon(B, A)$, and thus particle $B$ does not know the identity of particle $A$. It means that particle $B$ at time $\tau_{0}$ cannot create mediator $M$ with entanglement $\varepsilon(M, A)$. The pair $\varepsilon(M, A)$ plus $\varepsilon(A, B)$ is, therefore, ruled out. The other pair of entanglements, $\varepsilon(M, B)$ plus $\varepsilon(B, A)$, does not face the problem. When particle $B$ creates mediator $M$, it gives to the mediator the self-identifier, that is mediator $M$ is created with $\varepsilon(M, B)$. We see that $\varepsilon(M, R)$ plus $\varepsilon(R, \bar{R})$ are the minimum set of entanglements which makes transfers $I_{26}, I_{27}, I_{30}$, and $I_{31}$ work.

\subsection{Information transfers $I_{1}$ and $I_{3}$}

Information transfers $I_{1}$ and $I_{3}$ satisfy $R=S=A$ and $\tau_{0}=\tau_{A} \leq \tau_{B}<\tau_{E}$. At time $\tau_{0}$ they do everything related to particle $A$ : initiator $A$ creates mediator $M$ on itself, and the mediator takes $\triangle q$ from co-local particle $A$. The remaining actions are related only to particle $B$. Mediator $M$ is co-local with particle $B$ only at time $\tau_{E}$. Since $\tau_{B}<\tau_{E}$, the mediator is not co-local with particle $B$ at time $\tau_{B}$ when it puts $\Delta q$ to the particle. In other words, the mediator requires non-local access to particle $B$. It can only be provided by the initiator of the transfer, that is $\varepsilon(A, B)$ is required. In that case, particle $A$, besides knowing its own identity, also knows the identity of particle $B$. Therefore, particle $A$ can create the mediator either with $\varepsilon(M, A)$ or with $\varepsilon(M, B)$. In the former case, whenever mediator $M$ wants to access particle $B$ to do any of the remaining actions, it uses a chain of entanglements $\varepsilon(M, A)$ plus $\varepsilon(A, B)$, whereas in the latter case it uses only $\varepsilon(M, B)$. Both variants, therefore, allow information transfers $I_{1}$ and $I_{3}$ to work. 
6.6 Information transfers $I_{5}$ and $I_{7}$

Information transfers $I_{5}$ and $I_{7}$ satisfy $R=S=A$ and $\tau_{0}<\tau_{A} \leq \tau_{B}<\tau_{E}$. In these transfers mediator $M$ is co-local with particle $A$ only at time $\tau_{0}$ and is co-local with particle $B$ only at time $\tau_{E}$. Since $\tau_{0}<\tau_{A}$, mediator $M$ is not co-local with particle $A$ at time $\tau_{A}$, and thus $\triangle q$ must be taken from particle $A$ non-locally. Similarly, since $\tau_{B}<\tau_{E}$, the mediator is not co-local with particle $B$ at time $\tau_{B}$, and thus $\Delta q$ must be put to particle $B$ non-locally. In total, mediator $M$ requires non-local access to both particles $A$ and $B$. One entanglement cannot link three participants, and thus two entanglements are needed.

There are two pairs of entanglements which allow mediator $M$ to access both particles $A$ and $B: \varepsilon(M, B)$ plus $\varepsilon(B, A)$, and $\varepsilon(M, A)$ plus $\varepsilon(A, B)$. In the first case, there is no entanglement $\varepsilon(A, B)$, and thus particle $A$ does not know the identity of particle $B$. Consequently, particle $A$ cannot create mediator $M$ with entanglement $\varepsilon(M, B)$. The pair of entanglements $\varepsilon(M, B)$ plus $\varepsilon(B, A)$ is, therefore, ruled out. The other pair of entanglements, $\varepsilon(M, A)$ plus $\varepsilon(A, B)$, does not face this problem. When particle $A$ creates mediator $M$, it gives to the mediator the self-identifier, that is $M$ is created with $\varepsilon(M, A)$. We see that $\varepsilon(M, R)$ plus $\varepsilon(R, \bar{R})$ are the minimum set of entanglements which makes transfers $I_{5}$ and $I_{7}$ work.

\subsection{Information transfers $I_{2}, I_{4}$ and $I_{6}$}

Information transfers $I_{2}, I_{4}$ and $I_{6}$ satisfy $R=S=A$ and $\tau_{0} \leq \tau_{A} \leq \tau_{B}=\tau_{E}$. In these transfers mediator $M$ is co-local with particle $A$ only at time $\tau_{0}$ and is co-local with particle $B$ only at time $\tau_{E}$. Since $\tau_{B}=\tau_{E}$, the mediator is at time $\tau_{B}$ co-local with particle $B$. Therefore, it puts $\triangle q$ to particle $B$ locally and no entanglement is required for this action. Moreover, the identity of particle $T=B$ may be unknown until the mediator hits the particle at time $\tau_{E}$. If it is really unknown, then the mediator does not search in the last phase of its lifetime for the specific particle $B$. Thus, mediator $M$ does not require any entanglement for actions related to particle $B$. In information transfers $I_{4}$ and $I_{6}$ with $\tau_{0}<\tau_{A}$ mediator $M$ is not co-local with particle $A$ at time $\tau_{A}$, and thus $\Delta q$ must be taken from particle $A$ non-locally. This is not true about transfer $I_{2}$ with $\tau_{0}=\tau_{A}$ where mediator $M$ takes $\triangle q$ from particle $A$ locally. In total, information transfer $I_{2}$ can work without any entanglement, and transfers $I_{4}$ and $I_{6}$ can work with single entanglement $\varepsilon(M, A)$ which is created by initiator $A$ at time $\tau_{0}$ together with mediator $M$.

\subsection{Information transfers $I_{25}, I_{28}$ and $I_{29}$}

Information transfer $I_{25}$ satisfies $R=S=B, T=A$, and $\tau_{0}=\tau_{A}=\tau_{B}<\tau_{E}$. The transfer can be obtained from transfer $I_{1}$ (satisfies $R=S=A, T=B$, and $\tau_{0}=\tau_{A}=\tau_{B}<\tau_{E}$ ) by the exchange of $A \leftrightarrow B$ in the role distribution and in the timing. Therefore, when we exchange $A$ and $B$ (i.e., (from) particle $A \leftrightarrow$ (to) particle $B, \tau_{A} \leftrightarrow \tau_{B}$ ) in the argumentation why transfer $I_{1}$ requires either $\varepsilon(R, \bar{R})$ plus $\varepsilon(M, R)$ or $\varepsilon(R, \bar{R})$ plus $\varepsilon(M, \bar{R})$, we get an argumentation why transfer $I_{25}$ also requires either $\varepsilon(R, \bar{R})$ plus $\varepsilon(M, R)$ or $\varepsilon(R, \bar{R})$ plus $\varepsilon(M, \bar{R})$.

The very same procedure can also be applied to transfers $I_{28}$ and $I_{29}$. This is because $I_{28}$ and $I_{29}$ can be obtained by the exchange of $A \leftrightarrow B$ from transfers $I_{4}$ and $I_{5}$, respectively. In total, we get that in order to work transfer $I_{28}$ requires only $\varepsilon(M, R)$, and that transfer $I_{29}$ requires two entanglements, namely $\varepsilon(R, \bar{R})$ and $\varepsilon(M, R)$. 
6.9 Summary of entanglements

Here we summarize several important facts about entanglements. Firstly, the requirements of information transfers for entanglements, as derived in the preceding text, are as follows:

(a) Information transfer $I_{2}$ does not require any entanglement in order to work.

(b) Information transfers $I_{4}, I_{6}$ and $I_{28}$ require one entanglement in order to work, namely $\varepsilon(M, R)$.

(c) Information transfers $I_{1}, I_{3}$ and $I_{25}$ can work in two ways. They require either $\varepsilon(R, \bar{R})$ and $\varepsilon(M, R)$, or $\varepsilon(R, \bar{R})$ and $\varepsilon(M, \bar{R})$,

(d) The remaining 21 information transfers, i.e., $I_{i}$ with $i \in \mathbb{F} \backslash\{1,2,3,4,6,25,28\}$, require two entanglements in order to work, namely $\varepsilon(R, \bar{R})$ and $\varepsilon(M, R)$.

Each information transfer, in order to satisfy the minimum number of entanglements, works in the way that it always uses only the required entanglements. In other words, even if other entanglements existed, such as $\varepsilon(\bar{R}, R)$, they would be ignored by the transfer. The minimum number of entanglements also dictates that there are no duplicities of entanglements. It means that there is maximally one entanglement from a given particle to another given particle. It suggests that a single entanglement between two particles can serve as $\varepsilon(R, \bar{R})$ for various information transfers. It means that several transfers are interrelated. Finally, in every transfer, except $I_{2}$, one entanglement is created. Since it is either $\varepsilon(M, R)$ or $\varepsilon(M, \bar{R})$, the created entanglement is only temporary. It is destroyed no later than at the end of the transfer when mediator $M$ is destroyed. None of the transfers in order to work require for any of its participant to have more than one outgoing entanglement. It seems that $N_{\varepsilon}^{\max }=1$. However, we hesitate to conclude this fact because so far we have not ruled out composite mediators with internal entanglements.

\subsection{Local information transfer}

Information transfers can be fully local only when they do not require any entanglement in order to work. According to the entanglements that each information transfer requires, there is only one information transfer with such a property, namely transfer $I_{2}$. In other words, transfer $I_{2}$ is the only information transfer which can be fully local. No requirement for entanglements makes $I_{2}$ be the least restrictive transfer from all the information transfers, at least as far as entanglements are concerned. Therefore, we expect transfer $I_{2}$ to represent a very common process, perhaps a very common fundamental interaction.

\subsection{Interaction of every particle with each other}

Some information transfers can represent interactions where every particle interacts with each other. Let particles interact only by an information transfer which in order to work requires entanglement $\varepsilon(A, B)$ or $\varepsilon(B, A)$. Such a transfer works only between $A$ and $B$, the particles determined by the required entanglement. Definition 1 guarantees that the transfer ignores all the other particles in the Universe. It means that in a group of $n$ particles, every particle can interact with each other only when every two particles of the group are entangled, i.e., when there are at least $n(n-1) / 2$ entanglements. However, there are maximally $n \cdot N_{\varepsilon}^{\max }$ outgoing entanglements in the group of $n$ particles. In a group of $n \leq 2 N_{\varepsilon}^{\max }+1$ particles, it is $n \cdot N_{\varepsilon}^{\max } \geq n(n-1) / 2$, and thus there can exist an entanglement configuration such that every particle of the group can interact with each other. On the other hand, for a group with 
$n>2 N_{\varepsilon}^{\max }+1$ particles, it is

$$
n \cdot N_{\varepsilon}^{\max }<\frac{n \cdot(n-1)}{2}
$$

and consequently there are too few entanglements to make possible an interaction of every particle with each other. The statement is true for every transfer which in order to work requires $\varepsilon(A, B)$ or $\varepsilon(B, A)$. Information transfers $I_{2}, I_{4}, I_{6}$, and $I_{28}$ are the only transfers which do not need entanglements $\varepsilon(A, B)$ and $\varepsilon(B, A)$. Thus, they are the only transfers which can provide interaction of every particle with each other in a particle group of any size.

\subsection{Example of information transfer}

In this section, we choose one information transfer and show in detail how it works and what entanglements it requires. We show it on transfer $I_{14}$ which satisfies $R=T=B, S=A$ and $\tau_{0}<\tau_{A}<\tau_{B}=\tau_{E}$.

The initiator of information transfer $I_{14}$, particle $B$, at time $\tau_{0}$ needs to create immediately mediator $M_{14}$ on particle $A$. Since particle $A$ and particle $B$ are generally not co-local, particle $B$ can do it only via an entanglement, namely that it needs $\varepsilon(B, A)$. It means the existence of the entanglement $\varepsilon(B, A)$ is a necessary condition for the information transfer $I_{14}$ to work, and without the entanglement the transfer could not even start. Thus, at time $\tau_{0}$ particle $B$ uses its only entanglement $\varepsilon(B, A)$, creates mediator $M_{14}$ on particle $A$, and immediately hands control over the information transfer to the mediator.

When particle $B$ at time $\tau_{0}$ uses entanglement $\varepsilon(B, A)$ to create mediator $M_{14}$, all the participants of the information transfer in the sense of definition 1 are determined. Firstly, the identity of particle $A$ is known, the particle from which the information $\Delta q$ will be taken at time $\tau_{A}$. Secondly, the identity of particle $B$ is also known, that is the particle to which the information $\Delta q$ will be transferred and where the mediator will finish its own existence. Mediator $M_{14}$, therefore, needs to know these identities in order to interact with the right particles during its lifetime. One way to know the identities is to create two new entanglements, $\varepsilon\left(M_{14}, A\right)$ and $\varepsilon\left(M_{14}, B\right)$. There is, however, another way to know both identities, namely to create only one new entanglement $\varepsilon\left(M_{14}, B\right)$. The latter way creates fewer entanglements, and thus is preferred by postulate 1 . In that case, mediator $M_{14}$ has access to particle $B$ via entanglement $\varepsilon\left(M_{14}, B\right)$, and has access to particle $A$ via a chain of entanglements $\varepsilon\left(M_{14}, B\right)$ plus $\varepsilon(B, A)$.

Then the mediator starts to propagate through space. Later at time $\tau_{A}$, mediator $M_{14}$ is generally not co-local with particle $A$, and thus uses entanglements $\varepsilon\left(M_{14}, B\right)$ and $\varepsilon(B, A)$ in order to transfer $\triangle q$ from particle $A$ to itself. Time $\tau_{A}$ is the last moment when particle $A$ participates in the transfer.

Then, mediator $M_{14}$ starts to look for the target particle $T=B$. The mediator performs it in the way that whenever it becomes co-local with a particle, it reads its self-identifier and compares it with $\varepsilon\left(M_{14}, B\right)$, that is it compares it with the identifier of particle $B$ stored in itself. If the identifiers are not the same, then in order to do another check the mediator waits until another particle becomes co-local with the mediator. On the other hand, if the identifiers are the same, then mediator $M_{14}$ has finally found particle $B$. Now, it is time $\tau_{B}=\tau_{E}$. The mediator immediately transfers $\triangle q$ from itself to co-local particle $B$ and finishes its own existence. 


\section{Basics of dynamics}

The most important characteristic of each interaction is how it influences particles involved in the interaction. We dedicate this section to answering the spatial part of this question for each information transfer within three-dimensional space, that is in postulate 2 we apply $d=3$. The principal goal of this paper is to try to map the fundamental interactions onto the fundamental information transfers as fast as possible. Therefore, we do not try to build dynamics which would work in all circumstances, and we focus only on some special cases. Firstly, in order to emphasize the underlying asymmetry between particle $A$ and particle $B$, we examine only the transfers of information $\triangle q$ from particle $A$ to particle $B$ and not vice versa. Secondly, we assume that some particles are point-like. Namely, particle $S$ is point-like at time $\tau_{0}$, particles $A$ and $B$ are point-like at time $\tau_{A}$, and particle $T$ is point-like at time $\tau_{E}$. Thirdly, we assume that the speed of mediators (and their parts) is during time interval $\left(\tau_{0}, \tau_{A}\right)$ constant. Fourthly, for each transfer with $\tau_{0}<\tau_{A}$ that does not finish at time $\tau_{A}$ by hitting particle $T$, that is for each transfer that satisfies $\tau_{0}<\tau_{A}<\tau_{E}$, we assume $\tau_{A}-\tau_{0}$ is constant. Fifthly, for each transfer with $\tau_{A}<\tau_{B}$ that does not finish at time $\tau_{B}$ by hitting particle $T$, that is for each transfer that satisfies $\tau_{A}<\tau_{B}<\tau_{E}$, we assume $\tau_{B}-\tau_{A}$ is constant. Finally, if no speed of participants appears automatically, we usually examine only a static situation.

\subsection{Movement and momentum of mediators}

The movement of mediator $M$ depends on its momentum $\mathbf{p}_{M}$. However, if the movement of the mediator had always the same direction as the momentum of the mediator, then all contact forces would be only repulsive. Therefore, ${ }^{6}$ we suppose that the movement of a mediator (or the movement of its part) depends on the momentum of the mediator (or on the momentum of the corresponding part), except for the sign of its direction. That is, the velocity of the mediator (or of its part) is either parallel or antiparallel to the corresponding momentum and the specific choice of the sign is a characteristic quantity for each process.

\subsection{Internal structure of mediators}

We have assumed particles $A$ and $B$ are structureless, however, we have not done that for the mediators. Here we show why. In the transfers with $\tau_{0}<\tau_{A}$ mediator $M$ during time interval $\left(\tau_{0}, \tau_{A}\right)$ satisfies equation (14), that is $\mathbf{p}_{M}\left(\tau_{0 A}\right)=\mathbf{0}$. Similarly, in the transfers with $\tau_{B}<\tau_{E}$ the mediator during $\left(\tau_{B}, \tau_{E}\right)$ satisfies equation (16), that is $\mathbf{p}_{M}\left(\tau_{B E}\right)=\mathbf{0}$. In each of these cases, if the mediator has no internal structure, then it is not moving, and consequently it is not transferring information anywhere. The only way to make it move through space toward distant particle $T$ is to assume that the mediator has an internal structure, that is it is composed of several parts (hereafter called mediatorinos). The minimalistic postulate 1 dictates to express the mediator as a sum of two mediatorinos, symbolically written as $M=m_{1}+m_{2}$. In that case, at every time $t \in\left(\tau_{0}, \tau_{A}\right) \cup\left(\tau_{B}, \tau_{E}\right)$ there must be

$$
q_{M}(t)=q_{m_{1}}(t) \oplus q_{m_{2}}(t)=q_{0} .
$$

Since $q$ can be any transferable conserved quantity, $m_{1}$ and $m_{2}$ must have the mutually opposite quantum numbers, such as electric charge, baryon number, lepton number, and so on. If mediatorino $m_{1}$ represents a particle, then mediatorino $m_{2}$ must be its antiparticle, i.e.,

${ }^{6}$ Here we are motivated by the (so far not proved) fact that attractive gravity is a contact force. 
$M=m_{1}+\bar{m}_{1}$. Quantity $q$ can also be momentum $\mathbf{p}$. Then, regardless of whether in the process the velocities of $m_{1}$ and $m_{2}$ are both parallel or both antiparallel to the momentum of $m_{1}$ and $m_{2}$, respectively, mediatorinos $m_{1}$ and $m_{2}$ must go in the mutually opposite directions. Although using $m_{1}$ and $m_{2}$ works mathematically, we should be careful in its interpretation: in mediator $M$ mediatorinos $m_{1}$ and $m_{2}$ are created simultaneously, behave in the complementary way, and maybe in some cases are even annihilated simultaneously. Therefore, we never should think of one mediatorino without taking into consideration the other mediatorino. In the transfers with $\tau_{A}<\tau_{B}$ mediator $M$ during time interval $\left(\tau_{A}, \tau_{B}\right)$ satisfies equation (12), i.e., $\mathbf{p}_{M}\left(\tau_{A B}\right)=\triangle \mathbf{p}$. This allows the mediator to move through space and transfer information even if it has no internal structure. That is, even if the mediator is composed of a single mediatorino, symbolically written as $M=m$.

Postulate 1 dictates to minimize the number of parts of a mediator passively. It means that the number of mediatorinos increases only when it is necessary and never proactively decreases (except for the very end of the transfer). Therefore, in the transfers with $\tau_{0}<\tau_{A}$ mediator $M$ is composed of two mediatorinos in every of its time phases. In the transfers with $\tau_{0}=\tau_{A}<\tau_{B}$ mediator $M$ is during $\left(\tau_{A}, \tau_{B}\right)$ composed of one mediatorino, nevertheless, if the transfers also satisfy $\tau_{B}<\tau_{E}$, the mediator is during $\left(\tau_{B}, \tau_{E}\right)$ composed of two mediatorinos. Finally, in the transfers with $\tau_{0}=\tau_{A}=\tau_{B}<\tau_{E}$ the mediator is composed of two mediatorinos. In total, while for transfers $I_{i}$ with $i \in\{2,3,10,11,18,19,26,27\}$ the number of mediatorinos $n_{i, m}$ within $\tau_{A B}$ satisfies

$$
n_{i, m}\left(\tau_{A B}\right)=1,
$$

for the remaining times (i.e., for $t=\tau_{0 A}$ or $t=\tau_{B E}$ ) or for the remaining transfers (i.e., with $i \in \mathbb{F} \backslash\{2,3,10,11,18,19,26,27\})$ the number of mediatorinos satisfies

$$
n_{i, m}(t)=2 .
$$

Whenever mediator $M$ is composed of two mediatorinos, the mediator, besides $\varepsilon(M, R)$, may contain maximally two internal entanglements, namely $\varepsilon\left(m_{1}, m_{2}\right)$ and $\varepsilon\left(m_{2}, m_{1}\right)$. Moreover, no matter whether $\varepsilon(M, R)$ is implemented as $\varepsilon\left(m_{1}, R\right)$ and $\varepsilon\left(m_{2}, R\right)$ or only one of them (which is preferred by postulate 1 ), we can conclude that $N_{\varepsilon}^{\max } \leq 2$.

\subsection{Determination of vectorial $\triangle \mathbf{q}$}

Conserved quantity can be vectorial or pseudovectorial. In such a case, it is important to know how the direction of $\Delta \mathbf{q}$ is determined. Let us assume it is determined by the location of the participants of the transfer. Since the minimum memory complexity does not allow storing historical data, such as locations, $\triangle \mathbf{q}$ must be determined by the location the participants have just at the time of the determination of $\triangle \mathbf{q}$, that is at time $\tau_{A}$. Mediator $M$ takes $\triangle \mathbf{q}$ from particle $A$, and thus we may naturally assume that $\Delta \mathbf{q}$ depends on the location of particle $A$ and the location of mediatorino(s) within the mediator. This supports also the fact that incorporating the location of particle $B, \mathbf{x}_{B}\left(\tau_{A}\right)$, into the determination of $\triangle \mathbf{q}$ would require an extra entanglement at least for transfers $I_{2}$ and $I_{6}$. This would go against the minimum memory complexity. Therefore, we assume that the location of particle $B$ does not participate in the determination of $\triangle \mathbf{q}$, that is $\triangle \mathbf{q}$ is based on $\mathbf{x}_{A}\left(\tau_{A}\right)$ and $\mathbf{x}_{m}\left(\tau_{A}\right)$. We have the following dynamic law:

Law 1 (Determination of $\triangle \mathbf{q}$ ) In the volume of mediatorino $m$ and in the volume of particle A are at time $\tau_{A}$ chosen points $\mathbf{x}_{m}^{c}\left(\tau_{A}\right)$ and $\mathbf{x}_{A}^{c}\left(\tau_{A}\right)$, respectively. If mediatorino $m$ is co-local with particle $A$ or $B$, then $\mathbf{x}_{m}^{c}\left(\tau_{A}\right)$ is chosen within the volume of that co-local particle. The 
value of $\triangle \mathbf{q}$ is, then, determined in the way that it has a constant size $\Delta q$, and its direction is determined in the following way:

(a) If $\mathbf{x}_{m}^{c}\left(\tau_{A}\right)=\mathbf{x}_{A}^{c}\left(\tau_{A}\right)$, then the direction of $\triangle \mathbf{q}$ is chosen randomly and all the possible directions have the same probability of being chosen.

(b) If $\mathbf{x}_{m}^{c}\left(\tau_{A}\right) \neq \mathbf{x}_{A}^{c}\left(\tau_{A}\right)$, then the direction of $\triangle \mathbf{q}$ is chosen along the line

$$
\mathbf{l}=\mathbf{x}_{m}^{c}\left(\tau_{A}\right)-\mathbf{x}_{A}^{c}\left(\tau_{A}\right)
$$

created from points $\mathbf{x}_{m}^{c}\left(\tau_{A}\right)$ and $\mathbf{x}_{A}^{c}\left(\tau_{A}\right)$.

The dynamic law can be implemented in the following way: At time $\tau_{A}$ mediatorino $m$ chooses in its volume a point, $\mathbf{x}_{m}^{c}\left(\tau_{A}\right)$, and provides its location to particle $A$ while asking it for $\triangle \mathbf{q}$. Then particle $A$ chooses in its volume a point, $\mathbf{x}_{A}^{c}\left(\tau_{A}\right)$, which for point-like particle $A$ satisfies $\mathbf{x}_{A}^{c}\left(\tau_{A}\right)=\mathbf{x}_{A}\left(\tau_{A}\right)$. Particle $A$, then, evaluates the law, takes $\triangle \mathbf{q}$ from that part of itself which is located at point $\mathbf{x}_{A}^{c}\left(\tau_{A}\right)$, and still at time $\tau_{A}$ gives it to the mediatorino while returning control over the transfer. This is what we mean by saying that mediator $M$ takes $\triangle \mathbf{q}$ from particle $A$.

The direction of $\triangle \mathbf{q}$ is for $\mathbf{x}_{m}^{c}\left(\tau_{A}\right) \neq \mathbf{x}_{A}^{c}\left(\tau_{A}\right)$ defined uniquely, except for its sign. Since $\mathbf{v}_{m}\left(\tau_{A B}\right)$ is either parallel or antiparallel to momentum $\triangle \mathbf{p}$ (which is a special case of $\triangle \mathbf{q}$ ), the direction of $\mathbf{v}_{m}\left(\tau_{A B}\right)$ is also defined uniquely, except for its sign. The role of velocity $\mathbf{v}_{m}\left(\tau_{A B}\right)$ is to propagate mediatorino $m$ during time interval $\left(\tau_{A}, \tau_{B}\right)$ through space. Since the mediatorino is at time $\tau_{A}$ at point $\mathbf{x}_{m}^{c}\left(\tau_{A}\right)$ and since $\mathbf{v}_{m}\left(\tau_{A B}\right)$ is either parallel or antiparallel to line $\mathbf{l}$ connecting $\mathbf{x}_{m}^{c}\left(\tau_{A}\right)$ and $\mathbf{x}_{A}^{c}\left(\tau_{A}\right)$, the mediatorino moves during time interval $\left[\tau_{A}, \tau_{B}\right]$ only on line $\mathbf{l}$. Its location can be expressed as

$$
\mathbf{x}_{m}(t)=\mathbf{x}_{m}^{c}\left(\tau_{A}\right)+\mathbf{v}_{m}\left(\tau_{A B}\right) \cdot\left(t-\tau_{A}\right)
$$

where $t \in\left[\tau_{A}, \tau_{B}\right]$.

\subsection{Probabilistic description}

Mediatorino $m$ can be at time $\tau_{A}$ in many places. It means that the direction of $\Delta \mathbf{q}$ can be various. Moreover, there can be a lot of determinations of $\Delta \mathbf{q}$ at the same time. It suggests developing a probabilistic quantity. Moreover, using probabilistic quantities as the base for dynamics should guarantee that the model will never be divergent. Let us quantify how probable it is within a single performance of transfer $I_{i}$ for a particle at point $\mathbf{x}$ that the change of quantity $\mathbf{q}$ is a specific vector $\mathbf{q}$. We introduce $Q_{i}(\mathbf{x}, \mathbf{q}) \geq 0$, a distribution of changes of quantity $\mathbf{q}$, such that $d P_{i}$ in equation

$$
d P_{i}(\mathbf{x}, \mathbf{q})=Q_{i}(\mathbf{x}, \mathbf{q}) d q d \Omega
$$

represents that probability. The infinitesimal solid angle $d \Omega$ is created by infinitesimal variations of the end point of vector $\mathbf{q}$, keeping the size of the vector constant and keeping the origin point of $\mathbf{q}$ fixed at point $\mathbf{x}$. A field composed of $Q_{i}(\mathbf{x}, \mathbf{q})$ should be called $q$-distribution field (or simply distribution field) because for every point $\mathbf{x}$ in space it expresses a distribution of changes of quantity $\mathbf{q}$ for every possible vector $\mathbf{q}$.

\subsection{Directional distribution of quantity}

Quantity $Q_{i}(\mathbf{x}, \mathbf{q})$ is suitable for general fields. However, in this article we deal with transfers where due to law 1 the size of $\Delta \mathbf{q}$ is constant. In that case, for a fixed unit directional vector 
ê we can write

$$
Q_{i}(\mathbf{x}, q \cdot \hat{\mathbf{e}})=\delta(q-\Delta q) \cdot K_{i}(\mathbf{x}, \hat{\mathbf{e}}),
$$

where $K_{i}$ is a function of only position $\mathbf{x}$ and direction $\hat{\mathbf{e}}$. Then, it is convenient to integrate over the sizes of quantity $\mathbf{q}$ in order to reduce the number of parameters from six to five. Since

$$
\int_{0}^{\infty} Q_{i}(\mathbf{x}, q \cdot \hat{\mathbf{e}}) d q=K_{i}(\mathbf{x}, \hat{\mathbf{e}}),
$$

we can express probability $d P_{i}(\mathbf{x}, \hat{\mathbf{e}})$ that the change of quantity $\mathbf{q}$ has a specific direction $\hat{\mathbf{e}}$ as

$$
d P_{i}(\mathbf{x}, \hat{\mathbf{e}})=\left(\int_{0}^{\infty} Q_{i}(\mathbf{x}, q \cdot \hat{\mathbf{e}}) d q\right) d \Omega=K_{i}(\mathbf{x}, \hat{\mathbf{e}}) d \Omega .
$$

Quantity $K_{i}(\mathbf{x}, \hat{\mathbf{e}})$ is, therefore, called directional distribution of a change of quantity $\mathbf{q}$.

Since not every $\triangle \mathbf{q}$ taken from a given particle $A$ arrives to a given particle $B$ in every transfer, we need two distributions: $K_{i, A}\left(\mathbf{x}_{A}, \hat{\mathbf{e}}\right)$, the directional distribution of a change of $\mathbf{q}$ on particle $A$, and $K_{i, B}\left(\mathbf{x}_{B}, \hat{\mathbf{e}}\right)$, the directional distribution of a change of $\mathbf{q}$ on particle $B$ which originated from particle $A$. Within a complete single performance of information transfer $I_{i}$ with

$$
\triangle \mathbf{q}=\triangle q \cdot \triangle \hat{\mathbf{q}}
$$

information $\mathbf{q}$ on particle $A$ is at time $\tau_{A}$ changed by $-\triangle \mathbf{q}$ because $\triangle \mathbf{q}$ is taken from particle $A$. That is, in $K_{i, A}\left(\mathbf{x}_{A}, \hat{\mathbf{e}}\right)$ it is $\hat{\mathbf{e}}=-\triangle \hat{\mathbf{q}}$. On the other hand, $\mathbf{q}$ on particle $B$ is at time $\tau_{B}$ changed by $\triangle \mathbf{q}$ because $\triangle \mathbf{q}$ is put to particle $B$. That is, in $K_{i, B}\left(\mathbf{x}_{B}, \hat{\mathbf{e}}\right)$ there is $\hat{\mathbf{e}}=\triangle \hat{\mathbf{q}}$. Thus, a transfer of $\triangle \mathbf{q}$ from particle $A$ to particle $B$ interrelates $K_{i, A}\left(\mathbf{x}_{A},-\triangle \hat{\mathbf{q}}\right)$ with $K_{i, B}\left(\mathbf{x}_{B}, \triangle \hat{\mathbf{q}}\right)$. This is true for every possible direction $\triangle \hat{\mathbf{q}}$.

Let us refine parameters of $K_{i, A}$ and $K_{i, B}$. Firstly, it is convenient to replace locations $\mathbf{x}_{A}$ and $\mathbf{x}_{B}$ by some characteristic distance $r$. Interestingly, for $K_{i, A}$ and $K_{i, B}$ are sometimes suitable different characteristic distances, hereafter represented by vector $\mathbf{r}_{S}$ and $\mathbf{r}_{T}$. Whenever required, these vectors will be explicitly defined. Thus, now we have $K_{i, A}\left(\mathbf{r}_{S}, \hat{\mathbf{e}}\right)$ and $K_{i, B}\left(\mathbf{r}_{T}, \hat{\mathbf{e}}\right)$. Directional vector $\hat{\mathbf{e}}$ can be in 3-dimensional euclidean space expressed by two spherical angles $\vartheta \in[0, \pi]$ and $\varphi \in[0,2 \pi]$ in the way that

$$
\hat{\mathbf{e}}=\hat{\mathbf{e}}(\vartheta, \varphi)=(\sin \vartheta \cos \varphi, \sin \vartheta \sin \varphi, \cos \vartheta) .
$$

Thus, we sometimes write $K_{i, A}\left(\mathbf{r}_{S}, \vartheta, \varphi\right)$ and $K_{i, B}\left(\mathbf{r}_{T}, \vartheta, \varphi\right)$.

For an initiated transfer $I_{i}$ we can express $P_{i, A}$, the probability that $\triangle \mathbf{q}$ is taken from particle $A$, and $P_{i, B}$, the probability that $\triangle \mathbf{q}$ is delivered to particle $B$. Both these probabilities are given by the integration of $K_{i}$ over the full solid angle $\Omega$ (i.e., over all the possible directions $\hat{\mathbf{e}}$ ),

$$
\begin{aligned}
P_{i, A} & =\int d P_{i, A}=\oint_{\Omega} K_{i, A} d \Omega \in[0,1], \\
P_{i, B} & =\int d P_{i, B}=\oint_{\Omega} K_{i, B} d \Omega \in[0,1] .
\end{aligned}
$$


For the integration over the full solid angle, we usually use spherical coordinates where $d \Omega=\sin \vartheta d \vartheta d \varphi$. Then, we symbolically write

$$
\begin{aligned}
P_{i, A} & =\oint_{\vartheta, \varphi} K_{i, A}\left(\mathbf{r}_{S}, \vartheta, \varphi\right) \sin \vartheta d \vartheta d \varphi, \\
P_{i, B} & =\oint_{\vartheta, \varphi} K_{i, B}\left(\mathbf{r}_{T}, \vartheta, \varphi\right) \sin \vartheta d \vartheta d \varphi .
\end{aligned}
$$

In the transfers with $\tau_{0}=\tau_{A}$ the value of $\Delta \mathbf{q}$ is taken from particle $A$ immediately at the beginning of the transfer, and thus the probability $P_{i, A}$ satisfies $P_{i, A}=1$. Similarly, in the transfers with $\tau_{0}<\tau_{A}<\tau_{E}$ the value of $\triangle \mathbf{q}$ is taken from particle $A$ always after a constant time $\tau_{A}-\tau_{0}$, i.e., $P_{i, A}=1$. In total, for transfers $I_{i}$ with $i \in \mathbb{F} \backslash\{4,12,20,28\}$,

$$
P_{i, A}=1 \text {. }
$$

In other words, the transfers with $\tau_{0}<\tau_{A}=\tau_{E}$ are the only transfers where $P_{i, A}$ may satisfy $P_{i, A}<1$. This is caused by the fact that mediatorino $m$ must first hit particle $T$ in order to take $\triangle \mathbf{q}$ from particle $A$, and hitting particle $T$ is obviously not guaranteed. The probability $P_{i, B}$ satisfies $P_{i, B}=1$ only for those transfers which satisfy equation (34) and where every $\triangle \mathbf{q}$ taken from particle $A$ is eventually put to particle $B$.

\subsection{Symmetry of directional distribution}

Directional distribution $K_{i, A}$ is fully determined by the geometry of transfer $I_{i}$ at time $\tau_{A}$. In transfers $I_{1}, I_{2}, I_{3}, I_{9}, I_{10}$, and $I_{11}$ with $\tau_{0}=\tau_{A}$ and $S=A$, and in transfers $I_{20}$ and $I_{28}$ with $\tau_{0}<\tau_{A}=\tau_{E}, T=A$, mediatorino $m$ is at time $\tau_{A}$ on particle $A$. Therefore, the direction of $\triangle \mathbf{q}$ is always determined by law 1-(a). It means that $K_{i, A}$ is spherically symmetric. For these transfers we will define $z$-axis as

$$
\mathbf{z}=\mathbf{x}_{B}\left(\tau_{B}\right)-\mathbf{x}_{A}\left(\tau_{A}\right) .
$$

In that case, the $z$-axis is the axis of symmetry of $K_{i, B}$. This definition of $z$-axis will be used also for $I_{4}$ and $I_{12}$ with $\tau_{0}<\tau_{A}=\tau_{E}$ and $S=A$, where both $K_{i, A}$ and $K_{i, B}$ become axis-symmetric with respect to the $z$-axis.

For the rest of the information transfers, $\Delta \mathbf{q}$ is always determined by law 1-(b). Since the distribution of possible $\mathbf{x}_{m}^{c}\left(\tau_{A}\right)$ is at time $\tau_{A}$ spherically symmetric with respect to $\mathbf{x}_{S}\left(\tau_{0}\right)$, and since particle $A$ is at time $\tau_{A}$ a point-like particle located at $\mathbf{x}_{A}\left(\tau_{A}\right)$, defining $z$-axis as

$$
\mathbf{z}=\mathbf{x}_{S}\left(\tau_{0}\right)-\mathbf{x}_{A}\left(\tau_{A}\right),
$$

makes $K_{i, A}$ be axis-symmetric with respect to that $z$-axis. According to both equations (35) and (36), particle $A$ is at time $\tau_{A}$ always on $z$-axis, and thus whenever convenient, we may, without loss of generality, choose $\mathbf{x}_{A}\left(\tau_{A}\right)=[0,0,0]$.

\subsection{Average behavior of information transfers}

Directional distribution $K_{i}$, though being exact, may not be suitable to get a fast impression of what is going on in information transfer $I_{i}$. Therefore, we use $K_{i}$ to express some familiar approximative quantities, provided operator $\oplus$ of the summation of (pseudo)vectorial quantity $\mathbf{q}$ is the standard vector addition. For each information transfer $I_{i}$ and every $n \in \mathbb{N} \cup\{0\}$ 
let us define $n$-rank tensor

$$
\Gamma_{i}^{j_{1} j_{2} \ldots j_{n}}(\mathbf{r})=\oint_{\Omega} K_{i}(\mathbf{r}, \hat{\mathbf{e}}) e^{j_{1}} e^{j_{2}} \cdot \ldots \cdot e^{j_{n}} d \Omega,
$$

where $j_{s} \in\{1,2,3\}$ for every $s \in\{1, \ldots, n\}$, and where $e^{j_{s}}$ is the $j_{s}$-th component of (dimensionless) unit vector $\hat{\mathbf{e}}$ in equation (31). For $n=0$, we get a scalar quantity which, as we can see in equations (32a) and (32b), expresses either the probability that $\triangle \mathbf{q}$ is taken from particle $A$ or the probability that $\triangle \mathbf{q}$ arrives onto particle $B$. For $n=1$, we get a vector quantity

$$
\Gamma_{i}^{j}(\mathbf{r})=\oint_{\Omega} K_{i}(\mathbf{r}, \hat{\mathbf{e}}) e^{j} d \Omega
$$

which is related to the mean force vector

$$
\left\langle\mathbf{F}_{i}(\mathbf{r})\right\rangle=\left(\left\langle F_{i}^{1}(\mathbf{r})\right\rangle,\left\langle F_{i}^{2}(\mathbf{r})\right\rangle,\left\langle F_{i}^{3}(\mathbf{r})\right\rangle\right)^{T} .
$$

If $\mathbf{q}$ is linear momentum (i.e., $\mathbf{q}=\mathbf{p}, \Delta \mathbf{q}=\Delta \mathbf{p}$ ), then the mean of the $j$-th component of the force is

$$
\left\langle F_{i}^{j}(\mathbf{r})\right\rangle=f_{i} \cdot \Gamma_{i}^{j}(\mathbf{r}),
$$

with $f_{i}>0$ being a shorthand (with the dimension of force) for

$$
f_{i}=n_{i, F} \cdot \Delta p \cdot\left\langle v_{i}\right\rangle,
$$

where $n_{i, F} \in\{1,2\}$ represents the number of mediatorinos that contribute to the force. If all the mediatorinos contribute to the force, then $n_{i, F}=n_{i, m}$, however, if only one mediatorino contributes, then $n_{i, F}=1$. The value $\Delta p$ in equation (41) is the $\Delta q$ quantity from law 1 . The value of $\left\langle v_{i}\right\rangle$ has three variants. While $\left\langle v_{i, A}\right\rangle$ is the mean frequency of taking $\triangle \mathbf{p}$ from particle $A,\left\langle v_{i, B}\right\rangle$ is the mean frequency of putting $\triangle \mathbf{p}$ onto particle $B$. Besides $\left\langle v_{i, A}\right\rangle$ and $\left\langle v_{i, B}\right\rangle$, we also define $\left\langle v_{i, 0}\right\rangle$ as the mean frequency of the initiation of transfer $I_{i}$. The value

$$
f_{i, 0}=n_{i, F} \cdot \Delta p \cdot\left\langle v_{i, 0}\right\rangle,
$$

then, represents an important (maximal) characteristic of transfer $I_{i} \cdot{ }^{7}$ Similarly, for $n=2$ we get a 2-rank tensor quantity

$$
\Gamma_{i}^{k l}(\mathbf{r})=\oint_{\Omega} K_{i}(\mathbf{r}, \hat{\mathbf{e}}) e^{k} e^{l} d \Omega,
$$

which can be used to define

$$
\left\langle D_{i}^{k l}(\mathbf{r})\right\rangle=\frac{f_{i}}{S_{k}} \cdot \Gamma_{i}^{k l}(\mathbf{r}) .
$$

If $f_{i}$ is again defined by equation (41) and if $S_{k}$ is a unit area perpendicular to $k$-th axis, then $\left\langle D_{i}^{k l}(\mathbf{r})\right\rangle$ is a quantity very similar to stress tensor. It differs from stress tensor in the following: while stress tensor may express push, pull, and their combinations, $\left\langle D_{i}^{k l}(\mathbf{r})\right\rangle$ expresses only one of them. In agreement with $K_{i} \geq 0$ we interpret $\left\langle D_{i}^{k l}(\mathbf{r})\right\rangle$ in the way that it expresses pull. A pull in various directions may cause dispersion, and thus $\left\langle D_{i}^{k l}(\mathbf{r})\right\rangle$ will be called mean dispersion tensor. Although equation (37) can be used to express any-rank tensor quantity, we do not examine $n \geq 3$.

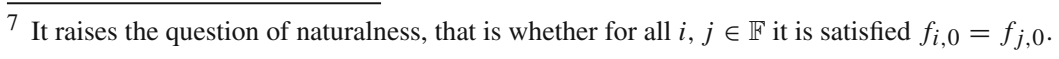


Directional distribution $K_{i, A}$ is either spherically symmetric, i.e., $K_{i, A}=K_{i, A}\left(\mathbf{r}_{S}\right)$, or axis symmetric with respect to $z$-axis, i.e., $K_{i, A}=K_{i, A}\left(\mathbf{r}_{S}, \vartheta\right)$. In both cases, $K_{i, A}$ is independent of $\varphi$. For all $i \in \mathbb{F}$ it immediately leads to

$$
\begin{aligned}
& \Gamma_{i, A}^{1}\left(\mathbf{r}_{S}\right)=0, \\
& \Gamma_{i, A}^{2}\left(\mathbf{r}_{S}\right)=0 .
\end{aligned}
$$

It means that the mean force $\left\langle\mathbf{F}_{i, A}\right\rangle$ is either a zero vector or it is parallel to $\pm \mathbf{z}$-axis. The independence of $K_{i, A}$ on angle $\varphi$ simplifies also the 2-rank tensor $\Gamma_{i, A}^{k l}$. Firstly, we decompose the integration over the full solid angle $\Omega$ into the integration over $\vartheta$ and the integration over $\varphi$. Since $K_{i, A}$ is independent of $\varphi$, it can be factored out of the integration over $\varphi$ and the integration can, then, be evaluated. The result is that $\Gamma_{i, A}^{k l}$ can be written as

$$
\Gamma_{i, A}^{k l}=\left(\begin{array}{ccc}
\Gamma_{i, A}^{11} & 0 & 0 \\
0 & \Gamma_{i, A}^{22} & 0 \\
0 & 0 & \Gamma_{i, A}^{33}
\end{array}\right),
$$

that is only diagonal elements of $\Gamma_{i, A}^{k l}$ can be non-zero. The diagonal elements and the trace of the tensor are

$$
\begin{aligned}
\Gamma_{i, A}^{11}=\Gamma_{i, A}^{22} & =\pi \int_{0}^{\pi} K_{i, A}\left(\mathbf{r}_{S}, \vartheta\right) \sin ^{3} \vartheta d \vartheta, \\
\Gamma_{i, A}^{33} & =2 \pi \int_{0}^{\pi} K_{i, A}\left(\mathbf{r}_{S}, \vartheta\right) \sin \vartheta \cos ^{2} \vartheta d \vartheta, \\
\operatorname{tr}\left(\Gamma_{i, A}^{k l}\right) & =\Gamma_{i, A}^{11}+\Gamma_{i, A}^{22}+\Gamma_{i, A}^{33}=\oint_{\Omega} K_{i, A} d \Omega .
\end{aligned}
$$

The last equation represents the contraction of 2-rank tensor $\Gamma_{i, A}^{k l}$ to 0 -rank tensor $\Gamma_{i, A}$. In the transfers with $\tau_{B}<\tau_{E}$ and in the transfers with $\tau_{0}<\tau_{A}=\tau_{E}$ all $\triangle \mathbf{q}$ taken from particle $A$ is eventually put to particle $B$, and thus these transfers work without any loss. It can be expressed in the way that $K_{i, B}(\mathbf{r}, \hat{\mathbf{e}})=K_{i, A}(\mathbf{r},-\hat{\mathbf{e}})$. For these transfers,

$$
\begin{aligned}
& \Gamma_{i, B}^{1}\left(\mathbf{r}_{T}\right)=0, \\
& \Gamma_{i, B}^{2}\left(\mathbf{r}_{T}\right)=0, \\
& \Gamma_{i, B}^{k l}\left(\mathbf{r}_{T}\right)=0 \text { for } k \neq l .
\end{aligned}
$$

Thus, in the following text we will calculate for most information transfers only $\Gamma_{i, A}^{3}, \Gamma_{i, B}^{3}$ and diagonal components of $\Gamma_{i, A}^{k l}\left(\mathbf{r}_{S}\right)$ and $\Gamma_{i, B}^{k l}\left(\mathbf{r}_{T}\right)$.

\subsection{Variants of $K_{i}$}

Directional distribution $K_{i}$ depends on the specific quantity q. It may also depend on velocity $\mathbf{v}_{m}\left(\tau_{A B}\right)$ because during time interval $\left(\tau_{A}, \tau_{B}\right)$ mediatorino $m$ holds information $\triangle \mathbf{q}$. Law 1 tells us the direction of $\triangle \mathbf{q}$, except for its sign. Similarly, the velocity of mediatorino $\mathbf{v}_{m}\left(\tau_{A B}\right)$ is either parallel or antiparallel to $\triangle \mathbf{q}$, and thus is defined, except for its sign, as well. These ambiguities produce several variants of $K_{i}$, and each of them will be labeled by a combination of signs in its superscript, e.g., $K_{i, B}^{+-}$. All the quantities that are derived from a specific $K_{i}$, such as $\Gamma_{i}$, will inherit its signs. 
The orientation of $\Delta \mathbf{q}$ at time $\tau_{A}$ is represented by quantity $s_{q}$ satisfying

$$
s_{q} \in\{-1,+1\} \text {. }
$$

Its sign will be the first sign in the superscript of $K_{i}$. Since we expect $s_{q}$ to be a characteristic of a process, we need some universal definition. We choose that the orientation of $\Delta \mathbf{q}$ will be related to particle $A$ which plays an important role at the moment of the determination of $\triangle \mathbf{q}$. The meaning of the sign is as follows: the sign + expresses that $\Delta \mathbf{q}$ points away from particle $A$, while the sign - expresses that $\triangle \mathbf{q}$ points toward particle $A$. In other words, in the case of law 1-(b), the signs + and - express that $\Delta \mathbf{q}$ is parallel and antiparallel to vector $\mathbf{I}$ from equation (24), respectively. In the case of law 1-(a), the situation with $\triangle \mathbf{q}$ pointing away from point-like particle $A$ is at time $\tau_{A}$ indistinguishable from $\triangle \mathbf{q}$ pointing toward point-like particle $A$. Therefore, in that case, if $\tau_{A}<\tau_{B}$, we define $s_{q}$ as the orientation of $\triangle \mathbf{q}$ at time $\tau_{A}+d t$ when mediatorino $m$ leaves particle $A$. If $\tau_{A}=\tau_{B}$, then there is no good definition of the orientation of $\Delta \mathbf{q}$, which is, in fact, a consequence of the symmetry with respect to the change of the sign of $\Delta \mathbf{q}$. Whatever value of $s_{q}$ we use in that case, it does not say anything about the direction of $\triangle \mathbf{q}$.

The orientation of $\mathbf{v}_{m}\left(\tau_{A B}\right)$, as determined at time $\tau_{A}$, is represented by quantity $s_{v}$ satisfying

$$
s_{v} \in\{-1,+1\} \text {. }
$$

Its sign will be the second sign in the superscript of $K_{i}$. Similar to the orientation of $\Delta \mathbf{q}$, signs + and - specify that $\mathbf{v}_{m}\left(\tau_{A B}\right)$ points away from or toward particle $A$, respectively. For $\tau_{A}=\tau_{B}$ there is no $\tau_{A B}$, and thus there is no good definition for $s_{v}=+1$, neither it is for $s_{v}=-1$. The transfers are, therefore, symmetric with respect to the change of the sign of $s_{v}$. In the case of law 1-(b), if $\tau_{A}<\tau_{B}$, then the signs + and - express that $\mathbf{v}_{m}\left(\tau_{A B}\right)$ is parallel and antiparallel to vector $\mathbf{I}$ from equation (24), respectively. In the case of law 1-(a), if $\tau_{A}<\tau_{B}$, then at $\tau_{A}+d t$ velocity $\mathbf{v}_{m}\left(\tau_{A B}\right)$ makes mediatorino $m$ move always away from particle $A$. It means that $s_{v}=-1$ does not exist in these transfers. The orientation of $\mathbf{v}_{m}\left(\tau_{A B}\right)$ is independent of the orientation of $\triangle \mathbf{q}$. Moreover, $\mathbf{v}_{m}\left(\tau_{A B}\right)$ can influence an information transfer only at $\tau_{A B}$, that is later than at time $\tau_{A}$. Consequently, the orientation of $\mathbf{v}_{m}\left(\tau_{A B}\right)$ does not influence $K_{i, A}$ which is a quantity describing the distribution of a change of $\mathbf{q}$ at time $\tau_{A}$. For that reason the orientation of $\mathbf{v}_{m}\left(\tau_{A B}\right)$ represented by the second superscript sign will occur only in $K_{i, B}$.

At time $\tau_{A}$ each of vectors $\triangle \mathbf{q}$ and $\mathbf{v}_{m}\left(\tau_{A B}\right)$ points toward or away from the current location of particle $A$. However, after some motion of particle $A$, this may no longer be true: the vectors may no point toward or away from the current location of particle $A$. Consequently, both the signs are valid at time $\tau_{A}$ and not necessarily at any later time.

Each $K_{i, A}$ has maximally two variants, $K_{i, A}^{+}$and $K_{i, A}^{-}$. Similarly, each $K_{i, B}$ has maximally four variants, $K_{i, B}^{++}, K_{i, B}^{+-}, K_{i, B}^{-+}$and $K_{i, B}^{--}$. In total, there are maximally six variants of $K_{i}$ for each information transfer. Since we study 28 information transfers, there are maximally 168 possible $K_{i}, 168$ possible $\Gamma_{i}^{j}$ and 168 possible $\Gamma_{i}^{k l}$. In order to keep this article at a reasonable length, we use a very condensed way to express all these, in total, maximally 504 quantities. 
7.9 Zero average force on particle $A$

Information transfers $I_{1}, I_{2}, I_{3}, I_{9}, I_{10}$ and $I_{11}$ satisfy $\tau_{0}=\tau_{A}$ and $S=A$. Let $z$-axis vector be defined as

$$
\mathbf{z}=\mathbf{x}_{B}\left(\tau_{B}\right)-\mathbf{x}_{A}\left(\tau_{A}\right)
$$

Mediatorino $m$ is at time $\tau_{A}$ co-local with particle $A$. For point-like particle $A$, it means that $\triangle \mathbf{q}$ is always determined by law 1-(a), that is the distribution $K_{i, A}^{+}$is the same for all directions, $K_{i, A}^{+}=$const. Using the condition (34), we get that for all $i \in\{1,2,3,9,10,11\}$

$$
K_{i, A}^{+}(\hat{\mathbf{e}})=1 / 4 \pi
$$

We use this value to express the 1-rank tensor $\Gamma_{i, A}^{j+}$ and 2-rank tensor $\Gamma_{i, A}^{k l+}$ by equation (37). Namely,

$$
\begin{aligned}
\Gamma_{i, A}^{j+} & =(0,0,0)^{T}, \\
\Gamma_{i, A}^{k l+} & =\left(\begin{array}{ccc}
1 / 3 & 0 & 0 \\
0 & 1 / 3 & 0 \\
0 & 0 & 1 / 3
\end{array}\right)
\end{aligned}
$$

The former equation implies that the mean force $\left\langle\mathbf{F}_{i, A}^{+}\right\rangle$on particle $A$ is zero,

$$
\left\langle\mathbf{F}_{i, A}^{+}\right\rangle=\mathbf{0},
$$

while the latter equation implies that the mean dispersion tensor on particle $A$ is spherically symmetric. In other words, the studied information transfers tend to disperse particle $A$ equally in all the directions.

\subsection{Constant force on particle $A$}

Information transfers $I_{17}, I_{18}, I_{19}, I_{25}, I_{26}$ and $I_{27}$ satisfy $\tau_{0}=\tau_{A}$ and $S=B$. Let $z$-axis vector be defined as

$$
\mathbf{z}=\mathbf{x}_{B}\left(\tau_{A}\right)-\mathbf{x}_{A}\left(\tau_{A}\right)
$$

Mediatorino $m$ is at time $\tau_{A}$ still on particle $B$, and thus is generally non-co-local with particle $A$. It means that $\triangle \mathbf{q}$ is always determined by law 1-(b), that is in the direction of particle $B$ from particle $A$. Since particles $A$ and $B$ are at time $\tau_{A}$ both point-like, the directional distribution $K_{i, A}$ is non-zero only for one direction represented by angles $\vartheta_{0}$ and $\varphi_{0}$. Let $\delta_{d}(\vartheta, \varphi)$ be a directional Dirac delta function defined in the way that for every ordinary function $g(\vartheta, \varphi)$ and every $\vartheta_{0} \in[0, \pi], \varphi_{0} \in[0,2 \pi]$,

$$
\oint_{\vartheta, \varphi} g(\vartheta, \varphi) \delta_{d}\left(\vartheta-\vartheta_{0}, \varphi-\varphi_{0}\right) \sin \vartheta d \vartheta d \varphi=g\left(\vartheta_{0}, \varphi_{0}\right) .
$$

Using the condition in equation (34), we get that $K_{i, A}$ can be expressed as

$$
K_{i, A}(\vartheta, \varphi)=g(\vartheta, \varphi) \cdot \delta_{d}\left(\vartheta-\vartheta_{0}, \varphi-\vartheta_{0}\right),
$$

where $g$ is an unknown function satisfying $g\left(\vartheta_{0}, \varphi_{0}\right)=1$. Without loss of generality, we can choose the simplest form of $K_{i, A}$ where for all $\vartheta, \varphi: g(\vartheta, \varphi)=1$. Moreover, for distribution 
$K_{i, A}^{+}$, there is $\vartheta_{0}=\pi$. In this polar situation $\varphi_{0}$ is irrelevant, so we choose $\varphi_{0}=\pi$. Thus, for all $i \in\{17,18,19,25,26,27\}$ we have

$$
K_{i, A}^{+}(\vartheta, \varphi)=\delta_{d}(\vartheta-\pi, \varphi-\pi) .
$$

Then, we can easily calculate the 1-rank tensor $\Gamma_{i, A}^{j+}$ and 2-rank tensor $\Gamma_{i, A}^{k l+}$,

$$
\begin{aligned}
\Gamma_{i, A}^{j+} & =(0,0,-1)^{T}, \\
\Gamma_{i, A}^{k l+} & =\left(\begin{array}{lll}
0 & 0 & 0 \\
0 & 0 & 0 \\
0 & 0 & 1
\end{array}\right) .
\end{aligned}
$$

This leads to the fact that the mean force $\left\langle\mathbf{F}_{i, A}^{+}\right\rangle$on particle $A$ is constant and points in the direction of $-\mathbf{z}$-axis,

$$
\left\langle\mathbf{F}_{i, A}^{+}\right\rangle=-f_{i, A} \cdot \hat{\mathbf{z}}
$$

and that the average dispersion tensor $\left\langle D_{i, A}^{+}\right\rangle$does not tend to disperse particle $A$ in all the directions but in only one.

\subsection{Intermezzo for transfers with $\tau_{0}<\tau_{A}<\tau_{E}$}

In the transfers with $\tau_{0}<\tau_{A}<\tau_{E}$ mediatorino $m$ is at time $\tau_{A}$ on a sphere. It is a consequence of the fact that particle $S$ is at time $\tau_{0}$ a point-like particle, the speed of mediatorino $m$ is during time interval $\left(\tau_{0}, \tau_{A}\right)$ constant and positive, $v_{m}\left(\tau_{0 A}\right)>0$, and that $\tau_{A}-\tau_{0}$ is constant. The sphere (hereafter denoted by $\left.\mathscr{S}_{R}\right)$ has its center at $\mathbf{x}_{S}\left(\tau_{0}\right)$ and radius

$$
R=v_{m}\left(\tau_{0 A}\right) \cdot\left(\tau_{A}-\tau_{0}\right) .
$$

Unlike the mediatorino, particle $A$ can at time $\tau_{A}$ be only at single point $\mathbf{x}_{A}\left(\tau_{A}\right)$. This leads us to a sphere-to-point situation (denoted by $s p$ ) which is axis-symmetric with respect to

$$
\mathbf{z}=\mathbf{r}_{S}=\mathbf{x}_{S}\left(\tau_{0}\right)-\mathbf{x}_{A}\left(\tau_{A}\right),
$$

where $\mathbf{z}$ is the $z$-axis vector and $\mathbf{r}_{S}$ is a vector with characteristic length $r_{S}$.

\subsubsection{Directional distribution}

Let us examine the directional distribution of the sphere-to-point situation, $K_{s p, A}^{+}$. Since $\mathbf{x}_{m}^{c}\left(\tau_{A}\right)$ is at time $\tau_{A}$ generally not co-local with particle $A$, direction $\triangle \hat{\mathbf{q}}$ of $\triangle \mathbf{q}$ is determined by law 1-(b). If particle $A$ is at time $\tau_{A}$ inside sphere $\mathscr{S}_{R}, r_{S}<R$, then every direction $\triangle \hat{\mathbf{q}}$ (when its origin is moved to $\mathbf{x}_{A}\left(\tau_{A}\right)$ ) intersects the sphere exactly once. It means that $K_{s p, A}^{+}\left(\mathbf{r}_{S}, \hat{\mathbf{e}}\right)$ is composed of only one contribution corresponding to the unique $\mathbf{x}_{m}^{c}\left(\tau_{A}\right)$ lying on the sphere in the direction $\triangle \hat{\mathbf{q}}$ from $\mathbf{x}_{A}\left(\tau_{A}\right)$. On the other hand, if particle $A$ is outside the sphere or on its surface, $r_{S} \geq R$, then direction $\triangle \hat{\mathbf{q}}$ (when its origin is moved to $\mathbf{x}_{A}\left(\tau_{A}\right)$ ) can intersect the sphere twice, once, or zero times depending on the value of $\vartheta$. It can be rephrased in the way that for a given direction $\triangle \hat{\mathbf{q}}, K_{s p, A}^{+}\left(\mathbf{r}_{S}, \hat{\mathbf{e}}\right)$ can be composed of two, one, or zero contributions, each corresponding to a different $\mathbf{x}_{m}^{c}\left(\tau_{A}\right)$ lying on the sphere in the direction $\triangle \hat{\mathbf{q}}$ from $\mathbf{x}_{A}\left(\tau_{A}\right)$. Since the intersection of direction $\triangle \hat{\mathbf{q}}$ and sphere $\mathscr{S}_{R}$ contributes to $K_{s p, A}^{+}\left(\mathbf{r}_{S}, \hat{\mathbf{e}}\right)$ only when $\triangle \hat{\mathbf{q}}=-\hat{\mathbf{e}}$, distribution $K_{s p, A}^{+}\left(\mathbf{r}_{S}, \hat{\mathbf{e}}\right)$ can be expressed as 


$$
K_{s p, A}^{+}\left(\mathbf{r}_{S}, \hat{\mathbf{e}}\right)= \begin{cases}\left|k_{+}(-\hat{\mathbf{e}})\right|, & \text { if } r_{S}<R, \vartheta \in[0, \pi], \\ 0, & \text { if } r_{S} \geq R, \vartheta \in\left[0, \pi-\vartheta_{R}\right), \\ \left|k_{+}(-\hat{\mathbf{e}})\right|, & \text { if } r_{S} \geq R, \vartheta=\pi-\vartheta_{R}, \\ \left|k_{+}(-\hat{\mathbf{e}})\right|+\left|k_{-}(-\hat{\mathbf{e}})\right|, & \text { if } r_{S} \geq R, \vartheta \in\left(\pi-\vartheta_{R}, \pi\right],\end{cases}
$$

where $\hat{\mathbf{e}}=\hat{\mathbf{e}}(\vartheta, \varphi), k_{+}(-\hat{\mathbf{e}})$ and $k_{-}(-\hat{\mathbf{e}})$ are the individual contributions and

$$
\vartheta_{R}=\arcsin \left(\frac{R}{r_{S}}\right)
$$

The absolute values were added to equation (70) to ensure that all the contributions sum constructively.

Let us look at how $k(\hat{\mathbf{e}})$, a contribution to directional distribution $K_{s p, A}^{+}\left(\mathbf{r}_{S},-\hat{\mathbf{e}}\right)$, is transformed. We use primes when we want to describe a special situation where particle $A$ is at time $\tau_{A}$ at the center of the sphere, $\mathbf{x}_{A}\left(\tau_{A}\right)=\mathbf{x}_{S}\left(\tau_{0}\right)$. Let us assume every $\mathbf{x}_{m}^{c}\left(\tau_{A}\right)$ on the surface of the sphere $\mathscr{S}_{R}$ has the same probability $d P^{\prime}=k^{\prime} d \Omega^{\prime}$ of being chosen. It means that $k^{\prime}$ is constant. Moreover, once we require that the sum of probability contributions $d P^{\prime}$ on the whole sphere equals one, we get $k^{\prime}=1 / 4 \pi$. Since every choice of $\mathbf{x}_{m}^{c}\left(\tau_{A}\right)$ is followed by taking $\triangle \mathbf{q}$ from particle $A$, the probability $d P^{\prime}$ fully contributes to $d P=k d \Omega$. Thus, we have $k^{\prime} d \Omega^{\prime}=k(\hat{\mathbf{e}}) d \Omega$, or equivalently

$$
k^{\prime} \sin \vartheta^{\prime} d \vartheta^{\prime} d \varphi^{\prime}=k(\hat{\mathbf{e}}) \sin \vartheta d \vartheta d \varphi .
$$

There is $d \varphi^{\prime}=d \varphi$. If we use equation (24) and define

$$
\mathbf{l}^{\prime}=\mathbf{x}_{m}^{c}\left(\tau_{A}\right)-\mathbf{x}_{S}\left(\tau_{0}\right)
$$

then $l^{\prime} \sin \vartheta^{\prime}=l \sin \vartheta$. Moreover, $l d \vartheta=l^{\prime} d \vartheta^{\prime} \cdot \cos \omega$, where $\omega$ is the angle between vector $\mathbf{I}$ and vector $\mathbf{I}^{\prime}$. Since angle $\omega$ satisfies $\cos \omega=(l-r \cos \vartheta) / l^{\prime}$, we get

$$
k(\hat{\mathbf{e}})=k^{\prime} \cdot \frac{l^{2}}{l^{\prime}\left(l-r_{S} \cos \vartheta\right)} .
$$

The value of $l$ can be evaluated from the law of cosines and with use of $l^{\prime}=R$ we obtain

$$
l_{ \pm}=r_{S} \cos \vartheta \pm \sqrt{R^{2}-r_{S}^{2} \sin ^{2} \vartheta}
$$

The location of mediatorino $m$ at time $\tau_{A}$, then, can be expressed as

$$
\mathbf{x}_{m \pm}^{c}\left(\tau_{A}, \vartheta, \varphi\right)=\mathbf{x}_{A}\left(\tau_{A}\right)+l_{ \pm} \cdot \hat{\mathbf{e}}(\vartheta, \varphi)
$$

When we express $k$ explicitly using $k^{\prime}=1 / 4 \pi$, we obtain

$$
k_{ \pm}(\hat{\mathbf{e}})= \pm \frac{\left(r_{S} \cos \vartheta \pm \sqrt{R^{2}-r_{S}^{2} \sin ^{2} \vartheta}\right)^{2}}{4 \pi R \cdot \sqrt{R^{2}-r_{S}^{2} \sin ^{2} \vartheta}} .
$$


While for $r_{S}<R$ only $k_{+}$is usable, for $r_{S} \geq R$ both $k_{+}$and $k_{-}$are usable. Now we can combine equations (70) and (77) in order to explicitly express $K_{s p, A}^{+}\left(\mathbf{r}_{S}, \hat{\mathbf{e}}\right)$,

$$
K_{s p, A}^{+}\left(\mathbf{r}_{S}, \hat{\mathbf{e}}\right)= \begin{cases}\frac{\left(-r_{S} \cos \vartheta+\sqrt{R^{2}-r_{S}^{2} \sin ^{2} \vartheta}\right)^{2}}{4 \pi R \cdot \sqrt{R^{2}-r_{S}^{2} \sin ^{2} \vartheta},} & \text { if } r_{S}<R, \vartheta \in[0, \pi], \\ 0, \quad & \text { if } r_{S} \geq R, \vartheta \in\left[0, \pi-\vartheta_{R}\right), \\ \frac{R^{2}+r_{S}^{2} \cos 2 \vartheta}{2 \pi R \cdot \sqrt{R^{2}-r_{S}^{2} \sin ^{2} \vartheta},} & \text { if } r_{S} \geq R, \vartheta \in\left(\pi-\vartheta_{R}, \pi\right],\end{cases}
$$

where we excluded the infinite value of $K_{s p, A}^{+}$for $r \geq R, \vartheta=\pi-\vartheta_{R}$. This redefinition does not influence $\Gamma_{s p, A}^{j+}$ and $\Gamma_{s p, A}^{k l+}$ based on an integration of $K_{s p, A}^{+}$. While $\Gamma_{s p, A}^{3+}$ can be expressed as

$$
\Gamma_{s p, A}^{3+}\left(\mathbf{r}_{S}\right)= \begin{cases}-\frac{2 r_{S}}{3 R}, & \text { if } r_{S} \leq R, \\ -\left(1-\frac{R^{2}}{3 r_{S}^{2}}\right), & \text { if } r_{S} \geq R,\end{cases}
$$

$\Gamma_{s p, A}^{11+}$ and $\Gamma_{s p, A}^{22+}$ can be expressed as

$$
\Gamma_{s p, A}^{11+}\left(\mathbf{r}_{S}\right)=\Gamma_{s p, A}^{22+}\left(\mathbf{r}_{S}\right)= \begin{cases}1 / 3, & \text { if } r_{S}=0, \\ \frac{1}{8}+\frac{R^{2}}{8 r_{S}^{2}}+\frac{\left(R^{2}-r_{S}^{2}\right)^{2}}{16 R r_{S}^{3}} \ln \left(\frac{R-r_{S}}{R+r_{S}}\right), & \text { if } 0<r_{S}<R, \\ 1 / 4, & \text { if } r_{S}=R, \\ \frac{1}{8}+\frac{R^{2}}{8 r_{S}^{2}}+\frac{\left(r_{S}^{2}-R^{2}\right)^{2}}{16 R r_{S}^{3}} \ln \left(\frac{r_{S}-R}{r_{S}+R}\right), & \text { if } r_{S}>R,\end{cases}
$$

and finally $\Gamma_{s p, A}^{33+}$ can be expressed as

$$
\Gamma_{s p, A}^{33+}\left(\mathbf{r}_{S}\right)= \begin{cases}1 / 3, & \text { if } r_{S}=0, \\ \frac{3}{4}-\frac{R^{2}}{4 r_{S}^{2}}-\frac{\left(R^{2}-r_{S}^{2}\right)^{2}}{8 R r_{S}^{3}} \ln \left(\frac{R-r_{S}}{R+r_{S}}\right), & \text { if } 0<r_{S}<R, \\ 1 / 2, & \text { if } r_{S}=R \\ \frac{3}{4}-\frac{R^{2}}{4 r_{S}^{2}}-\frac{\left(r_{S}^{2}-R^{2}\right)^{2}}{8 R r_{S}^{3}} \ln \left(\frac{r_{S}-R}{r_{S}+R}\right), & \text { if } r_{S}>R\end{cases}
$$

\subsubsection{Restriction of movement of mediatorino $m$}

Mediatorinos do not necessarily move in full space. In the transfers with $\tau_{0}<\tau_{A}<\tau_{E}$ if $r_{S}>R$, then the movement of the mediatorinos within time interval $\left(\tau_{A}, \tau_{B}\right)$ is bounded by a double cone. Let $\mathscr{C}^{+}(\mathbf{x}, \mathbf{r}, \rho)$ be a cone defined by the apex at point $\mathbf{x}$, the axis parallel to line segment $\mathbf{r}$, and the sphere with the center at $\mathbf{x}+\mathbf{r}$ and radius $\rho$ that is inscribed to the cone. Let also $\mathscr{C}^{-}(\mathbf{x}, \mathbf{r}, \rho)=\mathscr{C}^{+}(\mathbf{x},-\mathbf{r}, \rho)$ and $\mathscr{C}(\mathbf{x}, \mathbf{r}, \rho)=\mathscr{C}^{+}(\mathbf{x}, \mathbf{r}, \rho) \cup \mathscr{C}^{-}(\mathbf{x}, \mathbf{r}, \rho)$. Finally, the set of all the unit vectors which originate at point $\mathbf{x}$ and are within $\mathscr{C}^{+}, \mathscr{C}^{-}$and $\mathscr{C}$ will be denoted by $E^{+}, E^{-}$, and $E$, respectively. Namely, 


$$
\begin{aligned}
E^{ \pm}(\mathbf{x}, \mathbf{r}, \rho) & =\left\{\hat{\mathbf{e}} \mid \mathbf{x}+\hat{\mathbf{e}} \in \mathscr{C}^{ \pm}(\mathbf{x}, \mathbf{r}, \rho)\right\}, \\
E(\mathbf{x}, \mathbf{r}, \rho) & =\{\hat{\mathbf{e}} \mid \mathbf{x}+\hat{\mathbf{e}} \in \mathscr{C}(\mathbf{x}, \mathbf{r}, \rho)\} .
\end{aligned}
$$

If we do not consider the sign of $s_{q}$, then all the possible $\triangle \mathbf{q}$ (when their origin is moved to $\left.\mathbf{x}_{A}\left(\tau_{A}\right)\right)$ are within double cone $\mathscr{C}_{R}=\mathscr{C}\left(\mathbf{x}_{A}\left(\tau_{A}\right), \mathbf{r}_{S}, R\right)$. The apex angle of $\mathscr{C}_{R}$ is $2 \vartheta_{R}$, where $\vartheta_{R}$ is defined by equation (71). Finally, the set of all the possible unit vectors $\triangle \hat{\mathbf{q}}$ is $E_{R}=E\left(\mathbf{x}_{A}\left(\tau_{A}\right), \mathbf{r}_{S}, R\right)$. Since $\mathbf{v}_{A}\left(\tau_{A B}\right)$ is either parallel or antiparallel to $\triangle \mathbf{q}$, and since $m$ is at time $\tau_{A}$ on the surface of sphere $\mathscr{S}_{R}$ that is inscribed to the double cone, we conclude that in the transfers with $\tau_{0}<\tau_{A}<\tau_{E}$ during $\left(\tau_{A}, \tau_{B}\right)$ all the mediatorinos move inside or on the surface of double cone $\mathscr{C}_{R}$. Double cone $\mathscr{C}_{R}$ can be decomposed into two cones, $\mathscr{C}_{R}^{-}$ and $\mathscr{C}_{R}^{+}$, with common point $\mathbf{x}_{A}\left(\tau_{A}\right)$. The cone $\mathscr{C}_{R}^{+}$itself is composed of three disjunctive parts

$$
\mathscr{C}_{R}^{+}=\mathscr{C}_{R, c}^{+} \cup \mathscr{S}_{R} \cup \mathscr{C}_{R, f}^{+},
$$

where $\mathscr{S}_{R}$ is the already mentioned sphere with center at $\mathbf{x}_{A}\left(\tau_{A}\right)+\mathbf{r}_{S}$ and radius $R . \mathscr{C}_{R, c}^{+}$ is the set of the points of $\mathscr{C}_{R}^{+}$that are closer to point $\mathbf{x}_{A}\left(\tau_{A}\right)$ than sphere $\mathscr{S}_{R}$, and finally $\mathscr{C}_{R, f}^{+}$is the set of the points of $\mathscr{C}_{R}^{+}$that are farther from $\mathbf{x}_{A}\left(\tau_{A}\right)$ than sphere $\mathscr{S}_{R}$. Note that for $r_{S} \leq R, \mathscr{C}_{R}$ is no longer a double cone, but rather full space. It means that, in that case, the mediatorinos can during time interval $\left(\tau_{A}, \tau_{B}\right)$ move in any direction. Moreover, space is divided into two relevant parts $\mathscr{S}_{R}$ and $\mathbb{R}^{3} \backslash \mathscr{S}_{R}$.

\subsection{Asymptotically free force on particle $A$}

Information transfers $I_{21}, I_{22}, I_{23}, I_{29}, I_{30}$ and $I_{31}$ satisfy $\tau_{0}<\tau_{A}<\tau_{E}$ and $S=B$. Let vectors $\mathbf{z}$ and $\mathbf{r}_{S}$ be defined as

$$
\mathbf{z}=\mathbf{r}_{S}=\mathbf{x}_{B}\left(\tau_{0}\right)-\mathbf{x}_{A}\left(\tau_{A}\right) .
$$

Mediatorino $m$ is at time $\tau_{A}$ on sphere $\mathscr{S}_{R}$, that is on a sphere with center at $\mathbf{x}_{B}\left(\tau_{0}\right)$ and radius $R=v_{m}\left(\tau_{0 A}\right) \cdot\left(\tau_{A}-\tau_{0}\right)$, where $v_{m}\left(\tau_{0 A}\right)>0$ is the speed of the mediatorino during $\left(\tau_{0}, \tau_{A}\right)$. The directional distribution $K_{i, A}^{+}$can, then, be expressed for all $i \in\{21,22,23,29,30,31\}$ as

$$
K_{i, A}^{+}\left(\mathbf{r}_{S}, \hat{\mathbf{e}}\right)=K_{s p, A}^{+}\left(\mathbf{r}_{S}, \hat{\mathbf{e}}\right) .
$$

When we calculate the mean force, we get

$$
\left\langle\mathbf{F}_{i, A}^{+}\left(\mathbf{r}_{S}\right)\right\rangle= \begin{cases}-f_{i, A} \cdot \frac{2 r_{S}}{3 R} \cdot \hat{\mathbf{z}}, & \text { if } r_{S} \leq R, \\ -f_{i, A} \cdot\left(1-\frac{R^{2}}{3 r_{S}^{2}}\right) \cdot \hat{\mathbf{z}}, & \text { if } r_{S} \geq R .\end{cases}
$$

Since the force depends on the distance of particles $A$ and $B$ and satisfies

$$
\begin{aligned}
\lim _{r_{S} \rightarrow 0}\left\langle\mathbf{F}_{i, A}^{+}\left(r_{S} \cdot \hat{\mathbf{z}}\right)\right\rangle=\mathbf{0}, \\
\lim _{r_{S} \rightarrow \infty}\left\langle\mathbf{F}_{i, A}^{+}\left(r_{S} \cdot \hat{\mathbf{z}}\right)\right\rangle=-f_{i, A} \cdot \hat{\mathbf{z}},
\end{aligned}
$$

we are justified in calling these transfers asymptotically free forces. If we knew the transfers are attractive at large distances, we would call them asymptotically free confining forces. 
7.13 Speed-dependent force on particle $A$

Information transfers $I_{5}, I_{6}, I_{7}, I_{13}, I_{14}$ and $I_{15}$ satisfy $\tau_{0}<\tau_{A}<\tau_{E}$ and $S=A$. Let vectors $\mathbf{z}$ and $\mathbf{r}_{S}$ be defined as

$$
\mathbf{z}=\mathbf{r}_{S}=\mathbf{x}_{A}\left(\tau_{0}\right)-\mathbf{x}_{A}\left(\tau_{A}\right)
$$

Mediatorino $m$ is at time $\tau_{A}$ on sphere $\mathscr{S}_{R}$, that is on a sphere with center at $\mathbf{x}_{A}\left(\tau_{0}\right)$ and radius $R=v_{m}\left(\tau_{0 A}\right) \cdot\left(\tau_{A}-\tau_{0}\right)$, where $v_{m}\left(\tau_{0 A}\right)>0$ is the speed of the mediatorino. The directional distribution $K_{i, A}^{+}$can be expressed for all $i \in\{5,6,7,13,14,15\}$ as

$$
K_{i, A}^{+}\left(\mathbf{r}_{S}, \hat{\mathbf{e}}\right)=K_{s p, A}^{+}\left(\mathbf{r}_{S}, \hat{\mathbf{e}}\right) .
$$

Once we express $r_{S}=v_{A}\left(\tau_{0 A}\right) \cdot\left(\tau_{A}-\tau_{0}\right)$, where $v_{A}\left(\tau_{0 A}\right) \geq 0$, we see that $K_{i, A}^{+}$does not depend on the distance of particles $A$ and $B$ and depends on the speeds $v_{m}\left(\tau_{0 A}\right)$ and $v_{A}\left(\tau_{0 A}\right)$. For the mean force we get

$$
\left\langle\mathbf{F}_{i, A}^{+}\left(\mathbf{r}_{S}\right)\right\rangle= \begin{cases}-f_{i, A} \cdot \frac{2 v_{A}}{3 v_{m}} \cdot \hat{\mathbf{z}}, & \text { if } v_{A} \leq v_{m}, \\ -f_{i, A} \cdot\left(1-\frac{v_{m}^{2}}{3 v_{A}^{2}}\right) \cdot \hat{\mathbf{z}}, & \text { if } v_{A} \geq v_{m},\end{cases}
$$

which naturally also depends on speeds $v_{m}\left(\tau_{0 A}\right)$ and $v_{A}\left(\tau_{0 A}\right)$, rather than on the distance of particles $A$ and $B$.

\subsection{Implementation of co-locality}

The information transfers with $\tau_{B}=\tau_{E}$, in order to transfer $\Delta q$ to particle $B$, require mediatorino $m$ to be co-local with particle $T$. That is, the mediatorino must hit target particle $T$. Since there is a zero probability that point-like mediatorino $m$ hits point-like particle $T$ in continuous space of dimension $d>1$, it raises the question what co-local really means and how it is implemented. There are several ways to get a non-zero probability, and make the information transfers work: (a) space is of dimension $d=0$ or $d=1$, or (b) space is discrete, not continuous, or (c) mediatorino $m$ and/or particle $T$ is (almost) never a point-like particle or at least it works as if it had a non-zero interaction size. From experience we know that space is neither of dimension zero nor of dimension one. Moreover, there has been no evidence for the discreteness of space so far. We have also assumed that particle $T$ is at time $\tau_{E}$ a point-like particle. The remaining possibility is that mediatorino $m$ has an interaction size. From now on, we assume mediatorino $m$ behaves as if it were a sphere with center at $\mathbf{x}_{m}(t)$ and a relatively small radius $w>0$. In other words, in the transfers with $\tau_{B}=\tau_{E}$ mediatorino $m$ can hit target particle $T$ if the distance of $\mathbf{x}_{m}(t)$ from particle $T$ becomes at some moment no greater than $w$. The cross section of the mediatorino is, therefore, of order $w^{2}$. If mediatorino $m$ starts on particle $S$, then the probability of hitting particle $T$ randomly located in 3-dimensional space can be estimated as

$$
P(r) \propto w^{2} / r^{2},
$$

where $r$ is the distance between particle $S$ and particle $T$. In total, in the transfers with $\tau_{B}=\tau_{E}$ the influence between two particles rapidly decreases as their distance increases. 
7.15 Force on particle $B$ in transfers with $\tau_{B}<\tau_{E}$

The information transfers with $\tau_{B}<\tau_{E}$ transfer $\Delta q$ at time $\tau_{B}$ to particle $B$ via one or two entanglements. Unlike the transfers with $\tau_{B}=\tau_{E}$, the transfers with $\tau_{B}<\tau_{E}$ in order to transfer $\Delta q$ to particle $B$ do not require mediatorino $m$ to be co-local with particle $T$. On the other hand, there may be a requirement for the non-co-locality of mediatorino $m$ with particle $T$. This requirement is in 3-dimensional space very weak and is almost always satisfied. Even if mediatorino $m$ and particle $T$ were co-local with each other, the mediatorino can wait quite a short time until the requirement is satisfied, and then it can use the entanglement(s) to transfer $\Delta q$ to particle $B$. In other words, if $m$ starts and takes some $\Delta q$ from particle $A$, then there is probability

$$
P(r)=1,
$$

that $\triangle q$ will eventually be transferred to particle $B$, no matter what distance $r$ between particles $S$ and $T$ is, and no matter what the dimension of the space is. The transfers with $\tau_{B}<\tau_{E}$ are, therefore, maximally effective. For every odd $i \in \mathbb{F}$, we can immediately write

$$
\begin{aligned}
\mathbf{r}_{T} & =\mathbf{r}_{S}, \\
K_{i, B}^{+ \pm}\left(\mathbf{r}_{T}, \hat{\mathbf{e}}\right) & =K_{i, A}^{+}\left(\mathbf{r}_{S},-\hat{\mathbf{e}}\right), \\
\Gamma_{i, B}^{j+ \pm}\left(\mathbf{r}_{T}\right) & =-\Gamma_{i, A}^{j+}\left(\mathbf{r}_{S}\right), \\
\Gamma_{i, B}^{k l+ \pm}\left(\mathbf{r}_{T}\right) & =\Gamma_{i, A}^{k l+}\left(\mathbf{r}_{S}\right), \\
\left\langle\mathbf{F}_{i, B}\left(\mathbf{r}_{T}\right)\right\rangle & =-\left\langle\mathbf{F}_{i, A}\left(\mathbf{r}_{S}\right)\right\rangle .
\end{aligned}
$$

It means that the transfers with $\tau_{B}<\tau_{E}$ are completely described, and from now on we look for $K_{i}, \Gamma_{i}^{j}$, and $\Gamma_{i}^{k l}$ only within the transfers with $\tau_{B}=\tau_{E}$.

\subsection{Hitting particle $T$}

Here we examine hitting particle $T$ by a mediatorino in transfers $I_{2}, I_{6}, I_{10}, I_{14}, I_{18}, I_{22}$, $I_{26}$, and $I_{30}$ satisfying $\tau_{A}<\tau_{B}=\tau_{E}$. If mediatorino $m$ is a sphere with center at $\mathbf{x}_{m}(t)$ and radius $w>0$, then hitting point-like particle $T$ at time $\tau_{E}$ for mediatorino $m$ means

$$
\left|\mathbf{x}_{m}\left(\tau_{E}\right)-\mathbf{x}_{T}\left(\tau_{E}\right)\right| \leq w .
$$

It can be rephrased in the way that $\mathbf{x}_{m}\left(\tau_{E}\right)$ must intersect a sphere (hereafter denoted by $\left.\mathscr{S}_{w}\right)$ with center at $\mathbf{x}_{T}\left(\tau_{E}\right)$ and radius $w$. Once during a single transfer $I_{i}$ mediatorino $m$ hits particle $T, K_{i, A}\left(\mathbf{r}_{S},-\hat{\mathbf{e}}\right)$ with a specific $\hat{\mathbf{e}}$ contributes to $K_{i, B}\left(\mathbf{r}_{T}, \hat{\mathbf{e}}\right)$. That is, for information transfers $I_{i}$ with $i \in\{2,6,10,14,18,22,26,30\}$ :

$$
K_{i, B}\left(\mathbf{r}_{T}, \hat{\mathbf{e}}\right)= \begin{cases}K_{i, A}\left(\mathbf{r}_{S},-\hat{\mathbf{e}}\right), & \text { if } m \text { hits } T, \\ 0, & \text { otherwise }\end{cases}
$$

where $\mathbf{r}_{T}$ and its direction $\hat{\mathbf{r}}_{T}$ are defined as

$$
\begin{aligned}
& \mathbf{r}_{T}=r_{T} \cdot \hat{\mathbf{r}}_{T}=\mathbf{x}_{T}\left(\tau_{E}\right)-\mathbf{x}_{A}\left(\tau_{A}\right), \\
& \hat{\mathbf{r}}_{T}=\left(\sin \vartheta_{T} \cos \varphi_{T}, \sin \vartheta_{T} \sin \varphi_{T}, \cos \vartheta_{T}\right) .
\end{aligned}
$$

Since sphere $S_{w}$ is of a limited size, in most cases not every location $\mathbf{x}_{m}\left(\tau_{A}\right)$ and not every direction of $\mathbf{v}_{m}\left(\tau_{A B}\right)$ allow mediatorino $m$ to hit particle $T$. Hence, the condition in equation (101) that the mediatorino hits particle $T$ means that a condition on $\mathbf{x}_{m}\left(\tau_{A}\right)$ and a 
condition on $\mathbf{v}_{m}\left(\tau_{A B}\right)$ must be satisfied. The velocity $\mathbf{v}_{m}\left(\tau_{A B}\right)$ with direction $\hat{\mathbf{v}}_{m}$ is either parallel or antiparallel to $\triangle \hat{\mathbf{q}}$ which itself is in $K_{i, B}\left(\mathbf{r}_{T}, \hat{\mathbf{e}}\right)$ represented by $+\hat{\mathbf{e}}$. Therefore, the condition on $\mathbf{v}_{m}\left(\tau_{A B}\right)$ will usually be expressed as the corresponding condition on $\hat{\mathbf{e}}$.

Let us start with a study of hitting particle $T$ in the case of $r_{T} \leq w$, that is when particle $A$ is at time $\tau_{A}$ in sphere $\mathscr{S}_{w}$. If velocity $\mathbf{v}_{m}\left(\tau_{A B}\right)$ points away from particle $A, s_{v}=+1$, then the mediatorino can hit particle $T$ only when $\mathbf{x}_{m}\left(\tau_{A}\right)$ is in the sphere. On the other hand, if velocity $\mathbf{v}_{m}\left(\tau_{A B}\right)$ points toward particle $A, s_{v}=-1$, the mediatorino can hit particle $T$ always. We get

$$
\begin{aligned}
& K_{i, B}^{++}\left(\mathbf{r}_{T}, \hat{\mathbf{e}}\right)= \begin{cases}K_{i, A}^{+}\left(\mathbf{r}_{S},-\hat{\mathbf{e}}\right), & \text { if } \mathbf{x}_{m}\left(\tau_{A}\right) \in \mathscr{S}_{w}, \\
0, & \text { otherwise, }\end{cases} \\
& K_{i, B}^{+-}\left(\mathbf{r}_{T}, \hat{\mathbf{e}}\right)=K_{i, A}^{+}\left(\mathbf{r}_{S},-\hat{\mathbf{e}}\right) .
\end{aligned}
$$

Now we move to the case of $r_{T}>w$, that is when particle $A$ is at time $\tau_{A}$ outside sphere $\mathscr{S}_{w}$. Let $\mathscr{C}_{w}=\mathscr{C}\left(\mathbf{x}_{A}\left(\tau_{A}\right), \mathbf{r}_{T}, w\right)$ be a double cone composed of two cones, $\mathscr{C}_{w}^{-}$and $\mathscr{C}_{w}^{+}$, having a common point $\mathbf{x}_{A}\left(\tau_{A}\right)$. Moreover, let $\mathscr{C}_{w}^{+}$be composed of three disjunctive parts,

$$
\mathscr{C}_{w}^{+}=\mathscr{C}_{w, c}^{+} \cup \mathscr{S}_{w} \cup \mathscr{C}_{w, f}^{+},
$$

where $\mathscr{S}_{w}$ is the already mentioned sphere with center at $\mathbf{x}_{T}\left(\tau_{E}\right)$ and radius $w, \mathscr{C}_{w, c}^{+}$is $\mathbf{x}_{A}\left(\tau_{A}\right)$ plus the set of all the points of $\mathscr{C}_{w}^{+}$that are between point $\mathbf{x}_{A}\left(\tau_{A}\right)$ and sphere $\mathscr{S}_{w}$, and $\mathscr{C}_{w, f}^{+}$is the set of all the points of $\mathscr{C}_{w}^{+}$that are from point $\mathbf{x}_{A}\left(\tau_{A}\right)$ farther than sphere $\mathscr{S}_{w}$. The apex angle of each of cones $\mathscr{C}_{w}^{+}$and $\mathscr{C}_{w}^{-}$is $2 \vartheta_{w}$ where

$$
\vartheta_{w}\left(\mathbf{r}_{T}\right)=\arccos \left(\sqrt{1-\frac{w^{2}}{r_{T}^{2}}}\right) .
$$

The corresponding apex solid angle $\Omega_{w}$ can be calculated as $\Omega_{w}=2 \pi\left(1-\cos \vartheta_{w}\right)$, and thus

$$
\Omega_{w}\left(\mathbf{r}_{T}\right)=2 \pi \cdot\left(1-\sqrt{1-\frac{w^{2}}{r_{T}^{2}}}\right) .
$$

Since mediatorino $m$ moves during $\left(\tau_{A}, \tau_{B}\right)$ along line $\mathbf{I}$ defined by equation (24), the mediatorino can hit particle $T$ only when $\mathbf{x}_{m}\left(\tau_{A}\right)$ is on the surface or inside double cone $\mathscr{C}_{w}$, that is when $\hat{\mathbf{e}} \in E_{w}$. Moreover, velocity $\mathbf{v}_{m}\left(\tau_{A B}\right)$ must have a good orientation. Namely, if velocity $\mathbf{v}_{m}\left(\tau_{A B}\right)$ points away from particle $A, s_{v}=+1$, the mediatorino can hit particle $T$ only when $\mathbf{x}_{m}\left(\tau_{A}\right)$ is in $\mathscr{C}_{w, c}^{+}$or in $\mathscr{S}_{w}$. On the other hand, if velocity $\mathbf{v}_{m}\left(\tau_{A B}\right)$ points toward particle $A, s_{v}=-1$, the mediatorino can hit particle $T$ only when $\mathbf{x}_{m}\left(\tau_{A}\right)$ is in $\mathscr{C}_{w}^{-}, \mathscr{S}_{w}$, or $\mathscr{C}_{w, f}^{+}$. In total,

$$
\begin{aligned}
& K_{i, B}^{++}\left(\mathbf{r}_{T}, \hat{\mathbf{e}}\right)= \begin{cases}K_{i, A}^{+}\left(\mathbf{r}_{S},-\hat{\mathbf{e}}\right), & \text { if } \mathbf{x}_{m}\left(\tau_{A}\right) \in \mathscr{C}_{w, c}^{+} \cup \mathscr{S}_{w} \wedge \hat{\mathbf{e}} \in E_{w}^{+}, \\
0, & \text { otherwise, }\end{cases} \\
& K_{i, B}^{+-}\left(\mathbf{r}_{T}, \hat{\mathbf{e}}\right)= \begin{cases}K_{i, A}^{+}\left(\mathbf{r}_{S},-\hat{\mathbf{e}}\right), & \text { if } \mathbf{x}_{m}\left(\tau_{A}\right) \in \mathscr{S}_{w} \cup \mathscr{C}_{w, f}^{+} \wedge \hat{\mathbf{e}} \in E_{w}^{+}, \\
K_{i, A}^{+}\left(\mathbf{r}_{S},-\hat{\mathbf{e}}\right), & \text { if } \mathbf{x}_{m}\left(\tau_{A}\right) \in \mathscr{C}_{w}^{-} \wedge \hat{\mathbf{e}} \in E_{w}^{-}, \\
0, & \text { otherwise. }\end{cases}
\end{aligned}
$$

The concrete form of $K_{i, B}^{++}$and $K_{i, B}^{+-}$depends on the specific transfer. In transfers $I_{18}$ and $I_{26}$ mediatorino $m$ has at time $\tau_{A}$ a single location (on particle $B$ ) and a single direction $\hat{\mathbf{v}}_{m}$. In transfers $I_{2}$ and $I_{10}$ the mediatorino has at time $\tau_{A}$ a single location (on particle $A$ ) but 
arbitrary direction $\hat{\mathbf{v}}_{m}$, and finally in transfers $I_{6}, I_{14}, I_{22}$, and $I_{30}$ mediatorino $m$ can at time $\tau_{A}$ be at various locations (on the surface of sphere $S_{R}$ ) and to each of them corresponds a single direction $\hat{\mathbf{v}}_{m}$. The further analysis of hitting particle $T$ is, therefore, saved for the analysis of the individual transfers.

\subsection{Frequencies}

The mean frequency $\left\langle v_{i, 0}\right\rangle$ of the initiation of information transfer $I_{i}$ can be different from the mean frequency $\left\langle v_{i, A}\right\rangle$ of taking $\triangle \mathbf{q}$ from particle $A$. This happens in the transfers with $\tau_{0}<\tau_{A}=\tau_{E}$ where mediatorino $m$ must hit particle $T$ in order to take $\Delta \mathbf{q}$ from particle $A$. The frequency $\left\langle v_{i, A}\right\rangle$ is, therefore, influenced by Doppler effect caused by the speed of particle $T$. The Doppler effect for the transfers with $\tau_{0}<\tau_{A}=\tau_{E}$ can be expressed as [34]

$$
\left\langle v_{i, A}\right\rangle=\left\langle v_{i, 0}\right\rangle \cdot\left(\frac{v_{m}\left(\tau_{0 A}\right)-\mathbf{v}_{T}\left(\tau_{A}\right) \cdot \mathbf{n}_{0 A}}{v_{m}\left(\tau_{0 A}\right)-\mathbf{v}_{S}\left(\tau_{0}\right) \cdot \mathbf{n}_{0 A}}\right),
$$

where vector $\mathbf{n}_{0 A}$ is

$$
\mathbf{n}_{0 A}=\mathbf{x}_{T}\left(\tau_{A}\right)-\mathbf{x}_{m}\left(\tau_{0}\right) .
$$

In the information transfers with $\tau_{0}=\tau_{A}, \triangle \mathbf{q}$ is taken from particle $A$ at the beginning of the transfer. Similarly, in the transfers with $\tau_{0}<\tau_{A}<\tau_{E}$ the value of $\triangle \mathbf{q}$ is taken from particle $A$ after a constant time $\tau_{A}-\tau_{0}$. In both cases, the frequency of taking $\triangle \mathbf{q}$ from particle $A$ is the same as the frequency of the initiation of the transfer. That is, for the transfers with $\tau_{0}=\tau_{A}$ or with $\tau_{0}<\tau_{A}<\tau_{E}$,

$$
\left\langle v_{i, A}\right\rangle=\left\langle v_{i, 0}\right\rangle .
$$

The mean frequency $\left\langle v_{i, A}\right\rangle$ of taking $\triangle \mathbf{q}$ from particle $A$ can be different from the mean frequency $\left\langle v_{i, B}\right\rangle$ of putting $\triangle \mathbf{q}$ onto particle $B$. For the transfers with $\tau_{A}<\tau_{B}=\tau_{E}$ where mediatorino $m$ must hit particle $T$ in order to put $\triangle \mathbf{q}$ into particle $B$, Doppler effect must be incorporated:

$$
\left\langle v_{i, B}\right\rangle=\left\langle v_{i, A}\right\rangle \cdot\left(\frac{v_{m}\left(\tau_{A B}\right)-\mathbf{v}_{T}\left(\tau_{B}\right) \cdot \mathbf{n}_{A B}}{v_{m}\left(\tau_{A B}\right)-\mathbf{v}_{S}\left(\tau_{0}\right) \cdot \mathbf{n}_{A B}}\right),
$$

where $\mathbf{n}_{A B}$ satisfies

$$
\mathbf{n}_{A B}=\mathbf{x}_{T}\left(\tau_{B}\right)-\mathbf{x}_{m}\left(\tau_{A}\right) .
$$

In the other transfers, i.e., in the transfers with $\tau_{B}<\tau_{E}$ and in the transfers with $\tau_{0}<\tau_{A}=\tau_{E}, \Delta \mathbf{q}$ is transferred to particle $B$ via an entanglement. In the case of $\tau_{A}=\tau_{B}<\tau_{E}$ or the case of $\tau_{0}<\tau_{A}=\tau_{E}, \Delta \mathbf{q}$ is transferred to particle $B$ immediately when it is taken from particle $A$, and in the case of $\tau_{A}<\tau_{B}<\tau_{E}$, it is transferred after a constant time $\tau_{B}-\tau_{A}$. In both cases,

$$
\left\langle v_{i, B}\right\rangle=\left\langle v_{i, A}\right\rangle .
$$

The differences of $\left\langle v_{i, 0}\right\rangle,\left\langle v_{i, A}\right\rangle$, and $\left\langle v_{i, B}\right\rangle$ influence the differences of $f_{i, 0}, f_{i, A}$ and $f_{i, B}$ via equation (41).

\subsection{Force on particle $B$ in transfers $I_{2}$ and $I_{10}$}

Information transfers $I_{2}$ and $I_{10}$ satisfy $S=A$ and $\tau_{0}=\tau_{A}<\tau_{B}=\tau_{E}$. Vector $\mathbf{z}$ is defined by equation (56). Let $\mathbf{r}_{T}=\mathbf{z}$. For $\mathbf{x}_{m}\left(\tau_{A}\right)=\mathbf{x}_{A}\left(\tau_{A}\right)$ mediatorino $m$ at time $\tau_{A}$ starts to move 
always from particle $A$, i.e., $s_{v}=-1$ has no good meaning. Therefore, we use only $s_{v}=+1$. For $r_{T} \leq w, K_{i, B}^{++}$is given by equation (104). Since $\mathbf{x}_{m}\left(\tau_{A}\right)=\mathbf{x}_{A}\left(\tau_{A}\right)$, point $\mathbf{x}_{m}\left(\tau_{A}\right)$ is automatically in sphere $\mathscr{S}_{w}$. We get

$$
K_{i, B}^{++}\left(\mathbf{r}_{T}, \hat{\mathbf{e}}\right)=K_{i, A}^{+}\left(\mathbf{r}_{S},-\hat{\mathbf{e}}\right) .
$$

For $r_{T}>w$ distribution $K_{i, B}^{++}$is given by equation (109). Since $\mathbf{x}_{m}\left(\tau_{A}\right)=\mathbf{x}_{A}\left(\tau_{A}\right)$, there is $\mathbf{x}_{m}\left(\tau_{A}\right) \in \mathscr{C}_{w, c}^{+}$. It leads to

$$
K_{i, B}^{++}\left(\mathbf{r}_{T}, \hat{\mathbf{e}}\right)= \begin{cases}K_{i, A}^{+}\left(\mathbf{r}_{S},-\hat{\mathbf{e}}\right), & \text { if } \hat{\mathbf{e}} \in E_{w}^{+}, \\ 0, & \text { otherwise. }\end{cases}
$$

In transfers $I_{2}$ and $I_{10}, K_{i, A}^{+}$is given by equation (57). Therefore, the directional distribution of a change of $\mathbf{q}$ on particle $B$ for $i \in\{2,10\}$ can finally be written as

$$
K_{i, B}^{++}\left(\mathbf{r}_{T}, \hat{\mathbf{e}}\right)= \begin{cases}1 / 4 \pi, & \text { if } r_{T} \leq w, \\ 1 / 4 \pi, & \text { if } r_{T}>w, \hat{\mathbf{e}} \in E_{w}^{+}, \\ 0, & \text { otherwise. }\end{cases}
$$

It can be used to calculate $\Gamma_{i, B}^{j++}$ and $\Gamma_{i, B}^{k l++}$. While for $r_{T} \leq w$ we have

$$
\begin{aligned}
\Gamma_{i, B}^{j++} & =(0,0,0)^{T}, \\
\Gamma_{i, B}^{k l++} & =\left(\begin{array}{ccc}
1 / 3 & 0 & 0 \\
0 & 1 / 3 & 0 \\
0 & 0 & 1 / 3
\end{array}\right),
\end{aligned}
$$

for $r_{T}>w$ we have

$$
\begin{aligned}
\Gamma_{i, B}^{3++} & =\frac{w^{2}}{4 r_{T}^{2}}, \\
\Gamma_{i, B}^{11++}=\Gamma_{i, B}^{22++} & =\frac{1}{6}\left[1-\sqrt{1-\frac{w^{2}}{r_{T}^{2}}}\left(1+\frac{w^{2}}{2 r_{T}^{2}}\right)\right], \\
\Gamma_{i, B}^{33++} & =\frac{1}{6}\left[1-\left(1-\frac{w^{2}}{r_{T}^{2}}\right)^{3 / 2}\right] .
\end{aligned}
$$

Moreover, for $r_{T} \gg w, \Gamma_{i, B}^{11++}, \Gamma_{i, B}^{22++}$ and $\Gamma_{i, B}^{33++}$ can be expressed in terms of order no higher than $w^{4} / r_{T}^{4}$ as

$$
\begin{aligned}
\Gamma_{i, B}^{11++}=\Gamma_{i, B}^{22++} & \sim \frac{w^{4}}{16 r_{T}^{4}}, \\
\Gamma_{i, B}^{33++} & \sim \frac{w^{2}}{4 r_{T}^{2}}-\frac{w^{4}}{16 r_{T}^{4}} .
\end{aligned}
$$

When we calculate the mean force for $i \in\{2,10\}$, we get

$$
\left\langle\mathbf{F}_{i, B}^{++}\left(\mathbf{r}_{T}\right)\right\rangle= \begin{cases}\mathbf{0}, & \text { if } 0 \leq r_{T} \leq w, \\ \frac{f_{i, B} w^{2}}{4 r_{T}^{2}} \cdot \hat{\mathbf{z}}, & \text { if } r_{T}>w .\end{cases}
$$

We see that although transfers $I_{2}$ and $I_{10}$ behave at large distances proportionally to the inverse square of distance $r_{T}$, there is no divergence for short distances $r_{T}$. 
7.19 Force on particle $B$ in transfers $I_{18}$ and $I_{26}$

Information transfers $I_{18}$ and $I_{26}$ satisfy $S=B$ and $\tau_{0}=\tau_{A}<\tau_{B}=\tau_{E}$. Vector $\mathbf{z}$ is defined by equation (61) and vector $\mathbf{r}_{T}$ is defined by

$$
\mathbf{r}_{T}=\mathbf{x}_{A}\left(\tau_{B}\right)-\mathbf{x}_{A}\left(\tau_{A}\right),
$$

which can be written in terms of the average velocity $\left\langle\mathbf{v}_{A}\left(\tau_{A B}\right)\right\rangle$ as

$$
\mathbf{r}_{T}=\left\langle\mathbf{v}_{A}\left(\tau_{A B}\right)\right\rangle \cdot\left(\tau_{B}-\tau_{A}\right) .
$$

Mediatorino $m$ is at time $\tau_{A}$ on particle $B$ and during time interval $\left(\tau_{A}, \tau_{B}\right)$ travels along line $\mathbf{I}$ defined by equation (24). In order to transfer $\triangle \mathbf{q}$ to particle $B$ the mediatorino needs to hit particle $T=A$. Since due to equation (64), $K_{i, A}^{+}$is non-zero only for a single direction, and since mediatorino $m$ is at time $\tau_{A}$ on a point-like particle, we can use equation (101) to write

$$
K_{i, B}^{+ \pm}=P_{i, B}^{+ \pm} \cdot \delta_{d}(\vartheta, \varphi)
$$

where $P_{i, B}^{+ \pm}$is the probability that mediatorino $m$ hits particle $A$. The corresponding $\Gamma_{i, B}^{j+ \pm}$, $\Gamma_{i, B}^{k l+ \pm}$ and $\left\langle\mathbf{F}_{i, B}^{+ \pm}\right\rangle$are

$$
\begin{aligned}
& \Gamma_{i, B}^{j+ \pm}=P_{i, B}^{+ \pm} \cdot(0,0,1)^{T}, \\
& \Gamma_{i, B}^{k l+ \pm}=P_{i, B}^{+ \pm} \cdot\left(\begin{array}{lll}
0 & 0 & 0 \\
0 & 0 & 0 \\
0 & 0 & 1
\end{array}\right), \\
& \left\langle\mathbf{F}_{i, B}^{+ \pm}\right\rangle=f_{i, B} \cdot P_{i, B}^{+ \pm} \cdot \hat{\mathbf{z}} .
\end{aligned}
$$

Now we only need to express $P_{i, B}^{+ \pm}$.

For $r_{T} \leq w$ particle $A$ is practically static and stays on or very close to line $\mathbf{l}$. If $\mathbf{v}_{m}\left(\tau_{A B}\right)$ points away from particle $A, s_{v}=+1$, then the mediatorino is getting during time interval $\left(\tau_{A}, \tau_{B}\right)$ farther from particle $A$. Consequently, mediatorino $m$ never hits particle $T$. On the other hand, if $\mathbf{v}_{m}\left(\tau_{A B}\right)$ points toward particle $A, s_{v}=-1$, then the mediatorino is getting during $\left(\tau_{A}, \tau_{B}\right)$ closer to particle $A$, and eventually hits particle $T$. In total, for $i \in\{18,26\}$ :

$$
\begin{aligned}
& P_{i, B}^{++}=0, \\
& P_{i, B}^{+-}=1 .
\end{aligned}
$$

This exhibits an asymmetry with respect to a change of the sign of $s_{v}$.

For $r_{T}>w$ equations (109) and (110) describe the situation completely, nevertheless they do not provide any quick insight into what is going on in this case. Moreover, due to equation (129), the result highly depends on the behavior of particle $A$. Therefore, we choose to investigate a specific system rather than a general case. Namely, we investigate a system where the direction of the velocity of particle $A$ rapidly changes and the distribution of the directions of the velocities $\mathbf{v}_{A}\left(\tau_{A B}\right)$ is uniform, such as in the case when particle $A$ is within a small spherical symmetric bound system. The mediatorino hits particle $A$ only when the mediatorino is on the surface or inside double cone $\mathscr{C}_{w}\left(\mathbf{x}_{A}\left(\tau_{A}\right), \mathbf{r}_{T}, w\right)$. For mediatorino $m$, the probability of being within a single arm of the double cone $\mathscr{C}_{w}$ with randomly oriented axis and solid angle $\Omega_{w}$ is $P_{w}=\Omega_{w} / 4 \pi$. Since $\Omega_{w}$ is given by equation (108), we have

$$
P_{w}=\frac{1}{2}\left(1-\sqrt{1-\frac{w^{2}}{r_{T}^{2}}}\right) \text {. }
$$


In addition to being in an arm of the double cone, there is also important the fact in what region $\left(\mathscr{C}_{w}^{-}, \mathscr{C}_{w, c}^{+}, \mathscr{S}_{w}, \mathscr{C}_{w, f}^{+}\right)$mediatorino $m$ is located at time $\tau_{A}$. It depends on distance $l$ of the mediatorino from $\mathbf{x}_{A}\left(\tau_{A}\right)$, the apex of the double cone $\mathscr{C}_{w}$. For most distances $l$ the probability $P_{i, B}^{+ \pm}$can be calculated from $P_{w}$, nevertheless there are two exceptions. Namely, in the case of $P_{i, B}^{+-}$for $l \in\left(r_{T}-w, \sqrt{r_{T}^{2}-w^{2}}\right)$ and in the case of $P_{i, B}^{++}$for $l \in\left(\sqrt{r_{T}^{2}-w^{2}}, r_{T}+w\right)$. In these cases, the probability is proportional to the intersection of a sphere with center at $\mathbf{x}_{A}\left(\tau_{A}\right)$ and radius $l$ and sphere $\mathscr{S}_{w}$ with radius $w$. The surface of a sphere with radius $l$ which is intersected by a sphere with radius $w$ is

$$
S_{l w}=2 \pi l\left(l-\frac{r_{T}^{2}+l^{2}-w^{2}}{2 r_{T}}\right),
$$

provided $r_{T}$ is the distance of the sphere centers. Since for a mediatorino being on the surface of the sphere with radius $l$ the probability of being on the intersecting surface $S_{l w}$ is $P_{l w}=S_{l w} / 4 \pi l^{2}$, we obtain

$$
P_{l w}=\frac{1}{2}\left(1-\frac{r_{T}^{2}+l^{2}-w^{2}}{2 r_{T} l}\right) .
$$

When we decompose equations (109) and (110) into their regions, convert the regions into the corresponding distances $l$ and merge, we get

$$
\begin{aligned}
& P_{i, B}^{++}= \begin{cases}P_{w}, & l \in\left(0, \sqrt{r_{T}^{2}-w^{2}}\right], \\
P_{l w}, & l \in\left[\sqrt{r_{T}^{2}-w^{2}}, r_{T}+w\right], \\
0, & l \in\left[r_{T}+w, \infty\right),\end{cases} \\
& P_{i, B}^{+-}= \begin{cases}P_{w}, & l \in\left(0, r_{T}-w\right], \\
P_{w}+P_{l w}, & l \in\left[r_{T}-w, \sqrt{r_{T}^{2}-w^{2}}\right], \\
2 P_{w}, & l \in\left[\sqrt{r_{T}^{2}-w^{2}}, \infty\right) .\end{cases}
\end{aligned}
$$

We again see an asymmetry with respect to a change of the sign of $s_{v}$, this time for $l>r_{T}-w$. For $r_{T} \gg w$ we can ignore the tiny transitional middle interval $l \in\left[r_{T}-w, r_{T}+w\right]$ and in terms of $w / r_{T}$ write

$$
\begin{aligned}
& P_{i, B}^{++} \sim\left\{\begin{array}{l}
\frac{w^{2}}{4 r_{T}^{2}}, l \in\left(0, r_{T}\right), \\
0, \quad l \in\left(r_{T}, \infty\right),
\end{array}\right. \\
& P_{i, B}^{+-} \sim\left\{\begin{array}{l}
\frac{w^{2}}{4 r_{T}^{2}}, l \in\left(0, r_{T}\right), \\
\frac{w^{2}}{2 r_{T}^{2}}, l \in\left(r_{T}, \infty\right) .
\end{array}\right.
\end{aligned}
$$

This, along with equations (131) to (133), gives us the wanted quick overview of the case.

7.20 Force on particle $B$ in $I_{6}, I_{14}, I_{22}$ and $I_{30}$

Information transfers $I_{6}, I_{14}, I_{22}$ and $I_{30}$ satisfy $\tau_{0}<\tau_{A}<\tau_{B}=\tau_{E}$. Vectors $\mathbf{z}$ and $\mathbf{r}_{S}$ are defined by equation (69) and vector $\mathbf{r}_{T}$ is defined by equation (102). Since the transfers 
satisfy $\tau_{0}<\tau_{A}<\tau_{E}$, the movement of their mediatorino $m$ during $\left(\tau_{A}, \tau_{B}\right)$ is bounded by $\mathscr{C}_{R}=\mathscr{C}\left(\mathbf{x}_{A}\left(\tau_{A}\right), \mathbf{r}_{S}, R\right)$. The transfers also satisfy $\tau_{A}<\tau_{B}=\tau_{E}$, and thus $\Delta \mathbf{q}$ is transferred to particle $B$ only when mediatorino $m$ hits target particle $T$, that is when mediatorino $m$ moves within $\mathscr{C}_{w}=\mathscr{C}\left(\mathbf{x}_{A}\left(\tau_{A}\right), \mathbf{r}_{T}, w\right)$. In total, $\triangle \mathbf{q}$ is transferred to particle $B$ when mediatorino $m$ moves within $\mathscr{C}_{R} \cap \mathscr{C}_{w}$. Different locations of $\mathbf{x}_{A}\left(\tau_{A}\right), \mathbf{x}_{T}\left(\tau_{E}\right)$ and $\mathbf{x}_{S}\left(\tau_{0}\right)$, different sizes of $w$ and $R$, and different values of $s_{v}$ give rise to several tens of possible situations. Information transfers $I_{6}, I_{14}, I_{22}$ and $I_{30}$ are, therefore, by far the most complex of all the studied information transfers. We do not study all the situations, we focus only on few of them. Namely, we examine the situations where locality parameter $w$ is substantially smaller than radius $R, w \ll R$, and ignore all the situations where sphere $\mathscr{S}_{w}$ intersects any region of $\mathscr{C}_{R}$ partially (regardless of whether $\mathscr{C}_{R}$ is a double cone or full space). Moreover, in the case of $r_{T}>w$, we examine only $r_{T} \gg w$. There still remain 18 situations to be examined.

For $r_{T} \leq w$ point $\mathbf{x}_{A}\left(\tau_{A}\right)$ is inside sphere $\mathscr{S}_{w}$. There are four possible situations (without partial intersection of spheres $\mathscr{S}_{R}$ and $\left.\mathscr{S}_{w}\right)$ which come from two regions where $\mathbf{x}_{T}\left(\tau_{E}\right)$ can be located $\left(\mathscr{S}_{R}\right.$ and $\left.\mathbb{R}^{3} \backslash \mathscr{S}_{R}\right)$ and two variants of $s_{v}(+1$ and -1$)$. The cases are as follows: (1) $\mathbf{x}_{T}\left(\tau_{E}\right) \in \mathscr{S}_{R}, s_{v}=+1$ : Mediatorinos $m$ go from the surface of $\mathscr{S}_{R}$ away from particle $A$. Since due to $r_{T} \leq w$ particle $A$ is inside sphere $\mathscr{S}_{w}$, the mediatorinos go away from sphere $\mathscr{S}_{w}$ and will never hit particle $T$. (2) $\mathbf{x}_{T}\left(\tau_{E}\right) \in \mathscr{S}_{R}, s_{v}=-1$ : Mediatorinos $m$ go from the surface of $\mathscr{S}_{R}$ toward particle $A$. Since $r_{T} \leq w$, the mediatorinos go toward sphere $\mathscr{S}_{w}$ and will eventually hit particle $T$. (3) $\mathbf{x}_{T}\left(\tau_{E}\right) \notin \mathscr{S}_{R}, s_{v}=+1$ : Similar to the first case, mediatorinos $m$ go from the surface of $\mathscr{S}_{R}$ away from sphere $\mathscr{S}_{w}$, and thus they will never hit particle $T$. (4) $\mathbf{x}_{T}\left(\tau_{E}\right) \notin \mathscr{S}_{R}, s_{v}=-1$ : Similar to the second case, mediatorinos $m$ go from the surface of $\mathscr{S}_{R}$ away from sphere $\mathscr{S}_{w}$, and will eventually hit particle $T$. In total, for $r_{T} \leq w$ we get

$$
\begin{aligned}
& K_{i, B}^{++}\left(\mathbf{r}_{T}, \hat{\mathbf{e}}\right)=0, \\
& K_{i, B}^{+-}\left(\mathbf{r}_{T}, \hat{\mathbf{e}}\right)=K_{i, A}^{+}\left(\mathbf{r}_{S},-\hat{\mathbf{e}}\right) .
\end{aligned}
$$

The corresponding $\Gamma_{i, B}^{j+ \pm}$ is

$$
\begin{aligned}
& \Gamma_{i, B}^{j++}=(0,0,0)^{T}, \\
& \Gamma_{i, B}^{j+-}=\Gamma_{s p, A}^{j+},
\end{aligned}
$$

where $\Gamma_{s p, A}^{j+}$ is given by equation (79), and the corresponding $\Gamma_{i, B}^{k l+ \pm}$ is

$$
\begin{aligned}
& \Gamma_{i, B}^{k l++}=\left(\begin{array}{lll}
0 & 0 & 0 \\
0 & 0 & 0 \\
0 & 0 & 0
\end{array}\right), \\
& \Gamma_{i, B}^{k l+-}=\Gamma_{s p, A}^{k l+},
\end{aligned}
$$

where $\Gamma_{s p, A}^{k l+}$ is given by equations (47), (80) and (81). Finally, the corresponding average force is

$$
\begin{aligned}
\left\langle\mathbf{F}_{i, B}^{++}\left(\mathbf{r}_{T}\right)\right\rangle & =\mathbf{0}, \\
\left\langle\mathbf{F}_{i, B}^{+-}\left(\mathbf{r}_{T}\right)\right\rangle & =-\left\langle\mathbf{F}_{i, A}^{+}\left(\mathbf{r}_{S}\right)\right\rangle .
\end{aligned}
$$

For $r_{T}>w$ point $\mathbf{x}_{A}\left(\tau_{A}\right)$ is outside sphere $\mathscr{S}_{w}$. We examine only $r_{T} \gg w$, that is double cone $\mathscr{C}_{w}$ has a very small solid angle $\Omega_{w}$. This case contains 14 situations, 4 situations for 
$r_{S} \leq R$ and 10 situations for $r_{S}>R$. Let us define $\mathbf{x}_{m}(\hat{\mathbf{r}})$ as the location of mediatorino $m$ on the surface of sphere $\mathscr{S}_{R}$ which is in direction $\hat{\mathbf{r}}$ from $\mathbf{x}_{A}\left(\tau_{A}\right)$. For $r_{T}>w$ and $r_{S} \leq R$ point $\mathbf{x}_{A}\left(\tau_{A}\right)$ is inside sphere $\mathscr{S}_{R}$. In that case, we distinguish four situations which come from two regions where $\mathbf{x}_{T}\left(\tau_{E}\right)$ can be located $\left(\mathscr{S}_{R}\right.$ and $\left.\mathbb{R}^{3} \backslash \mathscr{S}_{R}\right)$ and two variants of $s_{v}$ (+1 and -1). The individual cases are the following: (5) $\mathbf{x}_{T}\left(\tau_{E}\right) \in \mathscr{S}_{R}, s_{v}=+1$ : Sphere $S_{w}$ is (due to $\mathbf{x}_{T} \in \mathscr{S}_{R}$ ) inside sphere $\mathscr{S}_{R}$; however, all mediatorinos $m$ are at time $\tau_{A}$ on the surface of $\mathscr{S}_{R}$ and (due to $s_{v}=+1$ ) go away from particle $A$. Since particle $A$ is (due to $r_{S}<R$ ) inside sphere $\mathscr{S}_{R}$, no mediatorino can ever hit particle $T$. (6) $\mathbf{x}_{T}\left(\tau_{E}\right) \in \mathscr{S}_{R}$, $s_{v}=-1$ : Sphere $S_{w}$ is inside sphere $\mathscr{S}_{R}$ and all mediatorinos $m$ are at time $\tau_{A}$ on the surface of $\mathscr{S}_{R}$ and go toward particle $A$. Since particle $A$ is inside sphere $\mathscr{S}_{R}$, mediatorinos located close to $\mathbf{x}_{m}\left(\hat{\mathbf{r}}_{T}\right)$ and $\mathbf{x}_{m}\left(-\hat{\mathbf{r}}_{T}\right)$ can hit particle $T$. To be close to point $\mathbf{x}_{m}$ here means that they are within the intersection of the corresponding arm of double cone $\mathscr{C}_{w}$ and the surface of sphere $\mathscr{S}_{R}$. (7) $\mathbf{x}_{T}\left(\tau_{E}\right) \notin \mathscr{S}_{R}, s_{v}=+1$ : Sphere $S_{w}$ is outside sphere $\mathscr{S}_{R}$ and all mediatorinos $m$ are at time $\tau_{A}$ on the surface of $\mathscr{S}_{R}$ and go away from particle $A$. Since particle $A$ is inside sphere $\mathscr{S}_{R}$, only mediatorinos located close to $\mathbf{x}_{m}\left(\hat{\mathbf{r}}_{T}\right)$ can hit particle $T$. (8) $\mathbf{x}_{T}\left(\tau_{E}\right) \notin \mathscr{S}_{R}$, $s_{v}=-1$ : Sphere $S_{w}$ is outside sphere $\mathscr{S}_{R}$ and mediatorinos $m$ are at time $\tau_{A}$ on the surface of $\mathscr{S}_{R}$ and go toward particle $A$. Since particle $A$ is inside sphere $\mathscr{S}_{R}$, mediatorino $m$ located close to $\mathbf{x}_{m}\left(-\hat{\mathbf{r}}_{T}\right)$ can hit particle $T$. For $r_{T}>w$ and $r_{S}>R, \mathscr{C}_{R}$ is a double cone. Now we distinguish ten situations which come from five regions where $\mathbf{x}_{T}\left(\tau_{E}\right)$ can be located $\left(\mathscr{C}_{R}^{-}\right.$, $\mathscr{C}_{R, c}^{+}, \mathscr{S}_{R}, \mathscr{C}_{R, f}^{+}$and $\left.\mathbb{R}^{3} \backslash \mathscr{C}_{R}\right)$ and two variants of $s_{v}(+1$ and -1$)$. Let us define $\mathbf{x}_{m-}(\hat{\mathbf{r}})$ and $\mathbf{x}_{m+}(\hat{\mathbf{r}})$ as the locations of mediatorino $m$ on the surface of sphere $\mathscr{S}_{R}$ which are from point $\mathbf{x}_{A}\left(\tau_{A}\right)$ in direction $\hat{\mathbf{r}}$ and at distance $l_{-}$and $l_{+}$, respectively, provided $l_{-}$and $l_{+}$are given by equation (75). The individual situations are: (9) $\mathbf{x}_{T}\left(\tau_{E}\right) \in \mathscr{C}_{R, f}^{+}, s_{v}=+1$ : Mediatorinos $m$ go from the surface of $\mathscr{S}_{R}$ away from particle $A$. Since sphere $\mathscr{S}_{w}$ is in $\mathscr{C}_{R, f}^{+}$which is farther from point $\mathbf{x}_{A}\left(\tau_{A}\right)$ than sphere $\mathscr{S}_{R}$, then mediatorinos $m$ can hit particle $T$ when the mediatorinos are at time $\tau_{A}$ located close to $\mathbf{x}_{m-}\left(\hat{\mathbf{r}}_{T}\right)$ or $\mathbf{x}_{m+}\left(\hat{\mathbf{r}}_{T}\right)$. (10) $\mathbf{x}_{T}\left(\tau_{E}\right) \in \mathscr{C}_{R, f}^{+}$, $s_{v}=-1$ : Mediatorinos $m$ go from the surface of $\mathscr{S}_{R}$ toward particle $A$. Since sphere $\mathscr{S}_{w}$ is in $\mathscr{C}_{R, f}^{+}$which is farther from point $\mathbf{x}_{A}\left(\tau_{A}\right)$ than sphere $\mathscr{S}_{R}$, then no mediatorino can hit particle $T$. (11) $\mathbf{x}_{T}\left(\tau_{E}\right) \in \mathscr{S}_{R}, s_{v}=+1$ : Mediatorinos $m$ go from the surface of $\mathscr{S}_{R}$ away from particle $A$. Since sphere $\mathscr{S}_{w}$ is inside sphere $\mathscr{S}_{R}$, only the mediatorinos that are at time $\tau_{A}$ located close to $\mathbf{x}_{m-}\left(\hat{\mathbf{r}}_{T}\right)$ can hit particle $T$. (12) $\mathbf{x}_{T}\left(\tau_{E}\right) \in \mathscr{S}_{R}, s_{v}=-1$ : Mediatorinos $m$ go from the surface of $\mathscr{S}_{R}$ toward particle $A$. Since sphere $\mathscr{S}_{w}$ is inside sphere $\mathscr{S}_{R}$, only the mediatorinos that are at time $\tau_{A}$ located close to $\mathbf{x}_{m+}\left(\hat{\mathbf{r}}_{T}\right)$ can hit particle $T$. (13) $\mathbf{x}_{T}\left(\tau_{E}\right) \in \mathscr{C}_{R, c}^{+}, s_{v}=+1$ : Mediatorinos $m$ go from the surface of $\mathscr{S}_{R}$ away from particle $A$. Since sphere $\mathscr{S}_{w}$ is in $\mathscr{C}_{R, c}^{+}$which is closer to point $\mathbf{x}_{A}\left(\tau_{A}\right)$ than sphere $\mathscr{S}_{R}$, then no mediatorino can hit particle $T$. (14) $\mathbf{x}_{T}\left(\tau_{E}\right) \in \mathscr{C}_{R, c}^{+}, s_{v}=-1$ : Mediatorinos $m$ go from the surface of $\mathscr{S}_{R}$ toward particle $A$. Since sphere $\mathscr{S}_{w}$ is in $\mathscr{C}_{R, c}^{+}$which is closer to point $\mathbf{x}_{A}\left(\tau_{A}\right)$ than sphere $\mathscr{S}_{R}$, the mediatorinos that are at time $\tau_{A}$ close to $\mathbf{x}_{m-}\left(\hat{\mathbf{r}}_{T}\right)$ and $\mathbf{x}_{m+}\left(\hat{\mathbf{r}}_{T}\right)$ can hit particle $T$. (15) $\mathbf{x}_{T}\left(\tau_{E}\right) \in \mathscr{C}_{R}^{-}, s_{v}=+1$ : Mediatorinos $m$ go from the surface of $\mathscr{S}_{R}$ away from particle $A$. Since $\mathscr{C}_{R}^{-}$is from $\mathbf{x}_{A}\left(\tau_{A}\right)$ on the opposite side than $\mathscr{S}_{R}$, no mediatorino can hit particle $T$. (16) $\mathbf{x}_{T}\left(\tau_{E}\right) \in \mathscr{C}_{R}^{-}, s_{v}=-1$ : Mediatorinos $m$ go from the surface of $\mathscr{S}_{R}$ toward particle $A$. Even $\mathscr{C}_{R}^{-}$is from $\mathbf{x}_{A}\left(\tau_{A}\right)$ on the opposite side than $\mathscr{S}_{R}$, the mediatorinos that are close to $\mathbf{x}_{m-}\left(\hat{\mathbf{r}}_{T}\right)$ and $\mathbf{x}_{m+}\left(\hat{\mathbf{r}}_{T}\right)$ can hit particle $T$. (17) $\mathbf{x}_{T}\left(\tau_{E}\right) \in \mathbb{R}^{3} \backslash \mathscr{C}_{R}, s_{v}=-1$ : Since the intersection of double cones $\mathscr{C}_{w}$ and $\mathscr{C}_{R}$ is empty, mediatorinos $m$ cannot hit particle $T$. (18) $\mathbf{x}_{T}\left(\tau_{E}\right) \in \mathbb{R}^{3} \backslash \mathscr{C}_{R}, s_{v}=-1$ : Similar to the previous case, since the intersection of 
$\mathscr{C}_{w}$ and $\mathscr{C}_{R}$ is empty, no mediatorino can hit particle $T$. In total, for $r_{T} \gg w$ we get

$$
\begin{aligned}
K_{i, B}^{++}\left(\mathbf{r}_{T}, \hat{\mathbf{e}}\right)= \begin{cases}\left|k_{+}\left(r_{S}, \vartheta\right)\right|, & \text { if } r_{S}<R, \hat{\mathbf{e}} \in E_{w}^{+}, \mathbf{x}_{T} \in \mathbb{R}^{3}-\mathscr{S}_{R}, \\
\left|k_{-}\left(r_{S}, \vartheta\right)\right|, & \text { if } r_{S}>R, \hat{\mathbf{e}} \in E_{w}^{+}, \mathbf{x}_{T} \in \mathscr{S}_{R}, \\
\left|k_{+}\left(r_{S}, \vartheta\right)\right|+\left|k_{-}\left(r_{S}, \vartheta\right)\right|, & \text { if } r_{S}>R, \hat{\mathbf{e}} \in E_{w}^{+}, \mathbf{x}_{T} \in \mathscr{C}_{R, f}^{+}, \\
0, & \text { otherwise, }\end{cases} \\
K_{i, B}^{+-}\left(\mathbf{r}_{T}, \hat{\mathbf{e}}\right)= \begin{cases}\left|k_{+}\left(r_{S}, \vartheta\right)\right|, & \text { if } r_{S}<R, \hat{\mathbf{e}} \in E_{w}^{+} \cup E_{w}^{-}, \mathbf{x}_{T} \in \mathscr{S}_{R}, \\
\left|k_{+}\left(r_{S}, \vartheta\right)\right|, & \text { if } r_{S}<R, \hat{\mathbf{e}} \in E_{w}^{-}, \mathbf{x}_{T} \in \mathbb{R}^{3}-\mathscr{S}_{R}, \\
\left|k_{+}\left(r_{S}, \vartheta\right)\right|, & \text { if } r_{S}>R, \hat{\mathbf{e}} \in E_{w}^{+}, \mathbf{x}_{T} \in \mathscr{S}_{R}, \\
\left|k_{+}\left(r_{S}, \vartheta\right)\right|+\left|k_{-}\left(r_{S}, \vartheta\right)\right|, & \text { if } r_{S}>R, \hat{\mathbf{e}} \in E_{w}^{+}, \mathbf{x}_{T} \in \mathscr{C}_{R}^{-} \cup \mathscr{C}_{R, c}^{+}, \\
0, & \text { otherwise, }\end{cases}
\end{aligned}
$$

where $R$ is given by equation (68), $k_{+}$and $k_{-}$are given by equation (77), $\hat{\mathbf{e}}=\hat{\mathbf{e}}(\vartheta, \varphi)$ is defined by equation (31), $\mathbf{x}_{T}=\mathbf{x}_{T}\left(\tau_{E}\right)$, and $E_{w}^{ \pm}=E\left(\mathbf{x}_{A}\left(\tau_{A}\right), \pm \mathbf{r}_{T}, w\right)$. Since $r_{T} \gg w$, $K_{i, B}^{+ \pm}$is non-zero only for a small solid angle $\Omega_{w}$. The size of the solid angle can be estimated as $\Omega_{w} \sim w^{2} / 4 r_{T}^{2}$ and $K_{i, B}^{+ \pm}$within that small solid angle can be estimated as a constant value $K_{i, B}^{+ \pm}\left(\mathbf{r}_{T}, \hat{\mathbf{r}}_{T}\right)$. The values of $\Gamma_{i, B}^{j+ \pm}$ and $\left\langle\mathbf{F}_{i, B}^{+ \pm}\left(\mathbf{r}_{T}\right)\right\rangle$, then, can be estimated as

$$
\begin{gathered}
\Gamma_{i, B}^{j+ \pm} \sim K_{i, B}^{+ \pm}\left(\mathbf{r}_{T}, \hat{\mathbf{r}}_{T}\right) \cdot \frac{w^{2}}{4 r_{T}^{2}} \cdot \hat{\mathbf{r}}_{T}, \\
\left\langle\mathbf{F}_{i, B}^{+ \pm}\left(\mathbf{r}_{T}\right)\right\rangle \sim f_{i, B} \cdot K_{i, B}^{+ \pm}\left(\mathbf{r}_{T}, \hat{\mathbf{r}}_{T}\right) \cdot \frac{w^{2}}{4 r_{T}^{2}} \cdot \hat{\mathbf{r}}_{T} .
\end{gathered}
$$

Since $K_{i, B}^{+ \pm}$is within $\Omega_{w}$ approximated by a constant, we can use $\Gamma_{2, B}^{k l++}$ given by equations (125) and (126) to calculate $\Gamma_{i, B}^{k l+ \pm}$. In transfer $I_{2}$ the direction $\hat{\mathbf{r}}_{T}$ is along $+\mathbf{z}$ axis while in the herein examined transfers $\hat{\mathbf{r}}_{T}$ has a general direction given by equation (103). Moreover, within $\Omega_{w}$ it is $K_{2, B}^{++}\left(r_{T} \cdot \hat{\mathbf{z}}, \hat{\mathbf{z}}\right)=1 / 4 \pi$, while here we use $K_{i, B}^{+ \pm}\left(\mathbf{r}_{T}, \hat{\mathbf{r}}_{T}\right)$. Therefore, we need to rotate and rescale $\Gamma_{2, B}^{k l++}$ to get $\Gamma_{i, B}^{k l+ \pm}$,

$$
\Gamma_{i, B}^{k l+ \pm} \sim \frac{K_{i, B}^{+ \pm}\left(\mathbf{r}_{T}, \hat{\mathbf{r}}_{T}\right)}{K_{2, B}^{++}\left(r_{T} \cdot \hat{\mathbf{z}}, \hat{\mathbf{z}}\right)} \cdot \mathscr{R} \cdot \Gamma_{2, B}^{k l++} \cdot \mathscr{R}^{-1},
$$

where $\mathscr{R}=\mathscr{R}\left(\vartheta_{T}, \varphi_{T}\right)$ is the rotation matrix that rotates first by angle $\vartheta_{T}$ within plane $z x$ and then by angle $\varphi_{T}$ within plane $x y$. When we explicitly express $\Gamma_{i, B}^{k l+ \pm}$ for $r_{T} \gg w$, we get

$$
\Gamma_{i, B}^{k l+ \pm} \sim 4 \pi \cdot K_{i, B}^{+ \pm}\left(\mathbf{r}_{T}, \hat{\mathbf{r}}_{T}\right) \cdot \Gamma_{2, B,(r o t)}^{k l++}
$$

where $\Gamma_{2, B,(\text { rot })}^{k l++}=\mathscr{R} \cdot \Gamma_{2, B}^{k l++} \cdot \mathscr{R}^{-1}$, or explicitly

$$
\begin{aligned}
& \Gamma_{2, B,(r o t)}^{k l++}= \\
& \frac{w^{2}}{4 r_{T}^{2}} \cdot\left(\begin{array}{ccc}
s_{\vartheta}^{2} c_{\varphi}^{2}+\frac{w^{2}}{4 r_{T}^{2}}\left(c_{\vartheta}^{2} c_{\varphi}^{2}+s_{\varphi}^{2}-s_{\vartheta}^{2} c_{\varphi}^{2}\right) & s_{\vartheta}^{2} s_{\varphi} c_{\varphi}\left(1-\frac{w^{2}}{2 r_{T}^{2}}\right) & s_{\vartheta} c_{\vartheta} c_{\varphi}\left(1-\frac{w^{2}}{2 r_{T}^{2}}\right) \\
s_{\vartheta}^{2} s_{\varphi} c_{\varphi}\left(1-\frac{w^{2}}{2 r_{T}^{2}}\right) & s_{\vartheta}^{2} s_{\varphi}^{2}+\frac{w^{2}}{4 r_{T}^{2}}\left(c_{\vartheta}^{2} s_{\varphi}^{2}+c_{\varphi}^{2}-s_{\vartheta}^{2} s_{\varphi}^{2}\right) & s_{\vartheta} c_{\vartheta} s_{\varphi}\left(1-\frac{w^{2}}{2 r_{T}^{2}}\right) \\
s_{\vartheta} c_{\vartheta} c_{\varphi}\left(1-\frac{w^{2}}{2 r_{T}^{2}}\right) & s_{\vartheta} c_{\vartheta} s_{\varphi}\left(1-\frac{w^{2}}{2 r_{T}^{2}}\right) & c_{\vartheta}^{2}+\frac{w^{2}}{4 r_{T}^{2}}\left(s_{\vartheta}^{2}-c_{\vartheta}^{2}\right)
\end{array}\right),
\end{aligned}
$$


where $s_{\vartheta}=\sin \vartheta_{T}, c_{\vartheta}=\cos \vartheta_{T}, s_{\varphi}=\sin \varphi_{T}$ and $c_{\varphi}=\cos \varphi_{T}$.

Information transfers $I_{6}$ and $I_{14}$ differ from transfers $I_{22}$ and $I_{30}$ in their definition of $\mathbf{r}_{T}$. In the case of $I_{6}$ and $I_{14}, \mathbf{r}_{T}$ is defined by

$$
\mathbf{r}_{T}=\mathbf{x}_{B}\left(\tau_{E}\right)-\mathbf{x}_{A}\left(\tau_{A}\right) .
$$

On the other hand, in the case of $I_{22}$ and $I_{30}, \mathbf{r}_{T}$ is defined by

$$
\mathbf{r}_{T}=\mathbf{x}_{A}\left(\tau_{E}\right)-\mathbf{x}_{A}\left(\tau_{A}\right),
$$

that is it can be written as

$$
\mathbf{r}_{T}=\left\langle\mathbf{v}_{A}\left(\tau_{A B}\right)\right\rangle \cdot\left(\tau_{B}-\tau_{A}\right) .
$$

The information transfers $I_{22}$ and $I_{30}$, therefore, highly depend on the behavior of particle A.

7.21 Information transfers with $\tau_{0}<\tau_{A}=\tau_{E}$

Information transfers $I_{4}, I_{12}, I_{20}$ and $I_{28}$ satisfy $\tau_{0}<\tau_{A}=\tau_{E}$. Let vector $\mathbf{z}, \mathbf{r}_{S}$ and $\mathbf{r}_{T}$ be defined as

$$
\begin{aligned}
\mathbf{z} & =\mathbf{x}_{B}\left(\tau_{A}\right)-\mathbf{x}_{A}\left(\tau_{A}\right), \\
\mathbf{r}_{S}=\mathbf{r}_{T} & =\mathbf{x}_{T}\left(\tau_{E}\right)-\mathbf{x}_{S}\left(\tau_{0}\right),
\end{aligned}
$$

respectively. In transfers $I_{4}$ and $I_{12}$ with $T=B$, mediatorino $m$ is at time $\tau_{A}$ co-local with particle $B$, and thus generally not co-local with particle $A$. The value of $\Delta \mathbf{q}$ is, therefore, determined by law 1-(b). In the transfers with $\tau_{0}<\tau_{A}=\tau_{E}$, the value of $\Delta \mathbf{q}$ is taken from particle $A$ only if mediatorino $m$ hits particle $T$. The probability of hitting randomly located particle $T$ by mediatorino $m$ with radius $w$ is

$$
P_{w}\left(\mathbf{r}_{S}\right)=\Omega_{w}\left(\mathbf{r}_{S}\right) / 4 \pi,
$$

where $\Omega_{w}$ for $r_{S} \leq w$ satisfies $\Omega_{w}\left(\mathbf{r}_{S}\right)=4 \pi$ and for $r_{S}>w$ it is given by equation (108). Therefore, we can immediately write that for $i \in\{4,12\}$

$$
K_{i, A}^{+}\left(\mathbf{r}_{S}, \vartheta, \varphi\right)=P_{w}\left(\mathbf{r}_{S}\right) \cdot \delta_{d}(\vartheta-\pi, \varphi-\pi) .
$$

The corresponding $\Gamma_{i, A}^{j+}$ and $\Gamma_{i, A}^{k l+}$ are given by

$$
\begin{gathered}
\Gamma_{i, A}^{j+}=P_{w}\left(\mathbf{r}_{S}\right) \cdot(0,0,-1)^{T}, \\
\Gamma_{i, A}^{k l+}=P_{w}\left(\mathbf{r}_{S}\right) \cdot\left(\begin{array}{lll}
0 & 0 & 0 \\
0 & 0 & 0 \\
0 & 0 & 1
\end{array}\right) .
\end{gathered}
$$

The mean force on particle $A$ can for $i \in\{4,12\}$ and $r_{S}>0$ be expressed as

$$
\left\langle\mathbf{F}_{i, A}^{+}\left(\mathbf{r}_{S}\right)\right\rangle=-f_{i, A} \cdot P_{w}\left(\mathbf{r}_{S}\right) \cdot \hat{\mathbf{z}},
$$

where $f_{i, A}$ is given by equation (41) with $\left\langle v_{i, A}\right\rangle$ given by equation (111).

In transfers $I_{20}$ and $I_{28}$ with $T=A$, mediatorino $m$ is at time $\tau_{A}$ co-local with particle $A$, and thus $\Delta \mathbf{q}$ is always determined by law 1-(a). For $i \in\{20,28\}$, we obtain

$$
K_{i, A}^{+}\left(\mathbf{r}_{S}, \vartheta, \varphi\right)=P_{w}\left(\mathbf{r}_{S}\right) / 4 \pi .
$$


The corresponding $\Gamma_{i, A}^{j+}$ and $\Gamma_{i, A}^{k l+}$ are given by

$$
\begin{aligned}
\Gamma_{i, A}^{j+} & =(0,0,0)^{T}, \\
\Gamma_{i, A}^{k l+} & =P_{w}\left(\mathbf{r}_{S}\right) \cdot\left(\begin{array}{ccc}
1 / 3 & 0 & 0 \\
0 & 1 / 3 & 0 \\
0 & 0 & 1 / 3
\end{array}\right) .
\end{aligned}
$$

The mean force on particle $A$ can for $i \in\{20,28\}$ be expressed as

$$
\left\langle\mathbf{F}_{i, A}^{+}\left(\mathbf{r}_{S}\right)\right\rangle=\mathbf{0} \text {. }
$$

In all the four cases, $\Delta \mathbf{q}$ is at time $\tau_{A}$ transferred from particle $A$ to particle $B$ directly via an entanglement. Therefore, for $i \in\{4,12,20,28\}$,

$$
\begin{aligned}
K_{i, B}^{+ \pm}\left(\mathbf{r}_{T}, \vartheta, \varphi\right) & =K_{i, A}^{+}\left(\mathbf{r}_{S}, \pi-\vartheta, \pi+\varphi\right), \\
\Gamma_{i, B}^{j+ \pm} & =-\Gamma_{i, A}^{j+}, \\
\Gamma_{i, B}^{k l+ \pm} & =\Gamma_{i, A}^{k l+} \\
\left\langle\mathbf{F}_{i, B}^{+ \pm}\left(\mathbf{r}_{T}\right)\right\rangle & =-\left\langle\mathbf{F}_{i, A}^{+}\left(\mathbf{r}_{S}\right)\right\rangle .
\end{aligned}
$$

This concludes our investigation of directional distributions $K_{i, A}$ and $K_{i, B}$ of the studied information transfers.

\subsection{Transformations and symmetries}

Symmetries are important properties of each information transfer. We distinguish two types of symmetries, fundamental and emergent. By the fundamental symmetry, we mean a symmetry in a transfer of $\triangle q$ from particle $A$ to particle $B$. By the emergent symmetry we mean any non-fundamental symmetry which emerges when we combine several transfers. It can be a combination of different paths, such as a transfer of information from particle $A$ to particle $B$ with a transfer of information from particle $B$ to particle $A$ within the same transfer $I_{i}$, or it can be a combination of several different information transfers. Since transfers $I_{2}, I_{4}$, $I_{6}$ and $I_{28}$ do not require any entanglement between $A$ and $B$, each of them may naturally demonstrate emergent symmetries. The other information transfers require $\varepsilon(R, \bar{R})$ in order to work, and thus they may demonstrate emergent symmetries if there is also $\varepsilon(\bar{R}, R)$. In the following text, if not said otherwise, we investigate only the fundamental symmetries of information transfers.

\subsubsection{Q transformation-reverting sign of $\triangle \mathbf{q}$}

For all the information transfers we have expressed only $K_{i}^{+}$. The sign + specifies that $\triangle \mathbf{q}$ at time $\tau_{A}$ points away from particle $A$. Now we express $K_{i}^{-}$with $\triangle \mathbf{q}$ pointing toward particle $A$. The value $K_{i}^{-}$can be obtained from $K_{i}^{+}$by transformation

$$
\triangle \mathbf{q} \stackrel{Q}{\rightarrow}-\triangle \mathbf{q}
$$

This transformation will be called $Q$ transformation, and quantities of information transfers being symmetric with respect to this transformation will be called $Q$-symmetric. Since the reversion of $\Delta \mathbf{q}$ to $-\triangle \mathbf{q}$ is within $K_{i}(\mathbf{r}, \hat{\mathbf{e}})$ equivalent to the change of the direction of $\hat{\mathbf{e}}$ to its opposite, for particle $A$ we have 


$$
\begin{aligned}
K_{i, A}^{-}\left(\mathbf{r}_{S}, \hat{\mathbf{e}}\right) & =K_{i, A}^{+}\left(\mathbf{r}_{S},-\hat{\mathbf{e}}\right), \\
\Gamma_{i, A}^{j-}\left(\mathbf{r}_{S}\right) & =-\Gamma_{i, A}^{j+}\left(\mathbf{r}_{S}\right), \\
\Gamma_{i, A}^{k l-}\left(\mathbf{r}_{S}\right) & =\Gamma_{i, A}^{k l+}\left(\mathbf{r}_{S}\right) .
\end{aligned}
$$

The second sign in the superscript of $K_{i, B}^{ \pm \pm}$specifies the orientation of $\mathbf{v}_{m}\left(\tau_{A B}\right)$ which is independent of the orientation of $\triangle \mathbf{q}$. It means that the transformation $\triangle \mathbf{q} \rightarrow-\triangle \mathbf{q}$ does not change the second sign. Thus, for particle $B$ we have

$$
\begin{aligned}
K_{i, B}^{- \pm}\left(\mathbf{r}_{T}, \hat{\mathbf{e}}\right) & =K_{i, B}^{+ \pm}\left(\mathbf{r}_{T},-\hat{\mathbf{e}}\right), \\
\Gamma_{i, B}^{j- \pm}\left(\mathbf{r}_{T}\right) & =-\Gamma_{i, B}^{j+ \pm}\left(\mathbf{r}_{T}\right), \\
\Gamma_{i, B}^{k l- \pm}\left(\mathbf{r}_{T}\right) & =\Gamma_{i, B}^{k l+ \pm}\left(\mathbf{r}_{T}\right) .
\end{aligned}
$$

Some information transfers have $Q$-symmetric $K_{i, A}$ and some have $Q$-symmetric $K_{i, B}$. In transfers $I_{1}, I_{2}, I_{3}, I_{9}, I_{10}, I_{11}\left(\tau_{0}=\tau_{A}, S=A\right), I_{20}$ and $I_{28}\left(\tau_{0}<\tau_{A}=\tau_{E}, T=A\right)$ particle $A$ is at time $\tau_{A}$ co-local with mediatorino $m$, and thus $\mathbf{x}_{A}^{c}\left(\tau_{A}\right)=\mathbf{x}_{m}^{c}\left(\tau_{A}\right)$. Law 1 , then, guarantees point-symmetric $K_{i, A}$, and thus we can immediately write for the transfers $I_{i}$ with $i \in\{1,2,3,9,10,11,20,28\}$ :

$$
\begin{aligned}
K_{i, A}^{-}\left(\mathbf{r}_{S}, \hat{\mathbf{e}}\right) & =K_{i, A}^{+}\left(\mathbf{r}_{S}, \hat{\mathbf{e}}\right), \\
\Gamma_{i, A}^{j-}\left(\mathbf{r}_{S}\right) & =\Gamma_{i, A}^{j+}\left(\mathbf{r}_{S}\right)=\mathbf{0}, \\
\Gamma_{i, A}^{k l-}\left(\mathbf{r}_{S}\right) & =\Gamma_{i, A}^{k l+}\left(\mathbf{r}_{S}\right),
\end{aligned}
$$

that is transfers $I_{1}, I_{2}, I_{3}, I_{9}, I_{10}, I_{11}, I_{20}$ and $I_{28}$ have $Q$-symmetric $K_{i, A}$. Moreover, in transfers $I_{1}, I_{3}, I_{9}, I_{11}, I_{20}$ and $I_{28}$, transferring $\triangle \mathbf{q}$ to particle $B$ always via at least one entanglement implies $K_{i, B}\left(\mathbf{r}_{T}, \hat{\mathbf{e}}\right)=K_{i, A}\left(\mathbf{r}_{S},-\hat{\mathbf{e}}\right)$. Therefore, for $i \in\{1,3,9,11,20,28\}$ :

$$
\begin{aligned}
K_{i, B}^{- \pm}\left(\mathbf{r}_{T}, \hat{\mathbf{e}}\right) & =K_{i, B}^{+ \pm}\left(\mathbf{r}_{T}, \hat{\mathbf{e}}\right), \\
\Gamma_{i, B}^{j- \pm}\left(\mathbf{r}_{T}\right) & =\Gamma_{i, B}^{j+ \pm}\left(\mathbf{r}_{T}\right)=\mathbf{0}, \\
\Gamma_{i, B}^{k l- \pm}\left(\mathbf{r}_{T}\right) & =\Gamma_{i, B}^{k l+ \pm}\left(\mathbf{r}_{T}\right) .
\end{aligned}
$$

In other words, information transfers $I_{1}, I_{3}, I_{9}, I_{11}, I_{20}$ and $I_{28}$ have $Q$-symmetric $K_{i, B}$.

\subsubsection{V transformation-reverting sign of $\mathbf{v}_{m}\left(\tau_{A B}\right)$}

The role of $\mathbf{v}_{m}\left(\tau_{A B}\right)$ is to propagate mediatorinos during time interval $\left(\tau_{A}, \tau_{B}\right)$ through space. Since for a given $\triangle \mathbf{q}$ there are two possible directions of $\mathbf{v}_{m}\left(\tau_{A B}\right)$, parallel and antiparallel to $\triangle \mathbf{q}$, we can ask about the symmetry of information transfers with respect to

$$
\mathbf{v}_{m}\left(\tau_{A B}\right) \stackrel{V}{\rightarrow}-\mathbf{v}_{m}\left(\tau_{A B}\right),
$$

hereafter called $V$ transformation. We already know that $K_{i, A}$ is not influenced by $\mathbf{v}_{m}\left(\tau_{A B}\right)$ because $K_{i, A}$ describes the distribution of a change of $\mathbf{q}$ on particle $A$ at time $\tau_{A}$, while $\mathbf{v}_{m}\left(\tau_{A B}\right)$ moves mediatorino $m$ during time interval $\left(\tau_{A}, \tau_{B}\right)$, which is later than at time $\tau_{A}$. Consequently, all the information transfers have $V$-symmetric $K_{i, A}$. It is expressed in the way that $K_{i, A}$ never has the second superscript sign.

Now, let us focus on $K_{i, B}$. In the information transfers with $\tau_{A}=\tau_{B}$ there is no $\tau_{A B}$, and consequently $V$ transformation does not affect $K_{i, B}$ in these transfers. We can, therefore, write that for $i \in\{1,4,5,9,12,13,17,20,21,25,28,29\}$ : 


$$
K_{i, B}^{ \pm-}\left(\mathbf{r}_{T}, \hat{\mathbf{e}}\right)=K_{i, B}^{ \pm+}\left(\mathbf{r}_{T}, \hat{\mathbf{e}}\right) .
$$

In total, the transfers with $\tau_{A}=\tau_{B}$ have $V$-symmetric both $K_{i, A}$ and $K_{i, B}$. Now, let us ask about $V$ symmetry only in the transfers with $\tau_{A}<\tau_{B}$. We exclude transfers $I_{2}, I_{3}, I_{10}$ and $I_{11}$ because they do not have $s_{v}=-1$, and thus $V$ transformation has no good meaning in these transfers. In the case of the transfers with $\tau_{A}<\tau_{B}<\tau_{E}, \Delta \mathbf{q}$ is transferred to particle $B$ via one or two entanglements. Consequently, $K_{i, B}$ is independent of the location of mediatorino $m$ at time $\tau_{B}$, and thus $K_{i, B}$ is also independent of velocity $\mathbf{v}_{m}\left(\tau_{A B}\right)$ which moved the mediatorino to that location. In total, the transfers with $\tau_{A}<\tau_{B}<\tau_{E}$ have $V$-symmetric $K_{i, B}$, or equivalently, for $i \in\{7,15,19,23,27,31\}$ :

$$
K_{i, B}^{ \pm-}\left(\mathbf{r}_{T}, \hat{\mathbf{e}}\right)=K_{i, B}^{ \pm+}\left(\mathbf{r}_{T}, \hat{\mathbf{e}}\right) .
$$

The remaining information transfers, that is transfers $I_{6}, I_{14}, I_{18}, I_{22}, I_{26}$ and $I_{30}$, are the only transfers that may violate $V$-symmetry in $K_{i, B}$.

Directional distribution $K_{i}$ is not the only thing that can be affected by $V$ transformation. $V$ transformation is purely spatial, and thus it can also affect the distribution of mediatorinos in space. The distribution of mediatorinos in space directly depends on the distribution of the velocities of mediatorinos. Let $V_{i, m}^{ \pm}(\vartheta, \varphi, t)$ be the distribution of the velocities of mediatorinos at time $t$, provided the sign in the superscript is equal to the sign of $s_{v}$. Since $\triangle \mathbf{q}$ and $\mathbf{v}_{m}\left(\tau_{A B}\right)$ are either parallel or antiparallel to each other, for $t \in\left(\tau_{A}, \tau_{B}\right)$ we can immediately write

$$
V_{i, m}^{ \pm}\left(\vartheta, \varphi, \tau_{A B}\right)= \begin{cases}K_{i, A}^{ \pm}(\vartheta, \varphi), & \text { if } s_{q} s_{v}=1, \\ K_{i, A}^{\mp}(\vartheta, \varphi), & \text { if } s_{q} s_{v}=-1 .\end{cases}
$$

We are interested in the transfers with $\tau_{A}<\tau_{B}$ because during time interval $\left(\tau_{A}, \tau_{B}\right)$ mediatorinos are real and perhaps detectable. Mediatorinos are detectable at least for transfers $I_{2}$ and $I_{6}$, which not only satisfy $\tau_{A}<\tau_{B}$, but also their mediatorinos can finish on a yet unknown particle $T$, that is on a particle of a detector. We do not go into further details. For now, it is sufficient to keep in mind that asymmetry with respect to $V$-transformation may be measurable via the distribution of mediatorinos in space which depends on $V_{i, m}$.

\subsubsection{P transformation-reverting spatial axes}

One of the most important symmetries is symmetry with respect to parity transformation. Parity transformation, $P$, can be written as

$$
\mathbf{x} \stackrel{P}{\rightarrow}-\mathbf{x}, \quad \mathbf{y} \stackrel{P}{\rightarrow}-\mathbf{y}, \quad \mathbf{z} \stackrel{P}{\rightarrow}-\mathbf{z} .
$$

Location $\mathbf{r}$ is a spatial vector, and thus is transformed as

$$
\mathbf{r} \stackrel{P}{\rightarrow}-\mathbf{r} .
$$

On the other hand, a specific quantity $\mathbf{q}$ (with direction $\mathbf{e}$ for $\Delta \mathbf{q}$ ) can be either vectorial or pseudovectorial. If $\mathbf{q}$ is a vector, then the parity transformation reverts the orientation of $\triangle \mathbf{q}$. That is, for a vectorial $\mathbf{q}$ and all $i \in \mathbb{F}$ we get

$$
\begin{aligned}
K_{i, A}^{ \pm P}(\mathbf{r}, \hat{\mathbf{e}}) & =K_{i, A}^{ \pm}(-\mathbf{r},-\hat{\mathbf{e}}), \\
K_{i, B}^{ + \pm P}(\mathbf{r}, \hat{\mathbf{e}}) & =K_{i, B}^{+ \pm}(-\mathbf{r},-\hat{\mathbf{e}}), \\
K_{i, B}^{ - \pm P}(\mathbf{r}, \hat{\mathbf{e}}) & =K_{i, B}^{- \pm}(-\mathbf{r},-\hat{\mathbf{e}}) .
\end{aligned}
$$


If $\mathbf{q}$ is a pseudovector, then the parity transformation does not affect the orientation of $\Delta \mathbf{q}$,

$$
\begin{aligned}
K_{i, A}^{ \pm P}(\mathbf{r}, \hat{\mathbf{e}}) & =K_{i, A}^{ \pm}(-\mathbf{r}, \hat{\mathbf{e}}), \\
K_{i, B}^{ + \pm P}(\mathbf{r}, \hat{\mathbf{e}}) & =K_{i, B}^{+ \pm}(-\mathbf{r}, \hat{\mathbf{e}}), \\
K_{i, B}^{ - \pm P}(\mathbf{r}, \hat{\mathbf{e}}) & =K_{i, B}^{- \pm}(-\mathbf{r}, \hat{\mathbf{e}}) .
\end{aligned}
$$

The distribution of mediatorinos in space depends on $V_{i, m}\left(\tau_{A B}\right)$, which is the distribution of the velocities of mediatorinos during time interval $\left(\tau_{A}, \tau_{B}\right)$. Since velocity $\mathbf{v}_{m}\left(\tau_{A B}\right)$ is a vector, parity transformation reverts its orientation. The distribution of the velocities of mediatorinos is, therefore, transformed as

$$
V_{i, m}^{ \pm P}\left(\hat{\mathbf{e}}, \tau_{A B}\right)=V_{i, m}^{ \pm}\left(-\hat{\mathbf{e}}, \tau_{A B}\right) .
$$

This, along with a psuedovectorial $\triangle q$, can be used to reveal asymmetry with respect to parity transformation.

\subsubsection{T transformation-reverting flow of time}

Another important symmetry is the symmetry with respect to $T$ transformation, the transformation which reverts the flow of time. Postulate 2 says that time flows only in one direction, and the order of events plays an important role in deciding how each information transfer works and what minimal set of entanglements it requires. If time flowed in the opposite direction, most information transfers could not do all their steps in the reversed order, and consequently they could not transfer information $\triangle q$ from particle $B$ to particle $A$. Firstly, because in the transfers with $T=\bar{R}$ and $\varepsilon(m, R)$ there is no way to create at time $\tau_{E}$ mediatorino $m$ with entanglement $\varepsilon(m, R)$. This is because particle $\bar{R}$ does not know the identity of particle $R$. It comes from no requirement for entanglement $\varepsilon(\bar{R}, R)$, that is even if the entanglement existed, it would not be used in the transfer. Secondly, in the information transfers which require $\varepsilon(R, \bar{R})$, the reverting the flow of time implies that mediatorino $m$ must go from a specific particle $T$ to a specific particle $S$. The problem is that although in most transfers the mediatorino checks for the presence of specific particle $T$, it never checks for the presence of specific particle $S$. There is, therefore, no mechanism which would guarantee that mediatorino $m$ finished within the reversed time its journey on the specific particle $S$. Transfers $I_{4}, I_{6}$ and $I_{28}$, which require $\varepsilon(m, R)$, face the same problem. Mediatorino $m$ must within the reversed time finish on the specific particle $S=R$. All the above-mentioned information transfers, therefore, cannot demonstrate the fundamental time symmetry. That is, if they ever demonstrate time symmetry, it must be an emergent time symmetry. On the other hand, the remaining transfer $I_{2}$ does not require any entanglement. If it also does not require any specific particle $S$ for the interaction, then it is the only transfer that demonstrates fundamental time symmetry.

\subsection{Types of information transfers}

Information transfers can be grouped by forces they produce. We know five types of forces and each of them will be denoted by a letter: $Z$ means a zero average force, $C$ means a constant force, $A$ means an asymptotically free force, $S$ means a speed-dependent force, and finally $L$ means a force based on the co-locality, i.e., $\langle F\rangle \propto 1 / r^{d-1}$. Since the force on particle $A$ is not always the same as the force on particle $B$, the type of an information transfer will be represented by two letters, the first letter specifying the type of the force on particle $A$ and the second letter specifying the type of the force on particle $B$. Two same letters indicate that 
$\triangle \mathbf{q}$ is transferred to particle $B$ via one or more entanglements without any loss. On the other hand, if the letters are not the same, then the second letter is always $L$ indicating that the co-locality plays an important role in the force on particle $B$. For example, $I_{2}$ is a $Z L$-type information transfer because $\left\langle\mathbf{F}_{2, A}\right\rangle$ is a zero average force (equation (60)) and $\left\langle\mathbf{F}_{2, B}\right\rangle$ is a force based on the co-locality (equation (127)). The type of every fundamental information transfer is shown in the periodic table of information transfers, in table 3 . We can see there that 28 fundamental information transfers belong to 10 types of transfers.

\section{Fundamental interactions}

Let us examine whether the fundamental interactions we experience in our Universe can be represented by at least one information transfer.

\subsection{Electromagnetic scattering}

\subsubsection{Force between every two charged particles}

Electromagnetic scattering works between every two electrically charged particles, no matter how many particles are present. On the other hand, information transfers requiring entanglement $\varepsilon(R, \bar{R})$ do not work between every two particles when the number of interacting particles exceeds $2 N_{\varepsilon}^{\max }+1$. It means that such information transfers cannot represent electromagnetic scattering. The only information transfers able to do so are those which do not require entanglement $\varepsilon(R, \bar{R})$ in order to work, that is transfers $I_{2}, I_{4}, I_{6}$ and $I_{28}$.

\subsubsection{Attractive and repulsive force}

Electromagnetic force can be attractive or repulsive depending on the electric charge of the interacting particles. Since information transfer $I_{28}$ always produces a zero force, that is $\left\langle\mathbf{F}_{28, A}\right\rangle=\left\langle\mathbf{F}_{28, B}\right\rangle=\mathbf{0}$, it is neither attractive nor repulsive. The immediate consequence is that transfer $I_{28}$ cannot represent the electromagnetic force.

Let us assume both variants, attractive force and repulsive force, can be achieved by a single mediator, that is there are no two types of mediator such that one causes only an attractive force and the other causes only a repulsive force. Whether electromagnetic force between particles $A$ and $B$ is attractive or repulsive depends on the sign of the electric charge of the particles, and relates to the transferred momentum $\triangle \mathbf{p}$, which, like any other transferred conserved quantity, is determined at time $\tau_{A}$. It means that at time $\tau_{A}$ the mediator must know the sign of the electric charge of particle $B$ in order to determine the orientation of $\triangle \mathbf{p}$ correctly.

Information transfers $I_{2}$ and $I_{6}$ work in the way that they do not rely on entanglement $\varepsilon(M, B)$ nor $\varepsilon(A, B)$. It means that even if these entanglements existed, the transfers would not use them. A consequence is that mediator $M$ never finds out the value of the electric charge of particle $B$ non-locally, it always finds out the value locally. However, in these transfers, the mediator is co-local with particle $B$ only at time $\tau_{B}$. Since $\tau_{B}>\tau_{A}$, the mediator finds out the sign of the electric charge of particle $B$ later than it is needed for the determination of $\triangle \mathbf{p}$. Moreover, postulate 2 forbids mediator $M$ from going backward in time from $\tau_{B}$ to $\tau_{A}$ and using there the value of the charge. In total, information transfers $I_{2}$ and $I_{6}$ do not guarantee an attractive/repulsive force based on the electric charge of particle $B$, and thus the electromagnetic scattering cannot be represented by $I_{2}$ nor by $I_{6}$. 


\subsubsection{Electromagnetic scattering is $I_{4}$}

The remaining information transfer $I_{4}$ is the only transfer able to exhibit force working between every two electrically charged particles and depending on the sign of their charge. It means that electromagnetic scattering can only be represented by $I_{4}$, the transfer which satisfies $\tau_{0}<\tau_{A}=\tau_{B}=\tau_{E}$ and $R=S=A$, and which requires entanglement $\varepsilon\left(M_{4}, A\right)$ in order to work. Let us mention several consequences.

First of all, the value $\Delta q$ is determined at time $\tau_{A}$, that is at the end of transfer $I_{4}$. It means that $\Delta q$ does not exist until the last moment of the transfer. This is a very important quantum property saying that during transfer $I_{4}$ the value $\Delta q$ is not stored in any hidden variable. It also means that mediator $M_{4}$ is always a virtual.

Directional distribution of electric force is, due to equation (164), $K_{4} \propto \delta_{d}(\vartheta, \varphi)$. It means that, except for the sign, the force can have only one direction. Thus, in the calculation of the average force $\left\langle\mathbf{F}_{4, A}\right\rangle$ and $\left\langle\mathbf{F}_{4, B}\right\rangle$ by equations (40) and (37), no information is lost. In other words, electromagnetic interaction between two point particles can be fully described by a vector theory. Moreover, due to equation (24), the direction of the average force satisfies

$$
\left\langle\mathbf{F}_{4, B}\left(\tau_{A}\right)\right\rangle \| \mathbf{x}_{B}\left(\tau_{A}\right)-\mathbf{x}_{A}\left(\tau_{A}\right) .
$$

It means that the force on particle $B$ points along the line going through $\mathbf{x}_{A}\left(\tau_{A}\right)$, the current location of particle $A$, rather than $\mathbf{x}_{A}\left(\tau_{0}\right)$, the location of particle $A$ at the moment it emitted the mediator. This result, which is known already in classical electrodynamics [35], is usually explained in the way that the direction of the electric force is due to the length contraction along the direction of the movement of particle $A$, that is it is a consequence of special relativity. This may indicate that special relativity is already included in the model and we do not need to postulate it explicitly.

\subsection{Strong scattering}

\subsubsection{Interaction between color particles}

It is usually said that strong interaction works between every two color particles. However, the groups of color particles held together by gluons and usually seen in todays' experiments are mesons and baryons, that is pairs and triplets of valence quarks. There has also been found evidence for tetraquarks and pentaquarks [36-39], but they are under an active investigation, and we still do not know much about their dynamics. In other words, our established knowledge is limited to groups of no more than three color particles. For the minimal possible value of $N_{\varepsilon}^{\max }, N_{\varepsilon}^{\max }=1$, it is required to have a group of at least four particles in order to be able to safely determine whether an information transfer can work among all the particles of the group. Therefore, we do not feel safe to assume that every color particle can interact by gluons with every other color particle. Instead, we focus on the properties which are the signatures of strong interaction: confinement and asymptotic freedom.

\subsubsection{Confinement}

In experiments we can see groups of quarks, but no single free quark has ever been observed [10]. The impossibility to separate a single quark, the so-called quark confinement, is caused by the fact that such a separation would require infinite energy, no matter what the initial distance $r_{0}$ between two quarks is, 


$$
\forall r_{0} \in \mathbb{R}:\left|\int_{r_{0}}^{\infty}\left\langle F_{i}(r)\right\rangle d r\right|=\infty .
$$

Information transfer $I_{i}$ cannot demonstrate confinement if none of forces $\left\langle F_{i, A}\right\rangle,\left\langle F_{i, B}\right\rangle$ and $\left|\left\langle F_{i, A}\right\rangle\right|+\left|\left\langle F_{i, B}\right\rangle\right|$ satisfy equation (203). Information transfers $I_{1}, I_{3}, I_{9}, I_{11}, I_{20}$ and $I_{28}$ are on average zero, $\left\langle F_{i, A}\right\rangle=\left\langle F_{i, B}\right\rangle=0$, and thus they never satisfy condition (203). Similarly, transfers $I_{2}$ and $I_{10}$ satisfy $\left\langle F_{i, A}\right\rangle=0$ and $\left\langle F_{i, B}(r)\right\rangle \propto 1 / r^{2}$, and transfers $I_{4}$ and $I_{12}$ satisfy $\left\langle F_{i, A}(r)\right\rangle \propto 1 / r^{2}$ and $\left\langle F_{i, B}(r)\right\rangle \propto 1 / r^{2}$. None of these transfers satisfy condition (203). In total, information transfers $I_{1}$ to $I_{4}, I_{9}$ to $I_{12}, I_{20}$ and $I_{28}$ do not demonstrate confinement, and therefore they cannot represent strong scattering.

\subsubsection{Asymptotic freedom}

Experiments show that quarks inside hadrons in high-energy limit demonstrate the so-called asymptotic freedom [40]. It means that when distance $r$ of two quarks decreases, the color force between the quarks also decreases and eventually vanishes. That is, for every distance $r_{0}>0$ :

$$
0=\lim _{r \rightarrow 0}\left|\left\langle F_{i}(r)\right\rangle\right|<\left|\left\langle F_{i}\left(r_{0}\right)\right\rangle\right|<\lim _{r \rightarrow \infty}\left|\left\langle F_{i}(r)\right\rangle\right| .
$$

Let us examine which information transfers with confinement can also demonstrate asymptotic freedom. We require asymptotic freedom on both particles $A$ and $B$ because a quark in a hadron may play both these roles simultaneously.

In information transfers $I_{5}, I_{7}, I_{13}$, and $I_{15}$, forces $\left\langle F_{i, A}\right\rangle$ and $\left\langle F_{i, B}\right\rangle$ are the functions of the speed of particle $A$ and not the functions of distance $r$ between particle $A$ and particle $B$. Consequently, they cannot systematically satisfy condition (204). Similarly, in information transfers $I_{6}$ and $I_{14}$, force $\left\langle F_{i, A}\right\rangle$ is the function of the speed of particle $A$ and not any function of distance $r$ between particles $A$ and $B$ and $\left\langle F_{i, B}(r)\right\rangle \propto 1 / r^{2}$. These transfers also cannot systematically satisfy condition (204). Hence, information transfers $I_{5}$ to $I_{7}$ and $I_{13}$ to $I_{15}$ do not demonstrate asymptotic freedom, and thus they cannot represent strong scattering. In information transfers $I_{17}, I_{19}, I_{25}$ and $I_{27}$ there is $\left|\lim _{r \rightarrow 0}\left\langle F_{i, A}(r)\right\rangle\right|=f_{i, A}$, and $\left|\lim _{r \rightarrow 0}\left\langle F_{i, B}(r)\right\rangle\right|=f_{i, B}$. Since $f_{i}>0$, these transfers do not satisfy condition (204). It means they also cannot represent strong scattering. In information transfers $I_{18}$ and $I_{26}$, $\left|\lim _{r \rightarrow 0}\left\langle F_{i, A}(r)\right\rangle\right|=f_{i, A} \neq 0$ and $\left\langle F_{i, B}(r)\right\rangle \propto 1 / r^{2}$. Therefore, transfers $I_{18}$ and $I_{26}$ also do not satisfy condition (204), and cannot represent strong scattering. Information transfers $I_{22}$ and $I_{30}$ satisfy asymptotic freedom on particle $A$ but not on particle $B,\left\langle F_{i, B}\right\rangle \propto 1 / r^{2}$. Consequently, these transfers can also be ruled out of the strong scattering candidates.

\subsubsection{Strong scattering candidate transfers}

The only information transfers satisfying both confinement and asymptotic freedom on both particles are transfers $I_{21}, I_{23}, I_{29}$ and $I_{31}$. The question whether all these four information transfers really represent strong scattering we leave open and assume they do. Let us look at some consequences for strongly interacting particles.

Information transfers $I_{21}, I_{23}, I_{29}$ and $I_{31}$ have common features. Firstly, they all satisfy $\tau_{0}<\tau_{A}<\tau_{E}$ and $S=B$. For short distances $r$ between two strongly interacting particles $A$ and $B, r<R$, the total mean force $\left\langle\mathbf{F}_{i}\right\rangle$ is composed of force contributions with every possible direction. That is, every force contribution with a given direction partially or fully cancels out a force contribution with the opposite direction. The smaller the distance $r$ is, the 
better the cancellation is. For $r=0$, the force contributions from all the directions are equal, and thus the total force is zero. This is how asymptotic freedom is demonstrated in strong interaction. The transferring of $\Delta \mathbf{q}$ always via one or two entanglements over an arbitrary distance makes the force between strongly interacting particles not vanish at large distances. This, along with the efficiency of the transfers of $\Delta \mathbf{q}$ via entanglement(s), causes all the mutually interacting particles to be very close to each other, i.e., they are confined. Since strongly interacting particles do not interact with not entangled particles, the proximity of the entangled particles, then, makes strong scattering be seemingly of a short range. This results in the fact that quarks of two hadrons do not interact with each other in the same way as quarks of the same hadron do. The requirement $N_{\varepsilon}^{\max } \leq 2$ for particle $R$ makes strongly interacting particles highly selective in what particles they interact with. This results in forming groups with a small number of strongly interacting particles rather than with a large number of strongly interacting particles. All the candidate information transfers for strong scattering satisfy equations (87) and (99), and thus the average force corresponds to a potential

$$
V(r)= \begin{cases}f \cdot \frac{r^{2}}{3 R}, & \text { if } r \leq R, \\ f \cdot\left(r-R+\frac{R^{2}}{3 r}\right), & \text { if } r>R,\end{cases}
$$

where $f$ and $R$ are $r$-independent values. First of all, this potential behaves linearly at large distances, and thus satisfies Wilson criterion for confinement [41]. Moreover, for $r>R$ the potential has, except for ignorable additive constant $f R$, the same form as Cornell potential [42]

$$
V(r)=\sigma \cdot r+\frac{\kappa}{r}
$$

which is, nowadays, the most promising potential of strong interaction presented in the literature.

There is a subtle difference between strong interaction mediated by transfers $I_{21}, I_{23}, I_{29}$ and $I_{31}$ and conventional strong interaction within current quantum chromodynamics. In the former model, it is possible to have strongly interacting particle $C$ which does not interact strongly with strongly interacting particle $D$ (just because there is no entanglement between $C$ and $D$ ). However, in the latter model every two color particles interact strongly with each other. Only a group of at least two color particles may be neutral in the sense that it does not interact strongly with color particle $D$. Only an experiment can decide which of these models of strong interaction (if any) is realized in our Universe.

\subsection{Weak decay}

\subsubsection{Decay as information transfer}

Information transfers can represent a decay. If an information transfer represents a decay, then it behaves as if it had only the part when $\triangle q$ is taken from particle $A$. It means the part when $\triangle q$ is transferred to particle $B$ is highly suppressed. The very low probability of transferring $\Delta q$ to particle $B$ is the reason why the whole process is not recognized as a transfer, and is considered to be a decay of particle $A$.

The information transfers with $\tau_{B}<\tau_{E}$ have, according to equation (94), a very high probability of transferring $\triangle q$ to particle $B$, and thus they can be ruled out of the candidates 
able to represent a decay. Consequently, the information transfers which can represent a decay satisfy $\tau_{B}=\tau_{E}$.

\subsubsection{Weak decay as two-phase process}

Weak decay is not a point decay of Fermi type, it always has two phases. In the first phase, the intermediate boson is virtual, while in the other phase the products of the decay are real particles.

Information transfers $I_{2}, I_{10}, I_{18}$ and $I_{26}$ satisfy $\tau_{0}=\tau_{A}<\tau_{B}=\tau_{E}$. It means they only have time phase $\left[\tau_{A}, \tau_{B}\right]$ where mediator $M$ is real. These transfers, therefore, behave as a point decay with no characteristic time corresponding to the lifetime of the intermediate boson. In conclusion, transfers $I_{2}, I_{10}, I_{18}$ and $I_{26}$ cannot represent weak decay. Similarly, information transfers $I_{4}, I_{12}, I_{20}$ and $I_{28}$ with timing $\tau_{0}<\tau_{A}=\tau_{B}=\tau_{E}$ have only time phase $\left[\tau_{0}, \tau_{A}\right]$, where mediator $M$ is virtual. It means they do not have any time phase where the products of the decay were real particles. Consequently, transfers $I_{4}, I_{12}, I_{20}$ and $I_{28}$ cannot represent weak decay. So far not ruled out information transfers are $I_{6}, I_{14}, I_{22}$ and $I_{30}$ which satisfy $\tau_{0}<\tau_{A}<\tau_{B}=\tau_{E}$.

\subsubsection{Weak decay as parity violating process}

Weak interaction violates symmetry with respect to parity transformation [43]. The parity violation can be quantified by the longitudinal polarization defined [44] as

$$
P_{l}=\frac{N^{+}-N^{-}}{N^{+}+N^{-}},
$$

where $N^{+}$and $N^{-}$is the number of emitted particles with positive and negative helicity, respectively. Any non-zero value $P_{l}$ means parity violation. The values $N^{+}$and $N^{-}$can be expressed as

$$
\begin{aligned}
& N^{+}=N \int_{0}^{2 \pi} \int_{0}^{\pi / 2} I(\vartheta, \varphi) \sin \vartheta d \vartheta d \varphi, \\
& N^{-}=N \int_{0}^{2 \pi} \int_{0}^{\pi / 2} I(\pi-\vartheta, \pi+\varphi) \sin \vartheta d \vartheta d \varphi,
\end{aligned}
$$

where $N$ is the total number of emitted particles and $I(\vartheta, \varphi)$ is the angular distribution of a product of the decay. The angular distribution $I(\vartheta, \varphi)$ is usually taken in the form [45]

$$
I(\vartheta, \varphi)=(\text { const } .) \cdot(1+\alpha \cos \vartheta) .
$$

This form is reasonable for point processes, but weak decay is not a point process. Thus, instead of using equation (210), we use

$$
I(\vartheta, \varphi)=V_{i, M}\left(\tau_{A B}\right),
$$

$V_{i, M}$ being the directional distribution of the velocities of mediatorinos defined by equation (192). If we align the spin of the system along $z$-axis, we still do not know whether in a concrete situation the spin will be parallel or antiparallel to $\mathbf{z}$ defined by equation (69). That is, we do not know whether we should use $V_{i, M}\left(\vartheta, \varphi, \tau_{A B}\right)$ or $V_{i, M}\left(\pi-\vartheta, \pi+\varphi, \tau_{A B}\right)$ in 
equation (211). Similarly, we do not know whether a concrete process is described by $V_{i, M}^{+}$ or by $V_{i, M}^{-}$. Since all the possible results of $P_{l}$ differ only in the sign, we evaluate the absolute value of $P_{l}$. Let for a moment write $v_{A}=v_{A}\left(\tau_{0 A}\right), v_{M}=v_{M}\left(\tau_{0 A}\right)$ and $R=v_{M} \cdot\left(\tau_{A}-\tau_{0}\right)$. If we calculate $\left|P_{l}\right|$ with the help of equation (207), then for information transfers $I_{6}$ and $I_{14}$ we get

$$
\left|P_{l}\right|= \begin{cases}\frac{v_{A}}{v_{M}}, & \text { if } v_{A} \leq v_{M}, \\ 1, & \text { if } v_{A}>v_{M},\end{cases}
$$

and for information transfers $I_{22}$ and $I_{30}$ we get

$$
\left|P_{l}\right|= \begin{cases}\frac{r_{S}}{R}, & \text { if } r_{S} \leq R \\ 1, & \text { if } r_{S}>R\end{cases}
$$

where $r_{S}$ is the distance of particle $A$ and particle $B$. The speed $v_{M}$ cannot exceed the speed of light and $\tau_{A}-\tau_{0}$ is of the same order as $W^{ \pm}$lifetime. That is, $R \sim 10^{-17} \mathrm{~m}$. Even if particles $A$ and $B$ were very close to each other, such as quarks with the distance of order $r_{S} \sim 10^{-15} \mathrm{~m}$, it is definitely $r_{S}>R$. In total, for most situations information transfers $I_{22}$ and $I_{30}$ predict $\left|P_{l}\right|=1$.

Experiments show $[46,47]$ that in beta decay

$$
\left|P_{l}\right|=\frac{v_{e}}{c},
$$

where $v_{e}$ is the speed of emitted electrons. The experimental result does not depend on any distance. Moreover, the result does not seem to be exactly 1 , especially for slow electrons. Therefore, $I_{22}$ and $I_{30}$ can be ruled out of the candidates able to describe weak decay.

\subsubsection{Weak decay candidate transfers}

The remaining information transfers $I_{6}$ and $I_{14}$ behave as non-point decays with characteristic time $\tau_{A}-\tau_{0}$ corresponding to the intermediate boson lifetime and predict speed-dependent parity violation. Consequently, they are the best candidates for a weak decay. Transfer $I_{6}$ does not require any entanglement between $A$ and $B$ in order to work. So it can work for both entangled and non-entangled particles, that is it can represent a universal weak decay. On the other hand, transfer $I_{14}$ requires $\varepsilon(R, \bar{R})$, and consequently it cannot represent a universal decay. Since the only change of conserved quantities on particle $A$ occurs at time $\tau_{A}$, time $\tau_{A}$ is the time when particle $A$ decays and not time $\tau_{0}$. Transfers $I_{6}$ and $I_{14}$ satisfy $\tau_{0}<\tau_{A}$, and thus their mediator $M$ is during $\left(\tau_{0}, \tau_{A}\right)$ composed of two parts, $m_{1}$ and $m_{2}$. Since the universal computer system is passively optimal, each of the parts survives the time of the decay of particle $A, \tau_{A}$, and the decay of particle $A$ has always three products. They come from $A, m_{1}$ and $m_{2}$. Moreover, since parts $m_{1}$ and $m_{2}$ were before the decay mutually interrelated, we expect two of the products of the decay to be also somehow interrelated.

Equation (212) with $v_{A}>v_{M}$ does not seem to occur in experiments. Therefore, the situation with $v_{A}>v_{M}$ either does not exist (because of kinematical or dynamic reasons) or we have not built a suitable experiment which would induce this condition. On the other hand, equation (212) is for $v_{A} \leq v_{M}$ formally the same as equation (214). Nonetheless, it is important to note that these equations express different things. In order to see it, we need to realize that $v_{A}\left(\tau_{0 A}\right)$ is not the speed of an electron that is emitted in the decay. This is because the electron is created at time $\tau_{A}$ while $v_{A}\left(\tau_{0 A}\right)$ is the speed of particle $A$ during interval $\left(\tau_{0}, \tau_{A}\right)$, that is before particle $A$ decays. For further examination of the speed ratio 


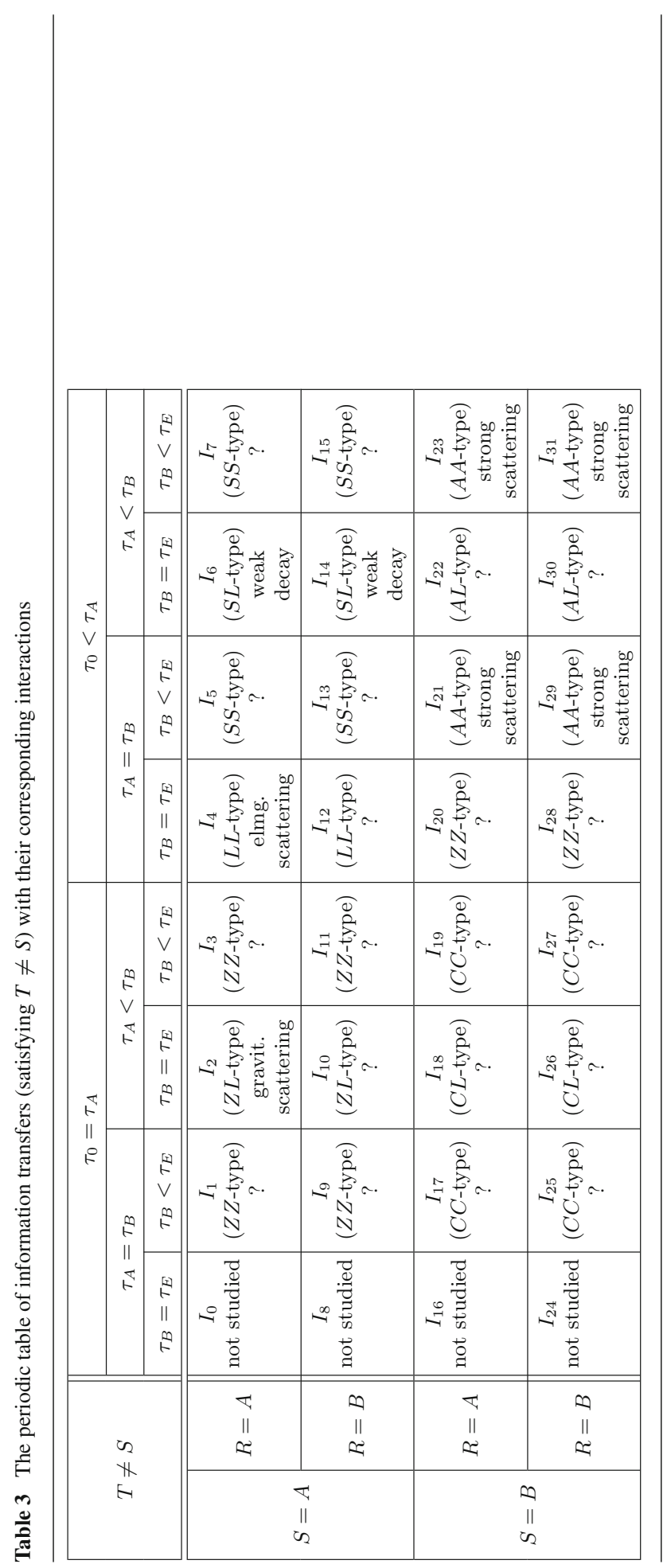


we would need to know exactly what happens at time $\tau_{A}$, that is we would need a definition of momentum, energy, their conservation laws and average decay. Since we still do not have them in the model, we do not try to explain the speed ratio here.

\subsection{Gravity}

\subsubsection{Non-zero force for every particle}

Gravity is assumed to be the interaction by which all matter and energy interact [48]. It means that gravity works between particles $A$ and $B$ even if they are not entangled. Since information transfers $I_{i}$ with $i \in \mathbb{F} \backslash\{2,4,6,28\}$ require entanglement $\varepsilon(R, \bar{R})$ in order to work, they cannot represent gravity. Thus, the only viable candidates for gravity are transfers $I_{2}, I_{4}, I_{6}$ and $I_{28}$.

Gravitational force, though very weak, is systematically non-zero. However, due to equations (171) and (175) transfer $I_{28}$ always satisfies $\left\langle\mathbf{F}_{28, A}\right\rangle=\left\langle\mathbf{F}_{28, B}\right\rangle=\mathbf{0}$. Therefore, we conclude that information transfer $I_{28}$ cannot represent gravity.

Let us assume there exists at least one particle $R$ such that mediators can in no way entangle particle $R$ (for example, because due to incomplete access of the mediators to particle $R$, the self-entanglement of particle $R$ is hidden from the mediators). Information transfers $I_{4}$ and $I_{6}$ require entanglement $\varepsilon(M, R)$ in order to work, but particle $R$ cannot be entangled in that way. It means that particle $R$ cannot interact by transfers $I_{4}$ and $I_{6}$. Since particle $R$, like any other particle, interacts by gravity, transfers $I_{4}$ and $I_{6}$ cannot represent gravity. Note that since particle $R$ cannot interact by transfer $I_{4}$, it must be electrically neutral, and since it cannot interact by transfer $I_{6}$ (and obviously also by $I_{14}$ ), it must be stable with respect to weak decay. In total, if such a non-entangleable neutral stable particle exists (maybe graviton itself is the particle), then the only information transfer able to represent gravity is $I_{2}$.

\subsubsection{Ruling out transfers $I_{4}$ and $I_{6}$}

Information transfers $I_{4}$ and $I_{6}$ can be ruled out of the candidates for gravity even if the assumed non-entangleable particle does not exist.

General relativity shows that gravity between two point-like particles must be described at least by a tensor theory and cannot be reduced to a vector theory without loss of some relevant properties. In information transfer $I_{4}$ between two point-like particles $A$ and $B$ directional distributions $K_{4, A}$ and $K_{4, B}$ are given by equations (164) and (172), respectively. Since both distributions are proportional to directional Dirac delta function $\delta_{d}$, the transfer is purely vectorial. It means that the transfer cannot completely describe tensor-based gravity even in the case of interaction of two point-like particles. Consequently, information transfer $I_{4}$ cannot represent gravity.

Every particle in the Universe must be both the source of gravity (plays the role of particle $A$ ) and the target of gravity (plays the role of particle $B$ ). If there was a type of particles whose particles play in gravity only the role of particle $A$ (and never the role of particle $B$ ), then two particles of this type would not gravitationally interact with each other. Similarly, if there existed a type of particles whose particles play in gravity only the role of particle $B$, then two particles of this type would also not gravitationally interact with each other. The consequence is that the total gravitational force between two particles exerted on of each of them is $\left\langle\mathbf{F}_{A}\right\rangle+\left\langle\mathbf{F}_{B}\right\rangle$. Since within the remaining information transfers $I_{2}$ and $I_{6}$, for $v_{A}=0$ there is $\left\langle\mathbf{F}_{2}\right\rangle=\left\langle\mathbf{F}_{6}\right\rangle$, we need to use $v_{A} \neq 0$ in order to distinguish them. Moreover, since gravity is at large distances attractive, we are interested in the case of $s_{q}=-1$. For small 
speed $v_{A} \ll v_{m}$ and $s_{q}=-1$, we obtain

$$
\left\langle\mathbf{F}_{6, A}^{-}\right\rangle+\left\langle\mathbf{F}_{6, B}^{- \pm}\right\rangle \propto f_{i, A} \frac{2 v_{A}}{3 v_{m}} \hat{\mathbf{z}}+\frac{f_{i, B}}{4 \pi}\left(1 \pm \frac{2 v_{A}}{v_{m}} \cos \vartheta_{T}\right) \frac{w^{2}}{4 r_{T}^{2}} \hat{\mathbf{r}}_{T},
$$

where $\mathbf{z}$ is defined by equation (69). If gravity depended on $v_{A}$ in that way, the first term would lead to an exponential acceleration of particle $A$ in the direction of $+\mathbf{z}$-axis even for small speed $v_{A}$. However, gravity does not depend on the speed of particle $A$ in this way. Therefore, information transfer $I_{6}$ cannot represent gravity. Ruling $I_{6}$ out of the candidates for gravity is also supported by the fact that gravity does not violate parity, however, as we have seen earlier transfer $I_{6}$ violates parity.

\subsubsection{Gravity as transfer $I_{2}$}

Information transfer $I_{2}$ with $R=S=A, T=B$ and $\tau_{0}=\tau_{A}<\tau_{B}=\tau_{E}$ is the only tensorbased and parity non-violating transfer which exerts a non-zero force and can work among all the particles in the Universe. Therefore, it is the only transfer which can represent gravity. Let us mention several of its properties.

Gravity, in order to work, does not require any entanglement, and thus is the only fully local information transfer. It means that gravity should define and also affect what being local really means. Gravity satisfies $\tau_{0}=\tau_{A}$, which means that the value of $\Delta q$, i.e., the value of momentum and other transferable conserved quantities that will be transferred to the final particle, is determined at the beginning of the transfer. This is the key difference from the other three fundamental interactions which all satisfy $\tau_{0}<\tau_{A}$. The value of $\Delta q$ remains constant and stored in mediator $M_{2}$ until the end of the transfer. The determination of $\triangle q$ at the very beginning of the transfer and keeping it unchanged to the very end makes gravity very classical, something we do not expect in genuine quantum gravity. However, there is no way to avoid this property in gravity, and denying it will always lead to constructing non-viable quantum gravity models. Thus, gravity can be described by quantum framework only when the equation of motion respects the constancy of $\Delta q$. Since $\tau_{0}=\tau_{A}<\tau_{B}=\tau_{E}$, mediator $M_{2}$ is always real and never virtual.

\subsection{Unification of fundamental interactions}

We distinguish three types of unification of the fundamental interactions: conceptual, mathematical and phenomenological unification. By the conceptual unification we mean that there exists a set of postulates which implies the existence of all the known fundamental interactions. Postulates 3 and 4 are such postulates, and therefore we claim that the conceptual unification has been achieved by this article.

By the mathematical unification we mean that there exists a single mathematical framework being able to fully describe all the fundamental interactions. Evidently, building such a framework is substantially easier when the conceptual unification is achieved. Although we have not expressed the full framework, we claim that its spatial part should be based on $Q_{i}(\mathbf{x}, \mathbf{q})$, a quantity which expresses dynamic differences among the information transfers and which gives rise to directional distribution $K_{i}(\mathbf{x}, \hat{\mathbf{e}})$. Our model predicts that electromagnetic, weak and strong interactions can be within that framework described identically only within time interval $\left(\tau_{0}, \tau_{A}\right)$ because at time $\tau_{0}$ they differ in the usage of entanglements, and at time $\tau_{A}$ each of them starts to behave in a different way. Gravity with $\tau_{0}=\tau_{A}$ has no time interval $\left(\tau_{0}, \tau_{A}\right)$, and thus it cannot be described in the way the other three fundamental interactions can be. 
It is widely believed that there exists a threshold point (such as a threshold energy) above which all the known fundamental interactions behave in the same way. This is what we mean by the phenomenological unification. Since two different information transfers use different timings and/or different role distributions, they are intrinsically different. Thus, we claim that the phenomenological unification may exist only when an extreme environment above the threshold point hides the differences between the transfers. In other words, the recovery of broken symmetries is nothing more than an environmental inability to demonstrate all the details of the transfers. Electromagnetic, weak and strong interaction may be unified phenomenologically when all $\tau_{A}-\tau_{0}$ of the corresponding information transfers become of the same order. Since gravity satisfies $\tau_{A}-\tau_{0}=0$, the phenomenological unification of all the four fundamental interactions is possible only when the threshold point is at the very limit of the system.

\section{Discussion}

We have shown a way to derive a model of the fundamental interactions and their basic physical properties from four simple postulates and one dynamic law. It required thinking computationally about physics, the complement to thinking physically about computation usually used in quantum computation and quantum information [49]. Namely, we have assumed our Universe is a computer system, have developed some analogies between physics and computer science, and have used computer science concepts to say how our Universe may work. We have started with a derivation of the list of the fundamental information transfers, that is the list of different ways information can be transferred from one particle to another. Then, for each non-instantaneous loopless information transfer we have found out what entanglements it requires, how it influences interacting point-like particles, and what basic symmetries it has. This made possible to identify what information transfers can represent gravitational scattering, electromagnetic scattering, strong scattering and weak decay (see the periodic table 3). Besides that, we have learnt several lessons about properties of the individual fundamental interactions. For example, we have seen that confinement in strong interaction can be derived from first principles (asked in $[50,51]$ ) and that parity violation in weak decay comes from the geometry of the interaction (asked in [52,53]). We have also seen that gravity fundamentally differs from the other three interactions in how it behaves at its initial point, and that this behavior brings an unexpected constraint on quantum gravity (asked in [54]). At a general level, we have learnt that the fundamental interactions are intrinsically different from each other. This fact substantially limits the phenomenological unification of the fundamental interactions at high energies. On the other hand, we have learnt that the fundamental interactions cannot have arbitrary properties-each interaction inherits its properties from the used information transfer(s). This implies that, in contrast to string theories [55,56], the presented model has a quite modest landscape, and thus indirectly limits several multiverse models [57].

The presented model can and definitely should be tested. Since it resides naturally in the low energy limit, many of its properties are testable with today's technologies. The key prediction of the model is the existence of entanglements in most of the fundamental interactions. We encourage this conceptual prediction to be tested because its experimental falsification would mean that the fundamental interactions of our Universe could not be described by the model presented in this study. The model would fail once and for all. For electromagnetic scattering and weak decay the model predicts an entanglement between the intermediate vector boson-antiboson pair and its source particle. For every two strongly interacting particles, 
it predicts that they strongly interact with each other only if they are entangled. Gravity is the only fundamental interaction which does not require any entanglement.

The presented model has the potential to solve some Standard Model puzzles and confront other models. Since this article is only its starting point, there are plenty of things which should be done in order to be used in that way: 1) We have not studied all the information transfers. Namely, we have not studied 4 instantaneous information transfers with $\tau_{0}=\tau_{E}$ and $S \neq T$, and 32 loop transfers with $S=T$. We have also not touched 8 self-transfers with only two participants: particle $A=B$ and mediator $M .2$ ) We have not identified in the model all the known processes, such as weak scattering, strong hadronization and Higgs mechanism, whether or not they are proper information transfers or an integral part of some transfer(s). Is the acquiring of mass related to the zero average forces $\left(I_{1}, I_{3}, I_{9}, I_{11}, I_{20}\right.$ and $\left.I_{28}\right)$ or even to any information transfer whose dispersion tensor has all diagonal elements non-zero? Finding the answer may shed light on mass hierarchy [58] and also even on the hadron mass spectrum [59]. If dark energy is an interaction and if it is independent of gravity (i.e., if it is not described by the cosmological constant model $[60,61]$ or any modified gravity model, such as $f(R)$ [62] and DGP model [63]), is it related to constant forces ( $I_{17}$ to $I_{19}$ and $I_{25}$ to $\left.I_{27}\right)$ ? We can also ask about the not mapped information transfers. Transfers $I_{6}$ and $I_{14}$ are the universal and the non-universal version of weak decay, respectively. They differ only in the particle that plays the role of particle $R$. Does it mean that transfer $I_{10}$ is a non-universal gravity, and that $I_{12}$ is a non-universal electromagnetic interaction (e.g., playing a role in the fractional electric charge of quarks)? 3) We have not identified how electric charge, spin, baryon number, mass and other important physical quantities appear in the model. Similarly, we have not derived the set of elementary particles that the model implies. Nevertheless, the variants of the individual information transfers (caused by $s_{q}$ and $s_{v}$ ) together with grouping the transfers with the same requirements (such as the existence of $\varepsilon(R, \bar{R})$ ) seem to be a plausible way to do that. Finding the set of elementary particles that the model implies may shed light on particle generation problem [16] and help to set a limit on technicolor [64,65] and see-saw mechanism models [66]. 4) We have not expressed a relation between quantum field theory and the presented model. If we limit ourselves only to perturbation regime of quantum field theory, this can be done by finding a relation between Feynman diagrams and $q$-diagrams. Since the derivation of asymptotic freedom in Yang-Mills theories requires a series of Feynman diagrams [67] but only one $q$-diagram, $q$-diagrams seem to be a very efficient way to describe processes. 5) We have not developed fully relativistic quantum model. Nevertheless, we have seen that the model already contains several quantum and relativistic properties without even postulating quantum theory and special relativity. Since we are convinced that every viable theory of all the fundamental interactions must imply quantum theory and relativity theory, at least as limit cases, we should first find out whether they can be fully derived from the presented model rather than impose them via an explicit postulation. 6) We have not done any detailed analysis of the fundamental information transfers. For example, we have seen that the dispersion tensor of gravity tends to disperse particles in the maximal way. Thus, once we leave out the assumption of point-like particles, the dispersion may be responsible for the weakening of gravitational force even in 3D space. Does it explain gauge hierarchy problem [58] without the necessity of extra-dimensional models [68-70] and string models [71]? Similarly, we have seen that despite the fact that weak decay and strong interaction satisfy the same condition $\tau_{0}<\tau_{A}<\tau_{E}$, only strong interaction transfers conserved quantities to particle $B$ always via entanglements, that is instantaneously over an arbitrary distance. Can this behavior resolve strong CP problem [72-74] and/or tell us why we have not observed axions so far [75]? Finally, we have seen that the model contains many information transfers. Can any of them explain the recent measurements of the anomalous magnetic dipole moment 
of the muon [76,77], or is a more complex mechanism needed? 7) We have not asked many questions which could not be answered before the model has been presented. For example: Do there exist new interactions we have not observed yet? And most importantly, if so, what are their properties? We have seen that in our Universe exist various information transfers. However, we do not see any reason why our Universe would use only a few information transfers (i.e., What would prevent our Universe from using the other transfers?) and why it would be exactly that particular combination of transfers, no matter what the combination is. Therefore, in agreement with postulates 3 and 4, we propose that every fundamental information transfer is demonstrated in our Universe. Theoretical studies of the transfers should reveal their properties in detail and make possible to build ingenious experiments capable of testing that proposition. Everything is substantially easier when we know exactly what we are looking for.

Acknowledgements We would like to thank Pavel Roušar for interesting and useful discussions on the early draft of this article, Luis Ochoa Torcal for continual encouragement and Ladislav Kišš for the linguistic revision of the text.

Author contributions The author declares that he is the only author of this article.

Funding The creation of this article has not been supported by any individual or any institute.

Availability of data and material Not applicable, this article contains a theoretical research.

\section{Declarations}

Conflicts of interest/Competing interests The author declares no conflict of interest.

Code availability Not applicable, no software has been used.

Open Access This article is licensed under a Creative Commons Attribution 4.0 International License, which permits use, sharing, adaptation, distribution and reproduction in any medium or format, as long as you give appropriate credit to the original author(s) and the source, provide a link to the Creative Commons licence, and indicate if changes were made. The images or other third party material in this article are included in the article's Creative Commons licence, unless indicated otherwise in a credit line to the material. If material is not included in the article's Creative Commons licence and your intended use is not permitted by statutory regulation or exceeds the permitted use, you will need to obtain permission directly from the copyright holder. To view a copy of this licence, visit http://creativecommons.org/licenses/by/4.0/.

\section{References}

1. S. Weinberg, Dreams of Final Theory (Vintage book, UK, 1992)

2. S.L. Glashow, Partial-symmetries of weak interactions. Nucl. Phys. 22(4), 579-588 (1961). https://doi. org/10.1016/0029-558228612990469-2

3. S. Weinberg, A model of leptons. Phys. Rev. Lett. 19(21), 1264-1266 (1967). https://doi.org/10.1103/ PhysRevLett.19.1264

4. A. Salam, J.C. Ward, Electromagnetic and weak interactions. Phys. Lett. 13(2), 168-171 (1964). https:// doi.org/10.1016/0031-916328642990711-5

5. A. Salam, N. Svartholm, "Elementary particle theory," Ed. N. Svartholm, Stockholm, Almquist and Wiksell, vol. 367, (1968)

6. H. Georgi, S.L. Glashow, Unity of all elementary-particle forces. Phys. Rev. Lett. 32(8), 438-441 (1974). https://doi.org/10.1103/PhysRevLett.32.438

7. H. Georgi, H.C. Wolfe, C.E. Carlson, "The state of the art-gauge theories," In AIP Conf. Proc., vol. 23, pp. 575-582, AIP, (1975). https://doi.org/10.1063/1.2947450

8. J.C. Pati, A. Salam, Lepton number as the fourth “color". Phys. Rev. D 10(1), 275-289 (1974). https:// doi.org/10.1142/97898127959155f0042 
9. G. Senjanović, "Proton decay and grand unification," In AIP Conf. Proc., vol. 1200, pp. 131-141, AIP, (2010). https://doi.org/10.1063/1.3327552, arXiv:0912.5375 [hep-ph]

10. C. Patrignani et al., Review of particle physics. Chin. Phys. C 40(10), 100001 (2016). https://doi.org/10. 1088/1674-1137/40/10/100001

11. R..N. Mohapatra, Unification and Supersymmetry: The Frontiers of Quark-Lepton Physics (Springer, New York, 2003)

12. P. Nath, Supersymmetry, supergravity, and unification (Cambridge University Press, Cambridge, 2016)

13. A. Cakir, "Searches for supersymmetry with the CMS experiment." https://doi.org/10.1142/ 97898144368305f0011, arXiv:1111.4820 [hep-ph], (2011)

14. X.P. Bueso, "Supersymmetry searches at the Tevatron and the LHC collider experiments." arXiv:1112.1723 [hep-ex], (2011)

15. A. collaboration et al., "Summary of the ATLAS experiment's sensitivity to supersymmetry after LHC run 1-interpreted in the phenomenological MSSM." https://doi.org/10.1007/JHEP1028201529134, arXiv:1508.06608 [hep-ex], (2015)

16. Y. Nagashima, Elementary Particle Physics: Quantum field theory and particles, vol. 1 (Wiley, Hoboken, 2011)

17. J.D. Barrow, New theories of everything: the quest for ultimate explanation (Oxford University Press, UK, 2008)

18. J. Cham, D. Whiteson, We have no idea: A guide to the unknown universe (Penguin, UK, 2017)

19. K. Zuse, "Rechnender raum," Elektronische Datenverarbeitung, vol. 8, pp. 336-344, 1967. English translation at ftp://ftp.idsia.ch/pub/juergen/zuserechnenderraum.pdf

20. J.A. Wheeler, The computer and the universe. Int. J. Theor. Phys. 21(6), 557-572 (1982). https://doi.org/ 10.1007/BF02650185

21. S. Wolfram, A New Kind of Science (Wolfram Media, Illinois, 2002)

22. G. Fontana, "Why we live in the computational universe." arXiv:physics/0511157, excerpt from https:// doi.org/10.1063/1.2169325, (2005)

23. S. Lloyd, Programming the Universe: A Quantum Computer Scientist Takes on the Cosmos (Vintage Books, New York, 2006)

24. S. Lloyd, "The universe as quantum computer," A Computable Universe: Understanding and exploring Nature as computation, pp. 567-581, (2013). https://doi.org/10.1142/97898143743095f0029, also at arXiv: 1312.4455 [quant-ph]

25. D.R. Finkelstein, "Nature as quantum computer," in From Linear Operators to Computational Biology, pp. 5-22, Springer, (2013). https://doi.org/10.1007/978-1-4471-4282-95f2, also at arXiv:1201.1599 [quant-ph]

26. S. Gudder, The universe and the quantum computer. Int. J. Theor. Phys. 50(12), 3800-3811 (2011). https:// doi.org/10.1007/s10773-011-0667-4. arXiv:1405.0638 [gr-qc]

27. P. Gibbs, "A universe programmed with strings of qubits." arXiv:1106.3029 [hep-th], (2011)

28. J. Schmidhuber, "A computer scientist's view of life, the universe, and everything," In Foundations of Computer Science, pp. 201-208, Springer, (1997). also at arXiv:quant-ph/9904050

29. D. Deutsch, "Physics, philosophy and quantum technology," In Proceedings of the Sixth International Conference on Quantum Communication, Measurement and Computing, pp. 419-426, (2003)

30. F. Markopoulou, “The computing spacetime," In How the World Computes, pp. 472-484, Springer, (2012). https://doi.org/10.1007/978-3-642-30870-35f48, also at arXiv:1201.3398 [gr-qc]

31. J.A. Wheeler, "Information, Physics, Quantum: The Search for Links," In Proc 3rd Int (Symp. Foundation of Quantum Mechanics, Tokyo, 1989), pp. 354-368

32. J. Paquet, S.A. Mokhov, "Comparative studies of programming languages; course lecture notes." arXiv:1007.2123 [cs.PL], (2010)

33. F. Markopoulou, "Space does not exist, so time can." arXiv:0909.1861 [gr-qc], (2009)

34. C. Neipp, A. Hernández, J. Rodes, A. Márquez, T. Beléndez, A. Beléndez, An analysis of the classical Doppler effect. Eur. J. Phys. 24(5), 497-505 (2003)

35. D.J. Griffiths, Introduction to Electrodynamics, 4th editon (Cambridge University Press, UK, 2017)

36. R. Aaij, B. Adeva, M. Adinolfi, A. Affolder, Z. Ajaltouni, J. Albrecht, F. Alessio, M. Alexander, S. Ali, G. Alkhazov et al., Observation of the resonant character of the Z(4430) ${ }^{-}$state. Phys. Rev. Lett. 112(22), 222002 (2014). https://doi.org/10.1103/PhysRevLett.112.222002

37. A. Badalov, L. Garrido Beltrán, D. Gascón Fora, R. Graciani Díaz, C. Marin Benito, E. Picatoste Olloqui, V.J. Rives Molina, X. Vilasis-Cardona, LHCb Collaboration, et al., "Observation of $J / \psi \phi$ structures consistent with exotic states from amplitude analysis of $B^{+} \rightarrow J / \psi \phi K^{+}$decays," Phys. Rev. Lett., vol. 118, p.022003, (2017). https://doi.org/10.1103/PhysRevLett.118.022003, also at arXiv:1606.07895 [hep-ex] 
38. R. Aaij, B. Adeva, M. Adinolfi, Z. Ajaltouni, S. Akar, J. Albrecht, F. Alessio, M. Alexander, S. Ali, G. Alkhazov, et al., "Amplitude analysis of $B^{+} \rightarrow J / \psi \phi K^{+}$decays." arXiv:1606.07898 [hep-ex], (2016)

39. R. Aaij, B. Adeva, M. Adinolfi, A. Affolder, Z. Ajaltouni, S. Akar, J. Albrecht, F. Alessio, M. Alexander, S. Ali et al., Observation of $J / \psi p$ resonances consistent with pentaquark states in $\Lambda_{b}^{0} \rightarrow J / \psi K^{-} p$ decays. Phys. Rev. Lett. 115(7), 072001 (2015). https://doi.org/10.1103/PhysRevLett.115.072001

40. S. Bethke, Experimental tests of asymptotic freedom. Nucl. Phys. B - Proceedings Supplements 54(1-2), 314-324 (1997). https://doi.org/10.1016/S0920-563228972900061-3. arXiv:hep-ex/9609014

41. K.G. Wilson, Confinement of quarks. Phys. Rev. D 10(8), 2445 (1974)

42. E. Eichten, K. Gottfried, T. Kinoshita, K. Lane, T.-M. Yan, Charmonium: the model. Phys. Rev. D 17(11), 3090 (1978). https://doi.org/10.1103/PhysRevD.17.3090

43. C.-S. Wu, E. Ambler, R. Hayward, D. Hoppes, R.P. Hudson, Experimental test of parity conservation in beta decay. Phys. Rev. 105(4), 1413 (1957). https://doi.org/10.1103/PhysRev.105.1413

44. J. Hořejší, Fundamentals of Electroweak Theory (Karolinum Press, Czech Republic, 2002)

45. T.-D. Lee, C.-N. Yang, Question of parity conservation in weak interactions. Phys. Rev. 104(1), 254 (1956). https://doi.org/10.1103/PhysRev.104.254

46. F. Koks, J. Van Klinken, Investigation on $\beta$-polarization at low velocities with $\beta$-particles from the decay of tritium. Nucl. Phys. A 272(1), 61-81 (1976). https://doi.org/10.1016/0375-947428762990318-3

47. E.D. Commins, P.H. Bucksbaum, Weak interactions of leptons and quarks (Cambridge University Press, UK, 1983)

48. N.M.J. Woodhouse, General Relativity (Springer, New York, 2007)

49. M.A. Nielsen, I.L. Chuang, Quantum Computation and Quantum Information (Cambridge University Press, UK, 2009)

50. A.V. Smilga, Lectures on Quantum Chromodynamics (World Scientific, Singapore, 2001)

51. V. Christianto, F. Smarandache, "Thirty Unsolved Problems in the Physics of Elementary Particles," Neutrosophic Logic, Wave Mechanics, and other stories, pp. 26-28, (2009)

52. B. Desplanques, "Parity violation and the nature of charges," In Proceedings of The 3rd Workshop From Parity Violation to Hadronic Structure and more..., pp. 207-209, Springer, (2007). https://doi.org/10. 1007/978-3-540-74413-95f37, also at arXiv:hep-ph/0612040

53. C.D. Froggatt, H.B. Nielsen, "Why do we have parity violation?." arXiv:hep-ph/9906466, (1999)

54. L. Smolin, "What are we missing in our search for quantum gravity?," In Foundations of Mathematics and Physics One Century After Hilbert, pp. 287-304, Springer, (2018). https://doi.org/10.1007/978-3319-64813-25f11, also at arXiv:1705.09208 [gr-qc]

55. F. Denef, M.R. Douglas, Distributions of flux vacua. J. High Ene. Phys. 2004(05), 072 (2004). https:// doi.org/10.1088/1126-6708/2004/05/072

56. W. Taylor, Y.-N. Wang, The F-theory geometry with most flux vacua. J. High Ene. Phys. 2015(12), 1-21 (2015). https://doi.org/10.1007/JHEP1228201529164

57. B. Greene, The hidden reality: Parallel universes and the deep laws of the cosmos (Vintage, New York, 2011)

58. Y. Nagashima, Beyond the Standard Model of Elementary Particle Physics (Wiley, Hoboken, 2014)

59. A. Majumder, B. Müller, Hadron mass spectrum from lattice QCD. Phys. Rev. Lett. 105(25), 252002 (2010). https://doi.org/10.1103/PhysRevLett.105.252002. arXiv:1008.1747 [hep-ph]

60. L. Amendola, S. Tsujikawa, Dark energy: Theory and Observations (Cambridge University Press, Cambridge, 2010)

61. Y. Wang, Dark energy (Wiley, Hoboken, 2009)

62. R.R. Caldwell, M. Kamionkowski, The physics of cosmic acceleration. Ann. Rev. Nuc. Part. Sci. 59, 397-429 (2009). https://doi.org/10.1146/annurev-nucl-010709-151330

63. G. Dvali, G. Gabadadze, M. Porrati, 4D gravity on a brane in 5D Minkowski space. Phys. Lett. B 485(1-3), 208-214 (2000). https://doi.org/10.1016/S0370-269328002900669-9 arXiv:hep-th/0005016

64. S. Dimopoulos, L. Susskind, Mass without scalars. Nucl. Phys. B 155(1), 237-252 (1979)

65. E. Eichten, K. Lane, Dynamical breaking of weak interaction symmetries. Phys. Lett. B 90(1-2), 125-130 (1980). https://doi.org/10.1016/0370-269328802990065-9

66. J.W.F. Valle, J. Romão, Neutrinos in High energy and Astroparticle Physics (Wiley, Hoboken, 2015)

67. D.J. Gross, F. Wilczek, Ultraviolet behavior of non-Abelian gauge theories. Phys. Rev. Lett. 30(26), 1343 (1973). https://doi.org/10.1103/PhysRevLett.30.1343

68. N. Arkani-Hamed, S. Dimopoulos, G. Dvali, Phenomenology, astrophysics, and cosmology of theories with submillimeter dimensions and TeV scale quantum gravity. Phys. Rev. D 59(8), 086004 (1999). https://doi.org/10.1103/PhysRevD.59.086004. arXiv:hep-ph/9807344

69. N. Arkani-Hamed, S. Dimopoulos, G. Dvali, The hierarchy problem and new dimensions at a millimeter. Phys. Lett. B 429(3-4), 263-272 (1998). https://doi.org/10.1016/S0370-269328982900466-3 arXiv:hep-ph/9803315 
70. L. Randall, R. Sundrum, Large mass hierarchy from a small extra dimension. Phys. Rev. Lett. 83(17), 3370 (1999). https://doi.org/10.1103/PhysRevLett.83.3370. arXiv:hep-ph/9905221

71. M.B. Green, J.H. Schwarz, Supersymmetrical string theories. Phys. Lett. B 109(6), 444-448 (1982). https://doi.org/10.1016/0370-269328822991110-8

72. G.C. Branco, L. Lavoura, J.P. Silva, CP violation, vol. 103 (Oxford University Press, UK, 1999)

73. R.D. Peccei, H.R. Quinn, CP conservation in the presence of pseudoparticles. Phys. Rev. Lett. 38(25), 1440 (1977). https://doi.org/10.1103/PhysRevLett.38.1440

74. R.D. Peccei, “The strong CP problem,” In CP violation, pp. 501-551, World Scientific, (1989)

75. R. Battesti, B. Beltrán, H. Davoudiasl, M. Kuster, P. Pugnat, R. Rabadan, A. Ringwald, N. Spooner, K. Zioutas, "Axion searches in the past, at present, and in the near future," In Axions, pp. 199-237, Springer, 2008. https://doi.org/10.1007/978-3-540-73518-25f10, also at arXiv:0705.0615 [hep-ex]

76. B.L. Roberts, W.J. Marciano, Lepton dipole moments, vol. 20 (World Scientific, Singapore, 2010)

77. B. Abi, T. Albahri, S. Al-Kilani, D. Allspach, L. Alonzi, A. Anastasi, A. Anisenkov, F. Azfar, K. Badgley, S. Baeßler et al., Measurement of the positive muon anomalous magnetic moment to $0.46 \mathrm{ppm}$. Phys. Rev. Lett. 126(14), 141801 (2021). https://doi.org/10.1103/PhysRevLett.126.141801 